\title{
Abstractions for Security Protocol Verification
}

\section{Report}

Author(s):

Nguyen, Thanh Binh; Sprenger, Christoph

Publication date:

2015

Permanent link:

https://doi.org/10.3929/ethz-a-010347780

Rights / license:

In Copyright - Non-Commercial Use Permitted

\section{Funding acknowledgement:}

256980 - Network of Excellence on Engineering Secure Future Internet Software Services and Systems (EC) 


\title{
Abstractions for Security Protocol Verification
}

\author{
Binh Thanh Nguyen and Christoph Sprenger \\ Institute of Information Security \\ Department of Computer Science, ETH Zurich, Switzerland
}

\begin{abstract}
We present a large class of security protocol abstractions with the aim of improving the scope and efficiency of verification tools. We propose typed abstractions, which transform a term's structure based on its type, and untyped abstractions, which remove atomic messages, variables, and redundant terms. Our theory improves on previous work by supporting a useful subclass of shallow subterm-convergent rewrite theories, user-defined types, and untyped variables to cover type flaw attacks. We prove soundness results for an expressive property language that includes secrecy and authentication. Applying our abstractions to realistic IETF protocol models, we achieve dramatic speedups and extend the scope of several modern security protocol analyzers.
\end{abstract}

\section{Introduction}

Security protocols play a central role in today's networked applications. Past experience has amply shown that informal arguments justifying the security of such protocols are insufficient. This makes security protocols prime candidates for formal verification. In the last two decades, research in formal security protocol verification has made enormous progress, which is reflected in many state-of-the-art tools including AVANTSSAR [1], ProVerif [6], Maude-NPA [14], Scyther [10], and Tamarin [21]. These tools can verify small to medium-sized protocols in a few seconds or less, sometimes for an unbounded number of sessions. Despite this success, they can still be challenged when verifying real-world protocols such as those defined in standards and deployed on the internet (e.g., TLS, IKE, and ISO/IEC 9798). Such protocols typically have messages with numerous fields, support many alternatives (e.g., cryptographic setups), and may be composed from more basic protocols (e.g., IKEv2-EAP).

Abstraction [7] is a standard technique to over-approximate complex systems by simpler ones for verification. Sound abstractions preserve counterexamples (or attacks in security terms) from concrete to abstracted systems. In the context of security protocols, abstractions are extensively used. Here, we only mention a few examples. First, the Dolev-Yao model is a standard (not necessarily sound) abstraction of cryptography. Second, many tools use abstractions to map the verification problem into the formalism of an efficient solver or reasoner. We call these back-end abstractions. For example, ProVerif [6] translates models in the applied pi calculus to a set of Horn clauses, SATMC [4] reduces protocol verification to SAT solving, and Paulson [23] models protocols as inductively 
defined trace sets. Finally, some abstractions aim at speeding up automated analysis by simplifying protocols within a given protocol model before feeding them to verifiers $[18,22]$. Our work belongs to this class of front-end abstractions.

Extending Hui and Lowe's work [18], we proposed in [22] a rich class of protocol abstractions and proved its soundness for a wide range of security properties. We used a type system to uniformly transform all terms of a given type (e.g., a pattern in a protocol role and its instances during execution) whereas [18] only covers ground terms. Our work [22] exhibits several limitations: (1) the theory is limited to the free algebra over a fixed signature; (2) all variables have strict (possibly structured) types, hence we cannot precisely model ticket forwarding or Diffie-Hellman exchanges. While the type system enables fine-grained control over abstractions (e.g., by discerning different nonces), it may eliminate realistic attacks such as type flaw attacks; (3) some soundness conditions involving quantifiers are hard to check in practice; and (4) it presents few experimental results for a single tool (SATMC) using abstractions that are crafted manually.

In this work, we address all the limitations above. First, we work with a useful subclass of shallow subterm-convergent rewrite theories modulo a set of axioms to model cryptographic operations. Second, we support untyped variables, userdefined types, and subtyping. User-defined types enable the grouping of similar atomic types (e.g., session keys) and adjusting the granularity of matching in message abstraction. Third, we have separated the removal of variables, atomic messages, and redundancies (new untyped abstractions) from the transformation of the message structure (typed abstractions). This simplifies the specifications and soundness proof of typed abstractions. Fourth, we provide effectively checkable syntactic criteria for the conditions of the soundness theorem. Finally, we extended Scyther [10] with fully automated support for our abstraction methodology. We validated our approach on an extensive set of realistic case studies drawn from the IKEv1, IKEv2, ISO/IEC 9798, and PANA-AKA standard proposals. Our abstractions result in very substantial performance gains. We have also obtained positive results for several other state-of-the-art verifiers (ProVerif, CL-Atse, OFMC, and SATMC) with manually produced abstractions.

Example: The IKEv2-mac protocol The Internet Key Exchange (IKE) family of protocols is part of the IPsec protocol suite for securing Internet Protocol (IP) communication. IKE establishes a shared key, which is later used for securing IP packets, realizes mutual authentication, and offers identity protection as an option. Its first version (IKEv1) dates back to 1998 [17]. The second version (IKEv2) [20] significantly simplifies the first one. However, the protocols in this family are still complex and contain a large number of fields.

Concrete protocol. As our running example, we present a member of the IKEv2 family, called IKEv2-mac (or IKE $\mathrm{m}_{\mathrm{m}}$ for short), which sets up a session key using a Diffie-Hellman (DH) key exchange, provides mutual authentication based on MACs, and also offers identity protection. We use Cremers' models of IKE [11] as a basis for our presentation and experiments (see Section 4.2). Our starting point 
is the following concrete $\mathrm{IKE}_{\mathrm{m}}$ protocol between an initiator $A$ and a responder $B$.

$$
\begin{array}{ll}
\operatorname{IKE}_{\mathrm{m}}(1) . & A \rightarrow B: S P I a, o, s A 1, g^{x}, N a \\
\operatorname{IKE}_{\mathrm{m}}(2) . & B \rightarrow A: S P I a, S P I b, s A 1, g^{y}, N b \\
\operatorname{IKE}_{\mathrm{m}}(3) . & A \rightarrow B: S P I a, S P I b,\{|A, B, A U T H a, s A 2, t S a, t S b|\}_{S K} \\
\operatorname{IKE}_{\mathrm{m}}(4) . & B \rightarrow A: S P I a, S P I b,\{|B, A U T H b, s A 2, t S a, t S b|\}_{S K}
\end{array}
$$

Here, SPIa and SPIb denote the Security Parameter Indices that determine cryptographic algorithms, $o$ is a constant number, $s A 1$ and $s A 2$ are Security Associations, $g$ is the DH group generator, $x$ and $y$ are secret $\mathrm{DH}$ exponents, $\mathrm{Na}$ and $N b$ are nonces, and $t S a$ and $t S b$ denote Traffic Selectors specifying certain IP parameters. $A U T H a$ and $A U T H b$ denote the authenticators of $A$ and $B$ and $S K$ the session key derived from the DH key $g^{x y}$. These are defined as follows.

$$
\begin{aligned}
S K & =\operatorname{kdf}\left(N a, N b, g^{x y}, S P I a, S P I b\right) \\
A U T H a & =\operatorname{mac}\left(\operatorname{sh}(A, B), S P I a, o, s A 1, g^{x}, N a, N b, \operatorname{prf}(S K, A)\right) \\
A U T H b & =\operatorname{mac}\left(\operatorname{sh}(B, A), S P I a, S P I b, s A 1, g^{y}, N b, N a, \operatorname{prf}(S K, B)\right)
\end{aligned}
$$

We model the functions mac, kdf, and prf as hash functions and use $\operatorname{sh}(A, B)$ and $\operatorname{sh}(B, A)$ to refer to the (single) long-term symmetric key shared by $A$ and $B$.

We consider the following security properties: (P1) the secrecy of the DH key $g^{x y}$, which implies the secrecy of $S K$, and (P2) mutual non-injective agreement on the nonces $N a$ and $N b$ and the DH half-keys $g^{x}$ and $g^{y}$.

Abstraction. Our theory supports the construction of abstract models by removing inessential fields and operations. For example, in $\mathrm{IKE}_{\mathrm{m}}$ we can remove: (i) the symmetric encryptions with the session key $S K$; then (ii) all atomic top-level fields except $N a$ and $N b$; (iii) all fields of $S K$ except the DH key $g^{x y}$; and (iv) from the authenticators: the fields $S P I a, S P I b$, and $s A 1$ and the application of prf including the agent names underneath. The resulting protocol is $\mathrm{IKE}_{\mathrm{m}}^{2}$ :

$$
\begin{aligned}
& \mathrm{IKE}_{\mathrm{m}}^{2}(1) . A \rightarrow B: g^{x}, N a \quad \operatorname{IKE}_{\mathrm{m}}^{2}(3) . A \rightarrow B: A U T H a \\
& \mathrm{IKE}_{\mathrm{m}}^{2}(2) . B \rightarrow A: g^{y}, N b \quad \mathrm{IKE}_{\mathrm{m}}^{2}(4) . B \rightarrow A: A U T H b
\end{aligned}
$$

where $S K=\operatorname{kdf}\left(g^{x y}\right)$ and $A U T H a=\operatorname{mac}\left(\operatorname{sh}(A, B), o, g^{x}, N a, N b, S K\right)$ for role $A$ and $A U T H b=\operatorname{mac}\left(\operatorname{sh}(B, A), g^{y}, N b, N a, S K\right)$ for role $B$.

Scyther verifies the properties $(\mathrm{P} 1)$ and $(\mathrm{P} 2)$ in $8.7 \mathrm{~s}$ on the concrete and in $1.7 \mathrm{~s}$ on an automatically generated abstract protocol (which is less intuitive than the one presented here). Our soundness results imply that the original protocol $\mathrm{IKE}_{\mathrm{m}}$ also enjoys these properties. We chose the protocol IKE $\mathrm{K}_{\mathrm{m}}$ as running example for its relative simplicity compared to the other protocols in our case studies. In many of our experiments (Section 4.2), our abstractions (i) result in much more substantial speedups, or (ii) enable the successful unbounded verification of a protocol where it times out or exhausts memory on the original protocol.

\section{Security protocol model}

We define a term algebra $\mathcal{T}_{\Sigma}(V)$ over a signature $\Sigma$ and a set of variables $V$ in the standard way. Let $\Sigma^{n}$ denote the symbols of arity $n$. We call the elements of $\Sigma^{0}$ 
atoms and write $\Sigma^{\geq 1}$ for the set of proper function symbols. For a fixed $\Sigma^{\geq 1}$, we will vary $\Sigma^{0}$ to generate different sets of terms, denoted by $\mathcal{T}\left(V, \Sigma^{0}\right)$, including terms in protocol roles, network messages, and types. We write $s u b s(t)$ for the set of subterms of $t$ and define the size of $t$ by $|t|=|\operatorname{subs}(t)|$. We also define $\operatorname{vars}(t)=$ $\operatorname{subs}(t) \cap V$. If $\operatorname{vars}(t)=\emptyset$ then $t$ is called ground. We denote the top-level symbol of a (non-variable) term $t$ by top $(t)$ and the set of its symbols in $\Sigma^{\geq 1}$ by $c t(t)$. A position is a sequence of natural numbers. We denote the subterm of $t$ at position $p$ with $\left.t\right|_{p}$ and write $t[u]_{p}$ for the term obtained by replacing $\left.t\right|_{p}$ at position $p$ by $u$. We also partition $\Sigma$ into sets of public and private symbols, denoted by $\Sigma_{\text {pub }}$ and $\Sigma_{\text {pri }}$. We assume $\Sigma_{\text {pub }}$ includes pairing $\langle\cdot, \cdot\rangle$ which associates to the right, e.g., $\langle t, u, v\rangle=\langle t,\langle u, v\rangle\rangle$. We usually write, e.g., $\{|t, u, v|\}_{k}$ rather than $\{\langle t, u, v\rangle \mid\}_{k}$. We define the splitting function by $\operatorname{split}(\langle t, u\rangle)=\operatorname{split}(t) \cup \operatorname{split}(u)$ on pairs and split $(t)=\{t\}$ on other terms $t$. We call the elements of $\operatorname{split}(t)$ the fields of $t$. For $n \in \mathbb{N}, \widetilde{n}$ denotes $\{1, \ldots, n\}$.

The set of message terms is $\mathcal{M}=\mathcal{T}(\mathcal{V}, \mathcal{A} \cup \mathcal{F} \cup \mathcal{C})$, where $\mathcal{V}, \mathcal{A}, \mathcal{F}$, and $\mathcal{C}$ are pairwise disjoint infinite sets of variables, agents, fresh values, and constants.

\subsection{Type system}

We introduce a type system akin to [2] and extend it with subtyping. We define the set of atomic types by $\mathcal{Y}_{a t}=\mathcal{Y}_{0} \cup\{\alpha, m s g\} \cup\left\{\beta_{n} \mid n \in \mathcal{F}\right\} \cup\left\{\gamma_{c} \mid c \in \mathcal{C}\right\}$, where $\alpha, \beta_{n}$, and $\gamma_{c}$ are the types of agents, the fresh value $n$, and the constant $c$, respectively. Moreover, $m s g$ is the type of all messages and $\mathcal{Y}_{0}$ is a disjoint set of user-defined types. The set of all types is then defined by $\mathcal{Y}=\mathcal{T}\left(\emptyset, \mathcal{Y}_{a t}\right)$.

We assume that all variables have an atomic type, i.e., $\mathcal{V}=\left\{\mathcal{V}_{\tau}\right\}_{\tau \in \mathcal{Y}_{a t}}$ is a family of disjoint infinite sets of variables. Let $\Gamma: \mathcal{V} \rightarrow \mathcal{Y}_{a t}$ be such that $\Gamma(X)=\tau$ if and only if $X \in \mathcal{V}_{\tau}$. We extend $\Gamma$ to atoms by defining $\Gamma(a)=\alpha, \Gamma(n)=\beta_{n}$, and $\Gamma(c)=\gamma_{c}$ for $a \in \mathcal{A}, n \in \mathcal{F}$, and $c \in \mathcal{C}$, and then homomorphically to all terms $t \in \mathcal{M}$. We call $\tau=\Gamma(t)$ the type of $t$ and sometimes also write $t: \tau$.

The subtyping relation $\preccurlyeq$ on types is defined by the following inference rules and by two additional rules (not shown) defining its reflexivity and transitivity.

$$
\frac{\tau \in \mathcal{Y}}{\tau \preccurlyeq m s g} \mathrm{~S}(m s g) \quad \frac{\tau_{1} \preccurlyeq 0 \tau_{2}}{\tau_{1} \preccurlyeq \tau_{2}} \mathrm{~S}(\preccurlyeq 0) \quad \frac{\tau_{1} \preccurlyeq \tau_{1}^{\prime} \cdots \tau_{n} \preccurlyeq \tau_{n}^{\prime}}{c\left(\tau_{1}, \ldots, \tau_{n}\right) \preccurlyeq c\left(\tau_{1}^{\prime}, \ldots, \tau_{n}^{\prime}\right)} \mathrm{S}\left(c \in \Sigma^{n}\right)
$$

Every type is a subtype of $m s g$ by the first rule. The second rule embeds a user-defined atomic subtyping relation $\preccurlyeq_{0} \subseteq\left(\mathcal{Y}_{a t} \backslash\{m s g\}\right) \times \mathcal{Y}_{0}$, which relates atomic types (except $m s g$ ) to user-defined atomic types in $\mathcal{Y}_{0}$. For simplicity, we require that $\preccurlyeq_{0}$ is a partial function. The third rule ensures that subtyping is preserved by all symbols. The set of subtypes of $\tau$ is $\tau \downarrow=\left\{\tau^{\prime} \in \mathcal{Y} \mid \tau^{\prime} \preccurlyeq \tau\right\}$.

\subsection{Equational theories}

An equation over a signature $\Sigma$ is an unordered pair $\{s, t\}$, written $s \simeq t$, where $s, t \in \mathcal{T}_{\Sigma}\left(\mathcal{V}_{\text {msg }}\right)$. An equation presentation $\mathcal{E}=(\Sigma, E)$ consists of a signature $\Sigma$ and a set $E$ of equations over $\Sigma$. The equational theory induced by $\mathcal{E}$ is the 
smallest $\Sigma$-congruence, written $={ }_{E}$, containing all instances of equations in $E$. We often identify $\mathcal{E}$ with the induced equational theory.

A rewrite rule is an oriented pair $l \rightarrow r$, where $\operatorname{vars}(r) \subseteq \operatorname{vars}(l) \subseteq \mathcal{V}_{m s g}$. A rewrite theory is a triple $\mathcal{R}=(\Sigma, A x, R)$ where $\Sigma$ is a signature, $A x$ a set of $\Sigma$-equations, and $R$ a set of rewrite rules. The rewriting relation $\rightarrow_{R, A x}$ on $\mathcal{T}_{\Sigma}(V)$ is defined by $t \rightarrow_{R, A x} t^{\prime}$ iff there exists a non-variable position $p$ in $t$, a rule $l \rightarrow r \in R$, and a substitution $\sigma$ such that $\left.t\right|_{p}={ }_{A x} l \sigma$ and $t^{\prime}=t[r \sigma]_{p}$. If $t \rightarrow_{R, A x}^{*} t^{\prime}$ and $t^{\prime}$ is irreducible, we call $t^{\prime} R, A x$-normal and also say that $t^{\prime}$ is a normal form of $t$. Under suitable termination, confluence, and coherence conditions (see [19] for definitions), one can decompose an equational theory $(\Sigma, E)$ into a rewrite theory $(\Sigma, A x, R)$ where $A x \subseteq E$ and, for all terms $t, u \in \mathcal{T}_{\Sigma}(V)$, we have $t={ }_{E} u$ iff $t \downarrow_{R, A x}={ }_{A x} u \downarrow_{R, A x}$. Here, $t \downarrow_{R, A x}$ denotes any normal form of $t$. In this paper, we work with decomposable equational theories.

A rewriting theory $R$ is subterm-convergent if it is convergent and, for each $l \rightarrow r \in R, r$ is either a proper subterm of $l$ or ground and in normal form with respect to $R$. For our soundness result, we consider the subclass $\mathcal{S}$ of subtermconvergent rewrite theories where each rule in $R$ has one of the following forms.

- (R1): $d\left(c\left(x_{1}, \ldots, x_{n}, t\right), u\right) \rightarrow x_{j}$, where $c, d \in \Sigma_{\text {pub }}, t, u$ are terms, $j \in \tilde{n}$, and $x_{1}, \ldots, x_{n}$ are pairwise distinct variables with $x_{i} \notin \operatorname{vars}(t, u)$ for all $i \in \tilde{n}$.

- (R2): $d\left(c\left(x_{1}, \ldots, x_{n}\right)\right) \rightarrow x_{j}$, where $c, d \in \Sigma_{\text {pub }}, j \in \tilde{n}$, and $x_{1}, \ldots, x_{n}$ are pairwise distinct variables.

- (R3): $c\left(x_{1}, \ldots, x_{n}\right) \rightarrow x_{j}$ where $c \in \Sigma_{\text {pub }}, x_{j}$ is a variable with $j \in \tilde{n}$, and $x_{i}$ is a variable or an atom for all $i \in \widetilde{n}$.

- (R4): $l \rightarrow a$ for a constant $a$.

Intuitively, the first three forms enable different types of projection of a term's arguments. Rules R1 and R2 apply a destructor $d$ to extract one of $c$ 's arguments. In rule $\mathrm{R} 1$ the destructor has two arguments. The terms $t$ and $u$ can be seen a pair of matching keys required to extract $x_{j}$. Rule R3 uses no destructor. Finally, R4 models rewriting a term to a constant. Since the rules (R1-R3) have limited depth, we call the class $\mathcal{S}$ of rewrite theories shallow subterm-convergent.

We also introduce a condition on the equations $A x$ of the rewrite theory.

Definition 1. A rewrite theory $(\Sigma, A x, R)$ is well-formed if for all $\{s, t\} \in A x$, we have (i) neither $s$ nor $t$ is a pair and (ii) top $(s)=$ top $(t)$.

We only consider equational theories that can be decomposed into a shallow subterm-convergent, well-formed rewrite theory. These are adequate to model many well-known cryptographic primitives as illustrated by the examples below.

Example 1. We model the protocols of our case studies (see Sections 1 and 4) in the rewrite theory $\mathcal{R}_{c s}=\left(\Sigma_{c s}, A x_{c s}, R_{c s}\right)$ where

$\Sigma_{c s}=\left\{\right.$ sh, pk, pri, prf, kdf, mac, $\langle\cdot, \cdot\rangle, \pi_{1}, \pi_{2},\{|\cdot|\} .,\{\cdot \mid\}^{-1},\{\cdot\} .,\{\cdot\}^{-1},[\cdot] .$, ver $\} \cup \Sigma_{c s}^{0}$

contains function symbols for: shared, public, and private long-term keys (where $\Sigma_{\text {pri }}=\{$ sh, pri $\}$ ) hash functions prf, kdf, and mac; pairs and projections; symmetric and asymmetric encryption and decryption; and signing and verification. 
The set of atoms $\Sigma_{c s}^{0}$ is specified later. The set $R_{c s}$ consists of rewrite rules for projections (type R2) and for decryption and signature verification (type R1):

$$
\begin{array}{lll}
\pi_{1}(\langle X, Y\rangle) \rightarrow X & \left\{\{|X|\}_{K} \mid\right\}_{K}^{-1} \rightarrow X & \operatorname{ver}\left([X]_{\operatorname{pri}(K)}, \operatorname{pk}(K)\right) \rightarrow X \\
\pi_{2}(\langle X, Y\rangle) \rightarrow Y & \left\{\{X\}_{\mathrm{pk}(K)}\right\}_{\operatorname{pri}(K)}^{-1} \rightarrow X &
\end{array}
$$

We have two equations in $A x_{c s}$, namely, $\exp (\exp (g, X), Y) \simeq \exp (\exp (g, Y), X)$ to model Diffie-Hellman key exchange and $\operatorname{sh}(X, Y) \simeq \operatorname{sh}(Y, X)$.

Example 2. The theory of XOR is given by the following rewrite system where the rules are of types R2, R3 and R4. The rightmost rule ensures coherence [19].

$$
\begin{array}{rlrl}
X \oplus Y & \simeq Y \oplus X & X \oplus 0 \rightarrow X & X \oplus X \oplus Y \rightarrow Y \\
(X \oplus Y) \oplus Z \simeq X \oplus(Y \oplus Z) & X \oplus X \rightarrow 0 &
\end{array}
$$

For our theoretical development, we consider an arbitrary but fixed shallow subterm-convergent and well-formed rewrite theory $(\Sigma, A x, R)$ that includes the function symbols and rewrite rules for pairing and projections.

We denote by $\operatorname{dom}(g)$ and $\operatorname{ran}(g)$ the domain and range of a function $g$. We now define well-typed substitutions, which respect subtyping.

Definition 2 (Well-typed substitutions). A substitution $\theta$ is well-typed if $\Gamma\left((X \theta) \downarrow_{R, A x}\right) \preccurlyeq \Gamma(X)$ for all $X \in \operatorname{dom}(\theta)$.

\subsection{Protocols}

For a set of terms $T$, we define the set of events $\operatorname{Evt}(T)=\{\operatorname{snd}(t), \operatorname{rcv}(t) \mid t \in T\}$ and $\operatorname{term}(e v(t))=t$ for event $e v(t)$. A role is a sequence of events from $\operatorname{Evt}(\mathcal{M})$.

Definition 3 (Protocol). A protocol is a function $P: \mathcal{V}_{\alpha} \rightarrow \operatorname{Evt}(\mathcal{M})^{*}$ mapping agent variables to roles. Let $\mathcal{M}_{P}=\operatorname{term}(\operatorname{ran}(P))$ be the set of protocol terms appearing in the roles of $P$, and let $\mathcal{V}_{P}, \mathcal{A}_{P}, \mathcal{F}_{P}$, and $\mathcal{C}_{P}$ denote the sets of variables, agents, fresh values, and constants in $\mathcal{M}_{P}$.

Example 3 ( $\left(\mathrm{KE}_{\mathrm{m}}\right.$ protocol). We formalize the $\mathrm{IKE}_{\mathrm{m}}$ protocol from Section 1 in the rewrite theory of Example 1 as follows, using upper-case (lower-case) identifiers for variables (atoms). The atoms $\Sigma_{c s}^{0}$ are composed of constants $C=$ $\{g, o, s A 1, s A 2, t S a, t S b\}$ and fresh values $F=\{n a, n b, x, y, s P I a, s P I b\}$. The variables and their types are $A, B: \alpha, G a, G b: m s g, S P I a, S P I b, N a, N b:$ nonce where nonce is a user-defined type that satisfies $\beta_{n} \preccurlyeq 0$ nonce for all $n \in F$. We show here the initiator role $A$. The responder role $B$ is dual.

$$
\begin{aligned}
\operatorname{IKE}_{\mathrm{m}}(A)= & \operatorname{snd}(s P I a, o, s A 1, \exp (g, x), n a) \cdot \operatorname{rcv}(s P I a, S P I b, s A 1, G b, N b) . \\
& \operatorname{snd}\left(s P I a, S P I b,\{|A, B, A U T H a a, s A 2, t S a, t S b|\}_{S K a}\right) . \\
& \operatorname{rcv}\left(s P I a, S P I b,\{|B, A U T H b a, s A 2, t S a, t S b|\}_{S K a}\right)
\end{aligned}
$$

where the terms $S K a=\operatorname{kdf}(n a, N b, \exp (G b, x), s P I a, S P I b)$ and

$$
\begin{aligned}
A U T H a a & =\operatorname{mac}(\operatorname{sh}(A, B), s P I a, o, s A 1, \exp (g, x), n a, N b, \operatorname{prf}(S K a, A)) \\
A U T H b a & =\operatorname{mac}(\operatorname{sh}(A, B), s P I a, S P I b, s A 1, G b, N b, n a, \operatorname{prf}(S K a, B)) .
\end{aligned}
$$

represent the initiator $A$ 's view of the session key and of the authenticators. 


$$
\frac{u \in T}{T \vdash_{E} u} \mathrm{Ax} \quad \frac{T \vdash_{E} t^{\prime} t^{\prime}={ }_{E} t}{T \vdash_{E} t} \mathrm{Eq} \quad \frac{T \vdash_{E} t_{1} \cdots \quad T \vdash_{E} t_{n}}{T \vdash_{E} f\left(t_{1}, \ldots, t_{n}\right)} \operatorname{Comp}\left(f \in \Sigma_{\text {pub }}^{\geq 1}\right)
$$

Fig. 1. Intruder deduction rules (where $\Sigma_{\text {pub }}^{\geq 1}=\Sigma^{\geq 1} \cap \Sigma_{\text {pub }}$ )

\subsection{Operational semantics}

Let $T I D$ be a countably infinite set of thread identifiers. When we instantiate a role into a thread for execution, we mark its variables and fresh values with the thread identifier $i$. We define the instantiation $t^{\# i}$ of a term $t$ for $i \in T I D$ as the term where every variable or fresh value $u$ is replaced by $u^{i}$. Constants and agents remain unchanged. Instantiation does not affect the type of a term.

We define by $T^{\sharp}=\left\{t^{\# i} \mid t \in T \wedge i \in T I D\right\}$ the set of instantiations of terms in a set $T$ and abbreviate $T^{b}=T \cup T^{\sharp}$. For example, $\mathcal{M}^{\sharp}$ is the set of instantiated message terms, which we will use to instantiate roles into threads. We define the set of network messages exchanged during protocol execution by $\mathcal{N}=\mathcal{T}\left(\mathcal{V}^{\sharp}, \mathcal{A} \cup \mathcal{F}^{\sharp} \cup \mathcal{F}^{\bullet} \cup \mathcal{C}\right)$, where $\mathcal{F}^{\bullet}=\left\{n_{k}^{\bullet} \mid n \in \mathcal{F} \wedge k \in \mathbb{N}\right\}$ is the set of attacker-generated fresh values. Note that $\mathcal{M}^{\sharp} \subseteq \mathcal{N}$. We abbreviate $\mathcal{T}=\mathcal{M} \cup \mathcal{N}$.

We use a Dolev-Yao attacker model parametrized by an equational theory $E$. Its judgements are of the form $T \vdash_{E} t$ meaning that the intruder can derive term $t$ from the set of terms $T$. The derivable judgements are defined in a standard way by the three deduction rules in Figure 1.

We define a transition system with states $(t r, t h, \sigma)$, where

- $t r$ is a trace consisting of a sequence of pairs of thread identifiers and events,

- th $: T I D \rightarrow \operatorname{dom}(P) \times \operatorname{Evt}\left(\mathcal{M}_{P}^{\sharp}\right)^{*}$ are threads executing role instances, and

$-\sigma: \mathcal{V}^{\sharp} \rightarrow \mathcal{N}$ is a well-typed ground substitution from instantiated protocol variables to network messages such that $\mathcal{V}_{P}^{\sharp} \subseteq \operatorname{dom}(\sigma)$.

The trace $t r$ as well as the executing role instance are symbolic (with terms in $\mathcal{M}^{\sharp}$ ). The separate substitution $\sigma$ instantiates these messages to (ground) network messages. The ground trace associated with such a state is $\operatorname{tr} \sigma$.

The set Init $_{P}$ of initial states of protocol $P$ contains all $(\epsilon, t h, \sigma)$ satisfying

$$
\forall i \in \operatorname{dom}(t h) . \exists R \in \operatorname{dom}(P) . t h(i)=\left(R, P(R)^{\# i}\right)
$$

where all terms in the respective protocol roles are instantiated. The substitution $\sigma$ is chosen non-deterministically in the initial state.

The rules in Figure 2 define the transitions. In both rules, the first premise states that a send or receive event heads thread $i$ 's role. This event is removed and added together with the thread identifier $i$ to the trace $t r$. The substitution $\sigma$ remains unchanged. The second premise of $R E C V$ requires that the network message $t \sigma$ matching the term $t$ in the receive event is derivable from the intruder's (ground) knowledge $I K(t r) \sigma \cup I K_{0}$. Here, $I K(t r)$ denotes the (symbolic) intruder 


$$
\begin{aligned}
& \frac{t h(i)=(R, \operatorname{snd}(t) \cdot t l)}{(t r, t h, \sigma) \rightarrow(t r \cdot(i, \operatorname{snd}(t)), t h[i \mapsto(R, t l)], \sigma)} \text { SEND } \\
& \frac{t h(i)=(R, \operatorname{rcv}(t) \cdot t l) \quad I K(t r) \sigma \cup I K_{0} \vdash_{E} t \sigma}{(t r, t h, \sigma) \rightarrow(t r \cdot(i, \operatorname{rcv}(t)), t h[i \mapsto(R, t l)], \sigma)} R E C V
\end{aligned}
$$

Fig. 2. Operational semantics

knowledge derived from a trace $t r$ as the set of terms in the send events on $t r$, i.e., $I K(t r)=\{t \mid \exists i$. $(i$, snd $(t)) \in t r\}$ and $I K_{0}$ denotes the intruder's (ground) initial knowledge. We assume $\mathcal{A} \cup \mathcal{C} \cup \mathcal{F}^{\bullet} \subseteq I K_{0}$ and $I K_{0}$ is $R, A x$-normal. Note that the $S E N D$ rule implicitly updates this intruder knowledge.

\subsection{Property language}

We use the same specification language as in [22] to express secrecy and authentication properties. Hence, we only sketch some of its elements and give examples. There are atomic formulas to express equality $(t=u)$, the secrecy of a term (secret $(t))$, the occurrence of an event $e$ by thread $i$ in the trace $(\operatorname{steps}(i, e))$, that thread $i$ executes role $R$, and the honesty of other agents in the view of a thread $i$. Quantification is allowed over thread identifier variables. To achieve attack preservation, the predicate secret $(t)$ may occur only positively.

Example 4 (Properties of $\mathrm{IKE}_{\mathrm{m}}$ ). We express the secrecy of the Diffie-Hellman key $\exp (G b, x)$ for role $A$ of the protocol $\mathrm{IKE}_{\mathrm{m}}$ of Example 3 as follows.

$$
\phi_{s}=\forall j .\left(\operatorname{role}(j, A) \wedge \operatorname{honest}(j,[A, B]) \wedge \operatorname{steps}\left(j, \operatorname{rcv}\left(t_{4}\right)\right)\right) \Rightarrow \operatorname{secret}\left(\exp \left(G b^{j}, x^{j}\right)\right) .
$$

where $t_{4}=\left\langle s P I a, S P I b,\{|B, A U T H b a, s A 2, t S a, t S b|\}_{S K a}\right\rangle$ and $\operatorname{honest}(j,[A, B])$ means that $A$ and $B$ are honest. We formalize non-injective agreement of $A$ with $B$ on the nonces $n a$ and $n b$ and the DH half-keys $\exp (g, x)$ and $\exp (g, y)$ by

$$
\begin{aligned}
\phi_{a}= & \forall j . \\
\Rightarrow & \left(\operatorname{role}(j, A) \wedge \operatorname{honest}(j,[A, B]) \wedge \operatorname{steps}\left(j, \operatorname{rcv}\left(t_{4}\right)\right)\right) \\
& \left.\left\langle A^{j}, B^{j}, n a^{j}, N b^{j}, \exp \left(g, x^{j}\right), G b^{j}\right\rangle=\left\langle A^{k}, B^{k}, N a^{k}, n b^{k}, G a^{k}, \exp \left(g, y^{k}\right)\right\rangle\right) .
\end{aligned}
$$

\section{Security protocols abstractions}

We introduce our security protocol abstractions and illustrate their usefulness on our running example. We will present two types of protocol abstractions:

Typed abstractions transform a term's structure by reordering or removing fields and by splitting or removing cryptographic operations. The same transformations are applied to all terms of a given type and its subtypes. 
Untyped abstractions complement typed ones with additional simplifications: the removal of unprotected atoms and variables and of redundant subterms.

Our main results are soundness theorems for these abstractions. They ensure that any attack on a given property of the original protocol translates to an attack on the abstracted protocol. As we will see, these results hold under certain conditions on the protocol and the property. Here, we will focus on typed abstractions, but we will also briefly introduce the untyped ones (for more details, see Appendices G.1 to G.3).

\subsection{Typed protocol abstractions}

Our typed abstractions are specified by a list of recursive equations subject to some conditions on their shape. We define their semantics in terms of a simple Haskell-style functional program. We use both pattern matching on terms and subtyping on types to select the equation to be applied to a given term. This ensures that terms of related types are transformed in a uniform manner.

Syntax Let $\mathcal{W}=\left\{\mathcal{W}_{\tau}\right\}_{\tau \in \mathcal{Y}}$ be a family of pattern variables disjoint from $\mathcal{V}$. We define the set of patterns by $\mathcal{P}=\mathcal{T}(\mathcal{W}, \emptyset)$. A pattern $p \in \mathcal{P}$ is called linear if each (pattern) variable occurs at most once in $p$. We extend the typing function $\Gamma$ to patterns by setting $\Gamma(X)=\tau$ if and only if $X \in \mathcal{W}_{\tau}$ and then lifting it homomorphically to all patterns. Our typed message abstractions are instances of the following recursive function specifications.

Definition 4. $A$ function specification $F_{f}=\left(f, E_{f}\right)$ consists of an unary function symbol $f \notin \Sigma^{1}$ and a list of equations

$$
E_{f}=\left[f\left(p_{1}\right)=u_{1}, \ldots, f\left(p_{n}\right)=u_{n}\right],
$$

where each $p_{i} \in \mathcal{P}$ is a linear pattern such that $u_{i} \in \mathcal{T}_{\Sigma \geq 1} \cup\{f\}\left(\operatorname{vars}\left(p_{i}\right)\right)$ for all $i \in \widetilde{n}$, i.e., $u_{i}$ consists of variables from $p_{i}$ and function symbols from $\Sigma^{\geq 1} \cup\{f\}$.

We use vectors (lists) of terms $\bar{t}=\left[t_{1}, \ldots, t_{n}\right]$ for $n>0$. We define $\operatorname{set}(\bar{t})=$ $\left\{t_{1}, \ldots, t_{n}\right\}$ and $\widehat{f}(\bar{t})=\left\langle f\left(t_{1}\right), \ldots, f\left(t_{n}\right)\right\rangle$, the elementwise application of a function $f$ to a vector where the result is converted to a tuple (with the convention $\langle t\rangle=t)$. We extend split to vectors by $\operatorname{split}(\bar{t})=\operatorname{split}(\operatorname{set}(\bar{t}))$. We define three sets of function symbols occurring in $R$ and $A x$ as follows.

$$
\begin{aligned}
& \mathrm{C}_{\mathrm{R}}=\left\{c \mid d\left(c\left(x_{1}, \ldots, x_{n}, t\right), u\right) \rightarrow x_{j} \in R\right\} \\
& \mathrm{C}_{\mathrm{Key}}=\bigcup\left\{c t(t) \cup \operatorname{ct}(u) \mid d\left(c\left(x_{1}, \ldots, x_{n}, t\right), u\right) \rightarrow x_{j} \in R\right\} \\
& \mathrm{C}_{\mathrm{Ax}}=\bigcup\{c t(s) \cup c t(t) \mid\{s, t\} \in A x\}
\end{aligned}
$$

The function $p p(c)$ returns the set of extractable indices of a function symbol $c$, i.e., $p p(c)=\left\{j \mid d\left(c\left(x_{1}, \ldots, x_{n}, t\right), u\right) \rightarrow x_{j} \in R\right.$ or $\left.d\left(c\left(x_{1}, \ldots, x_{n}\right)\right) \rightarrow x_{j} \in R\right\}$. 
Definition 5 (Typed abstraction). A function specification $F_{f}=\left(f, E_{f}\right)$ is $a$ typed abstraction if each equation in $E_{f}$ has the form

$$
f\left(c\left(p_{1}, \ldots, p_{n}\right)\right)=\left\langle e_{1}, \ldots, e_{d}\right\rangle
$$

where for each $i \in \widetilde{d}$ we have either

(a) $e_{i}=f(q)$ such that $q \in \operatorname{split}\left(p_{j}\right)$ for some $j \in \tilde{n}$, or

(b) $e_{i}=c\left(\widehat{f}\left(\overline{q_{1}}\right), \ldots, \widehat{f}\left(\overline{q_{n}}\right)\right)$ such that $\operatorname{set}\left(\overline{q_{j}}\right) \subseteq \operatorname{split}\left(p_{j}\right)$ for all $j \in \widetilde{n}, c \neq\langle\cdot, \cdot\rangle$, and $c \in \mathrm{C}_{\mathrm{R}}$ implies $\overline{q_{n}}=\left[p_{n}\right]$, i.e., $\widehat{f}\left(\overline{q_{n}}\right)=f\left(p_{n}\right)$.

Moreover, we require (i) for all $j \in p p(c)$ we have $\operatorname{split}\left(p_{j}\right) \subseteq Q_{j}$ where

$$
Q_{j}=\bigcup\left\{\operatorname{set}\left(\overline{q_{j}}\right) \mid \exists i \in \widetilde{d} . e_{i}=c\left(\widehat{f}\left(\overline{q_{1}}\right), \ldots, \widehat{f}\left(\overline{q_{n}}\right)\right)\right\} \cup\left\{q \mid \exists i \in \widetilde{d} . e_{i}=f(q)\right\} .
$$

and (ii) if $c \in \mathrm{C}_{\mathrm{Ax}} \cup \mathrm{C}_{\text {Key }}$ then $p_{i}=X_{i}$ : msg for all $i \in \widetilde{n}, d=1$ and $e_{1}=$ $c\left(f\left(X_{1}\right), \ldots, f\left(X_{n}\right)\right)$ is an instance of $(b)$; we say $F_{f}$ is homomorphic for $c$.

Intuitively, the abstractions can only weaken the cryptographic protection of terms, but never strengthen it. Each equation in $E_{f}$ maps a term with top-level symbol $c$ to a tuple whose components have the form (a) or (b). Form (a) allows us to pull fields out of the scope of $c$, hence removing $c$ 's protection. Using form (b) we can reorder or remove fields in each argument of $c$. Form (b) is subject to two conditions. First, we disallow this form for pairs to obtain the simple shape $f\left(\left\langle p_{1}, p_{2}\right\rangle\right)=\widehat{f}(\bar{q})$. Second, we cannot permit the reordering or removal of fields in key positions, i.e., in the last argument of $c \in \mathrm{C}_{\mathrm{R}}$. Moreover, by point (i), all fields of extractable arguments, i.e., elements of $\operatorname{split}\left(p_{j}\right)$ for $j \in p p(c)$, must be present in some $e_{i}$ and point (ii) requires that the abstraction is homomorphic for function symbols $c$ occurring in axioms and in keys $\left(c \in \mathrm{C}_{\mathrm{Ax}} \cup \mathrm{C}_{\mathrm{Key}}\right)$.

Example 5. We present a typed abstraction $F_{f}=\left(f, E_{f}\right)$ illustrating a representative selection of the possible message transformations. Suppose $X: \gamma_{c}$, $Y$ : nonce, and $Z, U, V: m s g$ and let $E_{f}$ consists of the following three equations:

$$
\begin{aligned}
f(\langle X, Y, Z\rangle) & =\langle f(Y), f(X), f(Z)\rangle \\
f(\operatorname{kdf}(X, Y, U, V)) & =\langle\operatorname{kdf}(f(X), f(Y)), \operatorname{kdf}(f(U))\rangle \\
f\left(\{X, Y, Z \mid\}_{U}\right) & \left.=\left\langle\{f(X), f(Y)\}_{f(U)}, f(Y),\{\mid f(Z)\}\right\}_{f(U)}\right\rangle
\end{aligned}
$$

The patterns' types filter the matching terms: $X$ and $Y$ only match the constant $c$ and a nonce, respectively. The first equation swaps the first two fields in $n$-tuples for $n \geq 3$. The second one splits a kdf hash into two, removing the field $V$. The last equation splits an encryption: the pair $\langle f(X), f(Y)\rangle$ and $f(Z)$ are encrypted separately with the key $f(U)$ and $f(Y)$ is pulled out of the encryption. Note that by condition (i) of Definition 5, we cannot directly remove plaintext fields from encryptions. To achieve this, we pull such fields out of encryptions to the toplevel. This may require a combination of several abstractions if there are multiple layers of cryptographic protection. At the top-level, the fields are no longer protected and can be removed using untyped abstractions. In Section 4.1, we will discuss our heuristics to determine sequences of abstractions automatically. 


$$
\begin{aligned}
& \text { fun } f(t)=\text { case } t \text { of } \\
& \quad \|_{(f(p)=u) \in E_{f}^{+}} \quad p \mid \Gamma(t) \preccurlyeq \Gamma(p) \Rightarrow u
\end{aligned}
$$

Program 1. Functional program $f$ resulting from $F_{f}=\left(f, E_{f}\right)$.

Semantics The semantics of a typed abstraction $F_{f}$ is given by the Haskellstyle functional program $f$ (Program 1). ${ }^{1}$ To ensure totality, we use the extended function specification $\left(f, E_{f}^{+}\right)=\left(f, E_{f} \cdot E_{f}^{0}\right)$, where $f\left(g\left(Z_{1}, \ldots, Z_{n}\right)\right)=$ $g\left(f\left(Z_{1}\right), \ldots, f\left(Z_{n}\right)\right) \in E_{f}^{0}$ for each $g \in \Sigma^{n}$ with $n \geq 1$ such that $Z_{i}: m s g$ for all $i \in \widetilde{n}$, and $f(Z)=Z$ with $Z: m s g$ is the last clause in $E_{f}^{0}$. We assume $E_{f}$ and $E_{f}^{0}$ do not share variables. The case statement has a clause

$$
p \mid \Gamma(t) \preccurlyeq \Gamma(p) \Rightarrow u
$$

for each equation $f(p)=u$ of $E_{f}^{+}$. Such a clause is enabled if (1) the term $t$ matches the pattern $p$, i.e., $t=p \theta$ for some substitution $\theta$, and (2) its type $\Gamma(t)$ is a subtype of $\Gamma(p)$. The first enabled clause is executed. Hence, the equations $E_{f}^{0}$ serve as fall-back clauses, which cover the terms not handled by $E_{f}$. In particular, the last clause $f(Z)=Z$ handles exactly the atoms and variables.

We extend $f$ to events, event sequences, and traces by applying $f$ to the terms they contain and to substitutions and protocols by applying $f$ to the terms in their range. Similarly, we extend $f$ to formulas $\phi$ of our property language by applying $f$ to all terms occurring in $\phi$.

Finding abstractions Finding abstractions is fully automated by our tool using a heuristic that we will describe in Section 4.1. However, the resulting abstractions can be counterintuitive. Therefore, we present here a simplified strategy that we apply to our running example below: We start by identifying the terms that appear in the $\operatorname{secret}(\cdot)$ predicates and equations of the desired properties. Then we determine the cryptographic operations that are essential to achieve these properties and try to remove all other terms and operations.

Example 6 (from $\mathrm{IKE}_{\mathrm{m}}$ to $\mathrm{IKE}_{\mathrm{m}}^{1}$ ). In order to preserve the secrecy of the $\mathrm{DH}$ key $\exp (\exp (g, x), y)$ and the agreement on $n a, n b, \exp (g, x)$, and $\exp (g, y)$, we have to keep either the mac or the symmetric encryption with $S K$ (see Examples 3 and 4 ). We want to remove as many other fields and operations as possible (e.g., prf). We choose to remove the encryption as this allows us to later remove additional fields (e.g., sA2) using untyped abstractions. We keep o in AUTHa to prevent unifiability with $A U T H b$ and hence potential false negatives. This leads

\footnotetext{
${ }^{1}$ We are overloading the symbol $f$ here, but no confusion should arise.
} 
us to the typed abstraction $F_{1}=\left(f_{1}, E_{1}\right)$ where $E_{1}$ is defined by the equations

$$
\begin{aligned}
f_{1}\left(\{|X, Y|\}_{Z}\right) & =\left\langle f_{1}(X), f_{1}(Y)\right\rangle \\
f_{1}\left(\operatorname{mac}\left(X_{1}, \ldots, X_{8}\right)\right) & =\operatorname{mac}\left(\widehat{f}_{1}\left(\left[X_{1}, X_{3}, X_{5}, X_{6}, X_{7}, X_{8}\right]\right)\right) \\
f_{1}\left(\operatorname{mac}\left(Y_{1}, \ldots, Y_{8}\right)\right) & =\operatorname{mac}\left(\widehat{f}_{1}\left(\left[Y_{1}, Y_{5}, Y_{6}, Y_{7}, Y_{8}\right]\right)\right) \\
f_{1}\left(\operatorname{kdf}\left(Z_{1}, \ldots, Z_{5}\right)\right) & =\operatorname{kdf}\left(f_{1}\left(Z_{3}\right)\right) \\
f_{1}(\operatorname{prf}(U, Z)) & =f_{1}(U)
\end{aligned}
$$

(where we omitted the homomorphic clauses for exp and $\langle\cdot, \cdot\rangle$ ) and $X: \alpha, X_{3}: \gamma_{o}$, $Y_{3}:$ nonce, $Z_{3}: \exp (m s g, m s g), U: \operatorname{kdf}(m s g)$ and all remaining pattern variables are of type $m s g$. Applying $f_{1}$ to $\mathrm{IKE}_{\mathrm{m}}$ we obtain $\mathrm{IKE}_{\mathrm{m}}^{1}$. Here is the abstracted initiator role.

$$
\begin{aligned}
S_{\mathrm{IKE}_{\mathrm{m}}^{1}}(A)= & \operatorname{snd}(s P I a, o, s A 1, \exp (g, x), n a) \cdot \operatorname{rcv}(s P I a, S P I b, s A 1, G b, N b) . \\
& \operatorname{snd}(s P I a, S P I b, A, B, A U T H a a, s A 2, t S a, t S b) \cdot \\
& \operatorname{rcv}(s P I a, S P I b, B, A U T H b a, s A 2, t S a, t S b)
\end{aligned}
$$

with $S K a=\operatorname{kdf}(\exp (G b, x)), A U T H a a=\operatorname{mac}(\operatorname{sh}(A, B), o, \exp (g, x), n a, N b, S K a)$, and $A U T H b a=\operatorname{mac}(\operatorname{sh}(A, B), G b, N b, n a, S K a)$. In a second step, we will remove most fields in the roles of $\mathrm{IKE}_{\mathrm{m}}^{1}$ using untyped abstractions.

\subsection{Soundness of typed abstractions}

To justify the soundness of our abstractions, we show that any attack on a property $\phi$ of the original protocol $P$ is reflected as an attack on the property $f(\phi)$ of the abstracted protocol $f(P)$. We decompose this into reachability preservation (RP) and an attack preservation (AP) as follows. We require that, for all reachable states $(t r, t h, \sigma)$ of $P$, there is a ground substitution $\sigma^{\prime}$ such that

(RP) $\left(f(t r), f(t h), \sigma^{\prime}\right)$ is a reachable state of $f(P)$, and

(AP) $(t r, t h, \sigma) \not \models \phi$ implies $\left(f(t r), f(t h), \sigma^{\prime}\right) \not \models f(\phi)$.

These properties will require some assumptions about the protocol $P$, the formula $\phi$, and the abstraction $f$. Before we formally state the soundness theorem, we will introduce and motivate these assumptions while sketching its proof. For the remainder of this subsection we assume arbitrary but fixed $P, \phi, F_{f}$.

We start with two basic properties of abstractions. The first one, which we call the substitution property, states that $f(t \theta)=f(t) f(\theta)$ for well-typed $R, A x$-normal substitutions $\theta$. This does not hold in general. For example, suppose $E_{f}$ contains the clauses $f\left(h\left(Y: \gamma_{c}\right)\right)=f(X)$ and $f(h(X: m s g))=h(f(X))$ in this order. Then the property is violated for $t=h(Z: m s g)$ and $\theta=[c / Z]$. Thus, we must ensure that $t$ and all its instance $t \theta$ are transformed uniformly, i.e., match the same clauses of $E_{f}$. We therefore require that (i) the patterns in $E_{f}$ must not overlap and (ii) all recursive calls of $f$ on composed terms during the transformation of $t$ are handled by the clauses of $E_{f}$, without recourse to the fall-back clauses in $E_{f}^{0}$. This is formalized in the following two definitions where we denote the set of pattern types of a list of equations $L$ by $\Pi(L)=\{\Gamma(p) \mid(f(p)=u) \in L\}$, we define $\Pi_{f}=\Pi\left(E_{f}\right)$, and let $\operatorname{Rec}\left(F_{f}, t\right)$ be the set of terms $u$ such that $f(u)$ is called in the computation of $f(t)$. 
Definition 6. A function specification $F_{f}$ is pattern-disjoint if the types in $\Pi_{f}$ are pairwise disjoint, i.e., $\Gamma\left(p_{i}\right) \downarrow \cap \Gamma\left(p_{j}\right) \downarrow=\emptyset$ for all $i, j \in \widetilde{n}$ such that $i \neq j$.

Definition 7 (Uniform domain). We define the uniform domain of $F_{f}$ by $\operatorname{udom}\left(F_{f}\right)=\left\{t \in \mathcal{T} \mid \Gamma\left(\operatorname{Rec}\left(F_{f}, t\right)\right) \subseteq \Pi_{f} \downarrow \cup \mathcal{Y}_{a t}\right\}$.

We will require that the protocol terms $t \in \mathcal{M}_{P}$ belong to this set, which ensures that their instances $t \theta$ with $R, A x$-normal substitutions $\theta$ are transformed uniformly. We henceforth assume that $F_{f}$ is pattern-disjoint. Note that the abstractions defined in Examples 5 and 6 are pattern-disjoint.

Theorem 1 (Substitution property). Let $t \in u d o m\left(F_{f}\right)$ and $\theta$ be a welltyped and $R, A x$-normal substitution. Then $f(t \theta)=f(t) f(\theta)$.

The second basic property needed in our soundness proof is that abstractions preserve equality modulo $E$. We decompose this into the preservation of $A x$ equality and of rewriting steps. Neither is preserved in general. To ensure this we need the following two definitions.

Definition 8 ( $R, A x$-closedness). $F_{f}$ is $R, A x$-closed if the following holds: $t={ }_{A x} u$ implies $\tau_{t} \preccurlyeq \tau$ if and only if $\tau_{u} \preccurlyeq \tau$, for all $R$,Ax-normal composed terms $t: \tau_{t}$ and $u: \tau_{u}$ and all $\tau \in \Pi\left(E_{f}^{+}\right)$.

We henceforth assume that $F_{f}$ is $R, A x$-closed. In Appendix F, we present a syntactic criterion for checking this. To achieve the preservation of rewriting steps under abstraction, we must ensure that, for all positions $p$ in $t$ where a rule $l \rightarrow r \in R$ is applicable, the redex $\left.t\right|_{p}$ in $t$ is transformed into a redex $f\left(\left.t\right|_{p}\right)$ in $f(t)$ that still $A x$-matches $l$. This is the purpose of the following definition.

Definition 9 ( $R, A x$-homomorphism). We say that $f$ is $R, A x$-homomorphic for a term $t$ if for all non-variable positions $p$ in $t$ and for all rules $l \rightarrow r \in R$ such that there exists a well-typed Ax-unifier of $t_{p}$ and $l$, it holds that

(i) $f$ is homomorphic for all $c \in c t(l)$,

(ii) $f$ is homomorphic for top $\left(\left.t\right|_{p^{\prime}}\right)$ and top $\left(\left.t\right|_{p^{\prime}}\right) \neq$ top $\left(l^{\prime}\right)$ for all strict prefixes $p^{\prime}$ of $p$ and rewrite rules $l^{\prime} \rightarrow r^{\prime} \in R$ such that $c t\left(l^{\prime}\right)$ is not a singleton.

We define $\operatorname{rdom}\left(F_{f}\right)$ to be the set of terms for which $f$ is $R$, Ax-homomorphic.

Many interesting protocols $P$ satisfy $\mathcal{M}_{P} \subseteq \operatorname{rdom}\left(F_{f}\right)$, including those from our case studies. Since we must also cover redexes arising by instantiating protocol terms $t \in \mathcal{M}_{P}$, this definition employs $A x$-unification rather than $A x$-matching. The definition ensures that instantiations with $R, A x$-normal substitutions and rewriting steps both preserve the membership of terms in $\operatorname{rdom}\left(F_{f}\right)$.

Theorem 2 (Equality preservation). Let $t$ and $u$ be terms such that $t, u \in$ $\operatorname{rdom}\left(F_{f}\right)$. Then $t={ }_{E} u$ implies $f(t)={ }_{E} f(u)$. 
Reachability preservation (RP) To achieve reachability preservation, we prove that every step of $P$ can be simulated by a corresponding step of $f(P)$. In particular, to simulate receive events, we show that intruder deducibility is preserved under abstractions $f$ (cf. second premise of rule $R E C V$ ), i.e.,

$$
T \theta, I K_{0} \vdash_{E} u \theta \Rightarrow f(T) f\left(\theta \downarrow_{R, A x}\right), f\left(I K_{0}\right) \vdash_{E} f(u) f\left(\theta \downarrow_{R, A x}\right) .
$$

This property is also required to show the preservation of attacks on secrecy as part of (AP). We first establish deducibility preservation for ground terms:

Theorem 3 (Deducibility preservation). Let $T \cup\{t\} \subseteq \mathcal{N}$ be a set of ground network messages such that $\mathcal{C} \subseteq T$ and $T$ is $R, A x$-normal. Then $T \vdash_{E} t$ implies $f(T) \vdash_{E} f\left(t \downarrow_{R, A x}\right)$.

We can now derive (1) by applying Theorems 3, 2 and 1 in this order, combined with applications of rule Eq and a cut property of intruder deduction. Summarizing, reachability preservation $(\mathrm{RP})$ holds for $\mathcal{M}_{P} \subseteq u \operatorname{dom}\left(F_{f}\right) \cap \operatorname{rdom}\left(F_{f}\right)$.

Attack preservation (AP) We next define and explain the conditions on formulas needed to establish attack preservation. Let

- $S e c_{\phi}$ be the set of all terms $t$ that occur in formulas secret $(t)$ in $\phi$,

- $E q_{\phi}$ be the set of pairs $(t, u)$ such that the equation $t=u$ occurs in $\phi$ and $E q$ Term $_{\phi}=\left\{t, u \mid(t, u) \in E q_{\phi}\right\}$ is the set of underlying terms, and

- Evt $t_{\phi}$ be the set of events occurring in $\phi$.

Let $E q_{\phi}^{+}$the positively occurring equations in $\phi$ and similarly for $E v t_{\phi}$.

Definition 10 (Safe formulas). $\phi$ is safe for $P$ and $f$ if

(i) $\operatorname{Sec}_{\phi} \cup E q T e r m \phi \subseteq u \operatorname{dom}\left(F_{f}\right) \cap \operatorname{rdom}\left(F_{f}\right)$,

(ii) $f(t \sigma)={ }_{E} f(u \sigma)$ implies $t \sigma=_{E} u \sigma$ for all $(t, u) \in E q_{\phi}^{+}$and for all well-typed $R, A x$-normal ground substitutions $\sigma$, and

(iii) $f(t)=f(u)$ implies $t=u$, for all $e(t) \in \operatorname{Evt}_{\phi}^{+}$and $e(u) \in \operatorname{Evt}\left(\mathcal{M}_{P}\right)$.

Condition (i) requires that $F_{f}$ is uniform and $R, A x$-homomorphic for the terms in secrecy statements and equalities. Condition (ii) expresses the injectivity of the abstraction on the terms in positively occurring equalities. This condition is required to preserve attacks on agreement properties. In other words, it prevents abstractions from fixing attacks on agreement by identifying two terms that differ in the original protocol. In Appendix F, we provide a syntactic criterion to check condition (ii) that avoids the universal quantification over substitutions. Condition (iii) is required for properties involving event orderings and steps predicates. It states that the abstraction must not identify an event occurring positively in the property with a distinct protocol event.

We now state the soundness theorem. Below, $I K_{0}$ and $I K_{0}^{\prime}$ respectively denote the intruder's initial knowledge associated with $P$ and $f(P)$. 
Theorem 4 (Soundness). Suppose $P$, $\phi$, and $F_{f}$ satisfy (i) $f\left(I K_{0}\right) \subseteq I K_{0}^{\prime}$, (ii) $F_{f}$ is pattern-disjoint and $R$, Ax-closed, (iii) $\mathcal{M}_{P} \subseteq$ udom $\left(F_{f}\right) \cap \operatorname{rdom}\left(F_{f}\right)$, and $\phi$ is safe for $P$ and $f$. Then, for all states $(t r, t h, \sigma)$ reachable in $P$, we have

1. $\left(f(t r), f(t h), f\left(\sigma \downarrow_{R, A x}\right)\right)$ is a reachable state of $f(P)$, and

2. $($ tr, th,$\sigma) \not \models \phi$ implies $\left(f(t r), f(t h), f\left(\sigma \downarrow_{R, A x}\right)\right) \not \models f(\phi)$.

\subsection{Untyped abstractions}

Typed abstractions offer a wide range of possibilities to transform cryptographic operations including subterm removal, splitting, and pulling fields outside a cryptographic operation. We complement these abstractions with two kinds of untyped abstractions that allow us to remove (1) unprotected atoms and variables of any type and (2) redundancy in the form of intruder-derivable terms. Untyped protocol abstractions are functions $g: \mathcal{T} \rightarrow \mathcal{T} \cup\{$ nil $\}$ where messages to be removed are mapped to nil. We remove events with nil arguments from the roles. Here we have to content ourselves with an example and refer the reader to Appendix G for the details and soundness results.

Atom/variable removal The removal abstraction $\operatorname{rem}_{T}: \mathcal{T} \rightarrow \mathcal{T} \cup\{$ nil $\}$ for a set $T$ of atoms or variables is defined by

$-\operatorname{rem}_{T}(u)=$ nil if $u \in T^{b}$,

$-\operatorname{rem}_{T}\left(\left\langle t_{1}, t_{2}\right\rangle\right)= \begin{cases}\operatorname{rem}_{T}\left(t_{i}\right) & \text { if } \operatorname{rem}_{T}\left(t_{3-i}\right)=\text { nil for some } i \in \widetilde{2} \\ \left\langle\operatorname{rem}_{T}\left(t_{1}\right), \operatorname{rem}_{T}\left(t_{2}\right)\right\rangle & \text { otherwise }\end{cases}$

$-\operatorname{rem}_{T}(t)=t$ for all other terms.

In order to preserve attacks, we have to restrict the removal of atoms and variables from a protocol term $t$ to fields $u \in \operatorname{split}(t)$ that appear only unprotected (clear) in $t$, i.e., such that $u \notin \operatorname{subs}(t) \backslash \operatorname{split}(t)$.

Example 7 ( $\mathrm{IKE}_{\mathrm{m}}^{1}$ to $\left.\mathrm{IKE}_{\mathrm{m}}^{2}\right)$. We use atom/variable removal to simplify the protocol IKE $\mathrm{m}_{\mathrm{m}}^{1}$. First, we recall the specification of role $A$ of $\mathrm{IKE}_{\mathrm{m}}^{1}$.

$$
\begin{aligned}
S_{\mathrm{IKE}_{\mathrm{m}}^{1}}(A)= & \operatorname{snd}(s P I a, o, s A 1, \exp (g, x), n a) \cdot \operatorname{rcv}(s P I a, S P I b, s A 1, G b, N b) . \\
& \operatorname{snd}(s P I a, S P I b, A, B, A U T H a a, s A 2, t S a, t S b) \cdot \\
& \operatorname{rcv}(s P I a, S P I b, B, A U T H b a, s A 2, t S a, t S b)
\end{aligned}
$$

We remove the role names $A$ and $B$, the constants $o, s A 1, s A 2, t S a, t S b$, the fresh value $s P I a$, and the variable $S P I b$ using an atom/variable removal abstraction. The result is the protocol $\mathrm{IKE}_{\mathrm{m}}^{2}$ whose initiator role is defined as follows.

$$
S_{\mathrm{IKE}_{\mathrm{m}}^{2}}(A)=\operatorname{snd}(\exp (g, x), n a) \cdot \operatorname{rcv}(G b, N b) \cdot \operatorname{snd}(A U T H a a) \cdot \operatorname{rcv}(A U T H b a)
$$

We also apply the typed abstraction from Example 6 and the untyped abstraction here to the properties $\phi_{s}$ and $\phi_{a}$ of Example 4. These only affect the events in the steps predicates. The relevant soundness conditions are satisfied. 
Redundancy removal A redundancy removal abstraction $r d$ enables the elimination of redundancies within each role of a protocol. Intuitively, a protocol term $t$ appearing in a role $r$ can be abstracted to $r d(t)$ if $t$ and $r d(t)$ are derivable from each other under the intruder knowledge $T$ containing the terms preceding $t$ in $r$ and the initial knowledge $I K_{0}$. For example, we can simplify $r=\operatorname{snd}(t) \cdot \operatorname{rcv}(\langle t, u\rangle)$ to $\operatorname{snd}(t) \cdot \operatorname{rcv}(u)$. In contrast to atom/variable removal, redundancy removal can also remove composed terms. It is therefore a very effective ingredient for automatic abstraction, which we describe next.

\section{Implementation and experimental results}

We have implemented our abstraction methodology for the Scyther tool and tested it on a variety of complex protocols, mainly stemming from the IKE and ISO/IEC 9798 families. Scyther is an efficient verifier for security protocols. It supports verification for both a bounded and an unbounded number of threads. Protocols are specified by a set of linear role scripts. It also supports user-defined types. These features match our setting very well.

\subsection{Abstraction heuristics}

Our tool computes a series of successively more abstract protocols. Each abstraction step consists of a typed abstraction followed by a redundancy and an atom/variable removal abstraction. A heuristic guides the automatic generation of the typed abstractions. These abstractions may be partially user-specified.

Central to our heuristic are the (sub)terms of $S e c_{\phi}$ and $E q T e r m_{\phi}$ for a given property $\phi$, which we call essential terms. The heuristic assigns security labels, c for confidentiality and a for authenticity, to cryptographic primitives as their intended security guarantees. These labels are inherited by subterms. Concretely, we label symmetric encryptions and MACs with c and a, asymmetric encryptions and hashes with c, and signatures with a. Based on this labeling, we decide which fields are pulled outside of or removed from the topmost cryptographic operations. The main criterion is that these transformations must preserve the following labeling properties of each essential term $t$ : the presence of an a label on some occurrence of $t$ and of c labels on all occurrences of $t$. The successive abstractions work from the outside to the inside of the original protocol's terms. The untyped abstractions simply remove all inessential top-level fields.

Example 8. We can simplify the term $\{|B, A U T H b a, s A 2, t S a, t S b|\}_{S K a}$ where $A U T H b a=\operatorname{mac}(\operatorname{sh}(A, B), s P I a, S P I b, s A 1, G b, N b, n a, \operatorname{prf}(S K a, B))$ of the IKE $\mathrm{I}_{\mathrm{m}}$ protocol from Example 3 in two successive abstraction steps as follows.

$$
\begin{aligned}
\{|B, A U T H b a, s A 2, t S a, t S b|\}_{S K a} & \mapsto\langle B, A U T H b a, s A 2, t S a, t S b\rangle \\
A U T H b a & \mapsto \operatorname{mac}(\operatorname{sh}(A, B), \underline{G b}, \underline{N b}, \underline{n a}, \operatorname{prf}(S K a, B))
\end{aligned}
$$

In the first step, we pull the whole plaintext out of the encryption since the security labels of essential terms (underlined) are preserved by the mac. In the 
second step, we transform $A U T H b a$ by keeping essential and removing inessential terms. Note that removing the term $u=\operatorname{prf}(S K a, B)$ or pulling it out of the mac would not preserve authenticity for the essential term $x$ inside $S K a$. In a further step, we can simplify $u$ by deleting inessential subterms and dropping prf.

Our abstractions are sound, but not complete. Therefore, we may encounter false negatives, i.e., spurious attacks. We carefully try to avoid these, for instance, by checking that abstractions do not introduce new pairs of unifiable terms. We currently do not check automatically whether an attack is spurious. Whenever an attack on a protocol $P$ is found, we proceed to analyze (only) the failed properties on the next more concrete protocol in the series of abstractions.

\subsection{Experimental results}

We have validated the effectiveness of our abstractions on 22 members of the IKE and ISO/IEC 9798 protocol families and on the PANA-AKA protocol [3]. We verify these protocols using five tools based on four different techniques: Scyther [10], CL-Atse [25], OFMC [5], SATMC [4], and ProVerif [6]. Only Scyther and ProVerif support verification of an unbounded number of threads. In Table 1 , we present here a selection of the experimental results for Scyther and refer to Appendix $\mathrm{H}$ for a complete account, including results for other tools. Our models of the IKE and ISO/IEC 9798 protocols are based on Cremers' $[8,9]$. Since Scyther uses a fixed signature with standard cryptographic primitives and no equational theories, the IKE models approximate the DH theory by oracle roles.

We mark verified properties by $\checkmark$ and falsified ones by $\times$. An entry $\checkmark / \times$ means the property holds for one role but not for the other. Each row consists of two lines, corresponding to the analysis time without (line 1) and with (line 2) abstraction for 3-8 or unboundedly many $(\infty)$ threads. The times were measured on a cluster of 12-core AMD Opteron 6174 processors with 64 GB RAM each. They include computing the abstractions (4-20 ms) and the verification itself.

Verification For 8 of the 12 original protocols that are verified, an unbounded verification attempt results in a timeout ( $\mathrm{TO}=8 \mathrm{~h} \mathrm{cpu}$ time) or memory exhaustion (ME). In 6 of these, our abstractions enabled a verification in less than 0.4 seconds and in one case in 78 seconds. However, for the first two protocols, we still get a timeout. For the large majority of the bounded verification tasks, we significantly push the bound on the number of threads and achieve massive speedups. For example, our abstractions enable the verification of the complex nested protocols IKEv2-eap and PANA-AKA. Scyther verifies an abstraction of IKEv2-eap for up to 6 threads and, more strikingly, completes the unbounded verification of the simplified PANA-AKA in under 0.1 seconds whereas it can handle only 4 threads of the original. We also achieve dramatic speedups for many other protocols, most notably for IKEv1-pk-a22, ISO/IEC 9798-2-6, and ISO/IEC 9798-3-6-2. Moreover, the verification time for many abstracted protocols increases much more slowly than for their originals. We obtain almost 


\begin{tabular}{|c|c|c|c|c|c|c|c|c|c|c|c|}
\hline protocol & $\mathrm{No}$ & $S$ & $A \mid W$ & $N$ & 3 & 4 & 5 & 6 & 7 & 8 & $\infty$ \\
\hline \multirow{2}{*}{ IKEv1-pk-a22 } & 1 & 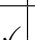 & & & 18.48 & 82.93 & 249.55 & 554.09 & 1006.04 & 1734.85 & TO \\
\hline & 1 & $\checkmark$ & & $\checkmark$ & 0.83 & 1.26 & 2.08 & 3.47 & 5.96 & 10.28 & TO \\
\hline \multirow{2}{*}{ IKEv2-eap } & & & & 8 & TO & TO & TO & TO & TO & TO & TO \\
\hline & 5 & $\checkmark$ & & $\checkmark$ & 78.35 & 798.44 & 4212.71 & 20911.20 & TO & TO & TO \\
\hline \multirow{2}{*}{ IKEv2-mac } & & & & & 1.85 & 4.91 & 6.72 & 8.07 & 8.42 & 8.49 & 8.70 \\
\hline & 5 & $\checkmark$ & & $\checkmark$ & 0.62 & 1.77 & 1.83 & 1.73 & 1.73 & 1.80 & 1.74 \\
\hline \multirow{2}{*}{ IKEv2-mactosig } & 4 & & & 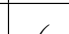 & 11.65 & 141.37 & 1075.46 & 7440.81 & TO & TO & $\mathrm{TO}$ \\
\hline & 4 & $\checkmark$ & & $\checkmark$ & 2.89 & 12.38 & 24.54 & 38.68 & 53.36 & 65.07 & 77.68 \\
\hline \multirow{2}{*}{ IKEv2-sigtomac } & 5 & 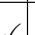 & & ( & 6.15 & 33.19 & 65.05 & 115.34 & 204.93 & 206.45 & 237.34 \\
\hline & 而 & & & $v$ & 3.59 & 12.72 & 28.44 & 44.44 & 55.11 & 66.97 & 67.15 \\
\hline \multirow{2}{*}{ IKEv1-pk-m } & 2 & & & & 48.62 & 269.92 & \begin{tabular}{|l|}
507.40 \\
\end{tabular} & 869.23 & 16254.80 & TO & TO \\
\hline & 2 & & & $x$ & 0.04 & 0.05 & 0.05 & 0.05 & 0.05 & 0.05 & TO \\
\hline \multirow{2}{*}{ IKEv1-pk-m2 } & 2 & & & & 18.26 & 274.87 & 4438.72 & TO & TO & TO & $\overline{\mathrm{TO}}$ \\
\hline & 2 & & & $x$ & 1.48 & 7.79 & 32.75 & 110.32 & 339.93 & 963.08 & TO \\
\hline \multirow{2}{*}{ IKEv1-sig-m } & 2 & & & & 0.34 & 0.45 & 0.45 & 0.45 & 0.45 & 0.46 & 0.44 \\
\hline & 2 & & & $x$ & 0.05 & 0.05 & 0.05 & 0.06 & 0.05 & 0.05 & 0.06 \\
\hline \multirow{2}{*}{ IKEv1-sig-m-perlman } & & & & & 2.86 & 13.99 & 40.78 & 67.83 & 72.08 & 72.15 & 109.03 \\
\hline & 2 & & & $x$ & 0.05 & 0.05 & 0.05 & 0.05 & 0.05 & 0.05 & 0.05 \\
\hline \multirow{2}{*}{ ISO/IEC 9798-2-5 } & 1 & 4 & & & 0.78 & 8.96 & $\mid \overline{\mid 73.87}$ & \begin{tabular}{|l|l|}
564.67 \\
\end{tabular} & $\mid 4214.22$ & TO & $\overline{\mathrm{TO}}$ \\
\hline & 1 & $\checkmark$ & & & 0.07 & 0.11 & 0.12 & 0.11 & 0.11 & 0.11 & 0.11 \\
\hline \multirow{2}{*}{ ISO/IEC 9798-2-6 } & 1 & 4 & & & 0.57 & 3.74 & 18.42 & 67.01 & 196.30 & 488.04 & 21278.58 \\
\hline & 1 & $\checkmark$ & & & 0.05 & 0.04 & 0.05 & 0.05 & 0.05 & 0.05 & 0.05 \\
\hline \multirow{2}{*}{ ISO/IEC 9798-3-6-1 } & 2 & & & . & 43.08 & 802.95 & 8903.70 & $\mathrm{ME}$ & $\mathrm{ME}$ & $\mathrm{ME}$ & $\overline{\mathrm{ME}}$ \\
\hline & 2 & & $\checkmark$ & $\checkmark$ & 0.13 & 0.18 & 0.19 & 0.19 & 0.19 & 0.19 & 0.19 \\
\hline \multirow{2}{*}{ ISO/IEC 9798-3-6-2 } & 1 & & $\checkmark$ & $\checkmark$ & 2.74 & 8.67 & 19.56 & 33.91 & 52.51 & 69.48 & 90.04 \\
\hline & & & 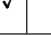 & $v$ & 0.12 & 0.15 & 0.15 & 0.15 & 0.15 & 0.15 & 0.15 \\
\hline \multirow{2}{*}{ ISO/IEC 9798-3-7-1 } & 2 & & $\checkmark$ & $\checkmark$ & 40.43 & 740.47 & 7483.36 & 16631.42 & $\mathrm{ME}$ & $\mathrm{ME}$ & $\mathrm{ME}$ \\
\hline & 2 & & $\checkmark$ & $\checkmark$ & 0.13 & 0.18 & 0.19 & 0.19 & 0.19 & 0.19 & 0.19 \\
\hline \multirow{2}{*}{ ISO/IEC 9798-3-7-2 } & 1 & & $\checkmark$ & $\checkmark$ & 2.38 & 7.71 & 16.68 & 26.99 & 35.06 & 49.49 & TO \\
\hline & & & 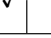 & $v$ & 0.22 & 0.32 & 0.33 & 0.33 & 0.33 & 0.33 & 0.33 \\
\hline \multirow{2}{*}{ PANA-AKA } & 5 & & & & 5769.53 & TO & TO & TO & TO & TO & TO \\
\hline & 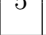 & & & $\checkmark$ & 0.10 & 0.10 & 0.10 & 0.10 & 0.10 & 0.10 & 0.10 \\
\hline
\end{tabular}

Table 1. Experimental results. The time is in seconds. No: Number of abstractions. Properties: Secrecy, Aliveness, Weak agreement, and Non-injective agreement.

constant verification times for the six ISO/IEC 9798 protocols, whereas the time significantly increases on some originals, e.g., for ISO/IEC 9798-3-6-1. For a few protocols, e.g., IKEv2-sigtomac and IKEv2-mac, the speedup is more modest.

Falsification For rows marked by $\times$, the second line corresponds to falsification time for the most abstract model, which is much faster than on the original one. For example, for 8 threads of the IKEv1-pk-m protocol, we reduce falsification time from a timeout to 0.05 seconds. In the unbounded case, the speedup factors are 7 for IKEv1-sig-m and 2180 for IKEv1-sig-m-perlman. A manual analysis of the abstract attacks shows that none of them is spurious, suggesting that our measures to prevent them are effective. We expect that fast automatic detection of spurious attacks is feasible and will affect performance only negligibly. 
Combination For the IKEv1-pk-m2 protocol, the tool verifies non-injective agreement for one role and falsifies it for the other one. Surprisingly, we obtain a remarkable speedup even though the analysis of this protocol is done three times (for two abstract and the original models). Our abstractions push the feasibility bound from 5 to 8 threads. As the property is verified very quickly for one role on the most abstract model, it needs to be analyzed only for the other role at lower abstraction levels. This explains the remarkable speedups we obtain and therefore illustrates an advantage of our abstraction mechanism in this case.

\section{$5 \quad$ Related work and conclusions}

Hui and Lowe [18] define several kinds of abstractions similar to ours with the aim of improving the performance of the CASPER/FDR verifier. They establish soundness only for ground messages and encryption with atomic keys. We work in a more general model, cover additional properties, and treat the non-trivial issue of abstracting the open terms in protocol specifications. Other works $[24,13,12]$ also propose a set of syntactic transformations, however without formally establishing their soundness. Using our results, we can, for instance, justify the soundness of the refinements in [13, Section 3.3]. Guttman [16,15] studies the preservation of security properties for a rich class of protocol transformations in the strand space model. His approach to property preservation is based on the simulation of protocol analysis steps instead of execution steps. Each such step explains the origin of a message. He does not have a syntactic soundness check.

In this work, we propose a set of syntactic protocol transformations that allows us to abstract realistic protocols and capture a large class of attacks. Unlike previous work [22,18], our theory and soundness results accommodate equational theories, untyped variables, user-defined types, and subtyping. These features allow us to accurately model protocols, capture type-flaw attacks, and adapt to different verification tools, e.g., those supporting equational theories such as ProVerif and CL-atse. We have extended Scyther with an abstraction module, which we validated it on various IKE and ISO/IEC 9798 protocols. We also tested our technique (with manually produced abstractions) on ProVerif, CL-atse, OFMC, and SATMC. Our experiments clearly show that modern protocol verifiers can substantially benefit from our abstractions, which often either enable previously unfeasible verification tasks or lead to dramatic speedups.

Our abstraction tool does not check for spurious attacks. We plan to add this functionality to complete the automatic abstraction-refinement process. We are also interested in generalizing the tool and supporting more protocol verifiers.

Acknowledgements We thank Mathieu Turuani and Michael Rusinowitch for our discussions on the topic of this paper, Cas Cremers for his help with Scyther, David Basin, Ognjen Maric, and Ralf Sasse for their careful proof-reading, and the anonymous reviewers for their useful feedback. This work is partially supported by the EU FP7-ICT-2009 Project No. 256980, NESSoS: Network of Excellence on Engineering Secure Future Internet Software Services and Systems. 


\section{References}

1. A. Armando et al.: The AVANTSSAR platform for the automated validation of trust and security of service-oriented architectures. In: Flanagan, C., König, B. (eds.) TACAS. Lecture Notes in Computer Science, vol. 7214, pp. 267-282. Springer (2012)

2. Arapinis, M., Duflot, M.: Bounding messages for free in security protocols. In: Arvind, V., Prasad, S. (eds.) FSTTCS. Lecture Notes in Computer Science, vol. 4855, pp. 376-387. Springer (2007)

3. Arkko, J., Haverinen, H.: RFC 4187: Extensible authentication protocol method for 3rd generation authentication and key agreement (EAP-AKA) (2006), http: //www.ietf.org/rfc/rfc4187

4. Armando, A., Compagna, L.: SAT-based model-checking for security protocols analysis. International Journal of Information Security 7(1), 3-32 (2008)

5. Basin, D.A., Mödersheim, S., Viganò, L.: OFMC: A symbolic model checker for security protocols. Int. J. Inf. Sec. 4(3), 181-208 (2005)

6. Blanchet, B.: An efficient cryptographic protocol verifier based on Prolog rules. In: CSFW. pp. 82-96. IEEE Computer Society (2001)

7. Cousot, P., Cousot, R.: Abstract interpretation: A unified lattice model for static analysis of programs by construction or approximation of fixpoints. In: Graham, R.M., Harrison, M.A., Sethi, R. (eds.) POPL. pp. 238-252. ACM (1977)

8. Cremers, C.: IKEv1 and IKEv2 protocol suites (2011), https://github.com/ cascremers/scyther/tree/master/gui/Protocols/IKE

9. Cremers, C.: ISO/IEC 9798 authentication protocols (2012), https://github. com/cascremers/scyther/tree/master/gui/Protocols/ISO-9798

10. Cremers, C.J.F.: The Scyther tool: Verification, falsification, and analysis of security protocols. In: Gupta, A., Malik, S. (eds.) CAV. Lecture Notes in Computer Science, vol. 5123, pp. 414-418. Springer (2008)

11. Cremers, C.J.F.: Key exchange in IPsec revisited: Formal analysis of IKEv1 and IKEv2. In: Atluri, V., Díaz, C. (eds.) ESORICS. Lecture Notes in Computer Science, vol. 6879, pp. 315-334. Springer (2011)

12. Datta, A., Derek, A., Mitchell, J.C., Pavlovic, D.: Abstraction and refinement in protocol derivation. In: Proc. 17th IEEE Computer Security Foundations Workshop (CSFW) (2004)

13. Datta, A., Derek, A., Mitchell, J.C., Pavlovic, D.: A derivation system and compositional logic for security protocols. Journal of Computer Security 13, 423-482 (2005)

14. Escobar, S., Meadows, C., Meseguer, J.: Maude-NPA: Cryptographic protocol analysis modulo equational properties. In: Aldini, A., Barthe, G., Gorrieri, R. (eds.) FOSAD. Lecture Notes in Computer Science, vol. 5705, pp. 1-50. Springer (2007)

15. Guttman, J.D.: Transformations between cryptographic protocols. In: Degano, P., Viganò, L. (eds.) ARSPA-WITS. LNCS, vol. 5511, pp. 107-123. Springer (2009)

16. Guttman, J.D.: Security goals and protocol transformations. In: Theory of Security and Applications (TOSCA). LNCS, vol. 6993. Springer (2011)

17. Harkins, D., Carrel, D.: The Internet Key Exchange (IKE). IETF RFC 2409 (Proposed Standard) (Nov 1998), http://www.ietf.org/rfc/rfc2409.txt

18. Hui, M.L., Lowe, G.: Fault-preserving simplifying transformations for security protocols. Journal of Computer Security 9(1/2), 3-46 (2001)

19. Jouannaud, J., Kirchner, H.: Completion of a set of rules modulo a set of equations. SIAM J. Comput. 15(4), 1155-1194 (1986) 
20. Kaufman, C., Hoffman, P., Nir, Y., Eronen, P.: Internet Key Exchange Protocol Version 2 (IKEv2). IETF RFC 5996 (September 2010), http://tools.ietf.org/ html/rfc5996

21. Meier, S., Schmidt, B., Cremers, C., Basin, D.: The TAMARIN prover for the symbolic analysis of security protocols. In: Sharygina, N., Veith, H. (eds.) CAV. Lecture Notes in Computer Science, vol. 8044, pp. 696-701. Springer (2013)

22. Nguyen, B.T., Sprenger, C.: Sound security protocol transformations. In: Basin, D.A., Mitchell, J.C. (eds.) POST. Lecture Notes in Computer Science, vol. 7796, pp. 83-104. Springer (2013)

23. Paulson, L.: The inductive approach to verifying cryptographic protocols. J. Computer Security 6, 85-128 (1998)

24. Pavlovic, D., Meadows, C.: Deriving secrecy in key establishment protocols. In: Proc. 11th European Symposium on Research in Computer Security (ESORICS). pp. 384-403 (2006)

25. Turuani, M.: The CL-Atse protocol analyser. In: Pfenning, F. (ed.) RTA. Lecture Notes in Computer Science, vol. 4098, pp. 277-286. Springer (2006) 


\section{A Basic lemmas about the auxiliary functions and the type system}

\section{A.1 Lemma about splitting}

Lemma 1. For all $t, u \in \mathcal{T}\left(V, \Sigma_{0}\right)$ and all substitutions $\theta$, split $(t) \subseteq \operatorname{split}(u)$ implies that split $(t \theta) \subseteq \operatorname{split}(u \theta)$

Proof. Suppose that split $(t) \subseteq \operatorname{split}(u)$ and $v \in \operatorname{split}(t \theta)$. To show that $v \in$ $\operatorname{split}(u \theta)$ we distinguish two cases:

1. There is some $t^{\prime} \in \operatorname{split}(t)$ that is not a variable and $v=t^{\prime} \theta$. Then $t^{\prime} \in$ $\operatorname{split}(u)$ and thus $t^{\prime}$ is not a pair. Since $t^{\prime}$ is neither a variable nor a pair, we have $v \in \operatorname{split}(u \theta)$.

2. There is a variable $X \in \operatorname{split}(t)$ such that $v \in \operatorname{split}(X \theta)$. Then $X \in \operatorname{split}(u)$ and thus $v \in \operatorname{split}(u \theta)$.

This completes the proof of the lemma.

\section{A.2 Lemmas about the type system}

The subtyping relation respects the types' structures.

Lemma 2. Let $\tau, \tau^{\prime} \in \mathcal{Y}$ be such that $\tau \preccurlyeq \tau^{\prime}$ and $\tau^{\prime} \neq m s g$. Then either

(i) $\tau$ and $\tau^{\prime}$ are atomic and $\tau \neq m s g$, or

(ii) $\tau$ and $\tau^{\prime}$ are composed and there is $n \geq 1$ and $g \in \Sigma^{n}$ such that $\tau=$ $g\left(\tau_{1}, \ldots, \tau_{n}\right), \tau^{\prime}=g\left(\tau_{1}^{\prime}, \ldots, \tau_{n}^{\prime}\right)$, and $\tau_{i} \preccurlyeq \tau_{i}^{\prime}$ for $i \in \widetilde{n}$.

Proof. We prove this lemma by rule induction on the derivation of $\tau \preccurlyeq \tau^{\prime}$, depending on the last rule $R$ that has been applied.

$-R=\mathrm{S}(m s g)$ : we have $\tau \in \mathcal{Y}$ and $\tau^{\prime}=m s g$, contradicting our assumption.

$-R=\mathrm{S}\left(\preccurlyeq_{0}\right)$ : we have $\tau \preccurlyeq_{0} \tau^{\prime}$. Then it is clear that both $\tau$ and $\tau^{\prime}$ are atomic and $\tau \neq m s g$ by the definition of $\preccurlyeq_{0}$.

$-R=\mathrm{S}($ refl $)$ : we have $\tau=\tau^{\prime}$ and thus the conclusion holds trivially.

$-R=\mathrm{S}($ trans $)$ : here, there is a $\tau^{\prime \prime}$ such that $\tau \preccurlyeq \tau^{\prime \prime}$ and $\tau^{\prime \prime} \preccurlyeq \tau^{\prime}$. Since $\tau^{\prime} \neq m s g$, we derive (i) or (ii) from the induction hypothesis for $\tau^{\prime \prime} \preccurlyeq \tau^{\prime}$ to for $\tau^{\prime \prime}$ and $\tau$. In both cases, we have $\tau^{\prime \prime} \neq m s g$. Therefore, we can also apply the induction hypothesis to $\tau \preccurlyeq \tau^{\prime \prime}$. Hence, we either have that $\tau, \tau^{\prime \prime}$, and $\tau^{\prime}$ are all atomic and $\tau \neq m s g$ or they all have the same top-level constructor $g$ and the arguments of $\tau$ and $\tau^{\prime \prime}$ and of $\tau^{\prime \prime}$ and $\tau^{\prime}$ are in the subtyping relation and we conclude by applying $\mathrm{S}$ (trans) on the argument types.

$-R=\mathrm{S}\left(\Sigma^{n}\right)$ : In this case, the conclusion (ii) follows directly from the rules' premises and conclusions.

The following lemma states that well-typed substitutions respect types. 
Lemma 3. Let $\theta$ be an $R, A x$-normal substitution that is well-typed. Then for all terms $t \in \mathcal{T}$, we have $\Gamma(t \theta) \preccurlyeq \Gamma(t)$.

Proof. The proof is proceeded by induction on $t$.

- If $t$ is an atom then $t \theta=t$ and thus the lemma holds trivially.

- If $t$ is a variable $X$ then we distinguish two cases. If $X \notin \operatorname{dom}(\theta)$ then we have $X \theta=X$ and this case holds trivially. Otherwise, we have $\Gamma(X \theta) \preccurlyeq \Gamma(X)$, since $\theta$ is well-typed and $R, A x$-normal.

- If $t=c\left(t_{1}, \ldots, t_{n}\right)$ for some $c \in \Sigma^{n}$ and $n \geq 1$ then we have $t \theta=c\left(t_{1} \theta, \ldots, t_{n} \theta\right)$. Moreover, by induction hypothesis, we have $\Gamma\left(t_{i} \theta\right) \preccurlyeq \Gamma\left(t_{i}\right)$ for all $i \in \widetilde{n}$. This yields $\Gamma(t \theta) \preccurlyeq \Gamma(t)$ as required. 


\section{B Basic properties of typed abstractions}

In this section, we prove several properties of typed abstractions. First, we show that two terms whose types are in a subtyping relation must be transformed by the same clause. Second, we describe the shapes of transformed terms in different cases. At the end, we prove that type inference is preserved under abstractions.

\section{B.1 Uniform matching}

The following lemma states that a term $t$ matches a linear pattern $p$ whenever $t$ 's type is a subtype of $p$ 's type.

Lemma 4. Let $p \in \mathcal{P}$ be a linear pattern. Then, for all $t \in \mathcal{T}$ such that $\Gamma(t) \preccurlyeq$ $\Gamma(p)$ there exists a substitution $\sigma: \operatorname{vars}(p) \rightarrow \mathcal{T}$ such that $p \sigma=t$.

Proof. We prove the lemma by induction on the structure of $p$. Below we use the abbreviations $\tau=\Gamma(t)$ and $\pi=\Gamma(p)$.

- If $p$ is a pattern variable, then we define $\sigma=\{t / p\}$, hence $p \sigma=t$.

- If $p=g\left(p_{1}, \ldots, p_{n}\right)$ for $g \in \Sigma^{n}, n \geq 1$ then since $\Gamma(p)=\pi$, there exists $\pi_{1}, \ldots, \pi_{n}$ such that

$$
\pi=g\left(\pi_{1}, \ldots, \pi_{n}\right) \text { and } \Gamma\left(p_{i}\right)=\pi_{i} \text { for } i \in \widetilde{n}
$$

Since $\tau \preccurlyeq \pi$, by Lemma 2 , we have

$$
\tau=g\left(\tau_{1}, \ldots, \tau_{n}\right) \text { and } \tau_{i} \preccurlyeq \pi_{i} \text { for } i \in \widetilde{n} \text {. }
$$

Since $\Gamma(t)=\tau$ and $\tau$ is composed, $t$ is not a variable. Therefore, we have

$$
t=g\left(t_{1}, \ldots, t_{n}\right) \text { and } \Gamma\left(t_{i}\right)=\tau_{i} \text { for } i \in \widetilde{n} .
$$

Hence, by induction hypothesis, there are $\sigma_{i}: \operatorname{vars}\left(p_{i}\right) \rightarrow \mathcal{T}$ such that $t_{i}=$ $p_{i} \sigma_{i}$ for $i \in \tilde{n}$. Since $p$ is linear, we can thus define $\sigma: \operatorname{vars}(p) \rightarrow \mathcal{T}$ by $\sigma=\bigcup_{i=1}^{n} \sigma_{i}$. Hence, we obtain $p \sigma=t$.

This completes the proof of the lemma.

Lemma 5 (Uniform matching). Let $E_{f}=\left[f\left(p_{1}\right)=u_{1}, \ldots, f\left(p_{n}\right)=u_{n}\right]$ and

$$
\text { matches }(t)=\left\{i \in \widetilde{n} \mid \exists \theta . t=p_{i} \theta \wedge \Gamma(t) \preccurlyeq \Gamma\left(p_{i}\right)\right\} \text {. }
$$

Then, for all $t, t^{\prime} \in \mathcal{T}$ with $\Gamma\left(t^{\prime}\right) \preccurlyeq \Gamma(t)$, we have

(i) matches $(t) \subseteq$ matches $\left(t^{\prime}\right)$,

(ii) matches $(t)=$ matches $\left(t^{\prime}\right)=\{i\}$ for some $i \in \widetilde{n}$ if $\Gamma(t) \in \Pi_{f} \downarrow$.

In particular, matches $(t)=$ matches $(\Gamma(t))$ for all terms $t \in \mathcal{T}$. 
Proof. Let $t, t^{\prime} \in \mathcal{T}, t: \tau, t^{\prime}: \tau^{\prime}$, and $\tau^{\prime} \preccurlyeq \tau$. To see (i), suppose $i \in$ matches $(t)$, i.e., $t=p_{i} \theta$ and $\Gamma(t) \preccurlyeq \Gamma\left(p_{i}\right)$ for some substitution $\theta$. Since $\Gamma\left(t^{\prime}\right) \preccurlyeq \Gamma(t)$, we also have $\Gamma\left(t^{\prime}\right) \preccurlyeq \Gamma\left(p_{i}\right)$ and hence $i \in$ matches $\left(t^{\prime}\right)$. This shows (i).

To see (ii), we first derive $\tau^{\prime} \in \Pi_{f} \downarrow$ from the assumptions $\tau \in \Pi_{f} \downarrow$ and $\tau^{\prime} \preccurlyeq \tau$. Therefore, there are $i, j \in \widetilde{n}$ and $\left\{\pi_{i}, \pi_{j}\right\} \subseteq \Pi_{f}$ such that $\tau \preccurlyeq \pi_{i}$ and $\tau^{\prime} \preccurlyeq \pi_{j}$. Moreover, $i$ and $j$ are unique since $F_{f}$ is pattern-disjoint. By Lemma 4 there are substitutions $\theta$ and $\theta^{\prime}$ (with domains $\operatorname{vars}\left(p_{i}\right)$ and $\operatorname{vars}\left(p_{j}\right)$ ) such that $t=p_{i} \theta$ and $t^{\prime}=p_{j} \theta^{\prime}$. Hence, matches $(t)=\{i\}$ and matches $\left(t^{\prime}\right)=\{j\}$. Using the result in (i) derive $i=j$ as required.

\section{B.2 Shape lemma and termination}

Lemma 6 (Shape lemma). If $t \in \mathcal{T}$ then the following holds

(i) If $t$ is a variable or an atom, then $f(t)=t$.

(ii) If $t=c\left(t_{1}, \ldots, t_{n}\right), c \in \Sigma^{n} \backslash\left(\mathrm{C}_{\mathrm{Ax}} \cup \mathrm{C}_{\text {Key }}\right)$ then we have

$$
f\left(c\left(t_{1}, \ldots, t_{n}\right)\right)=\left\langle u_{1}, \ldots, u_{d}\right\rangle
$$

for some $d>0$ and for all $i \in \widetilde{d}, u_{i}$ is one of the following forms:

(a) $u_{i}=c\left(\widehat{f}\left(\overline{v_{1}}\right), \ldots, \widehat{f}\left(\overline{v_{n}}\right)\right)$ such that

$$
\begin{aligned}
& \operatorname{split}\left(\overline{v_{j}}\right) \subseteq \operatorname{split}\left(t_{j}\right) \text { for all } j \in \tilde{n}, c \neq\langle\cdot, \cdot\rangle \text {, and } \\
& c \in \mathrm{C}_{\mathrm{R}} \Rightarrow \overline{v_{n}}=\left[t_{n}\right]
\end{aligned}
$$

(b) $u_{i}=f(v)$ such that $\operatorname{split}(v) \subseteq \operatorname{split}\left(t_{j}\right)$ for some $j \in \widetilde{n}$.

and it holds that

$$
\begin{aligned}
& \forall j \in \operatorname{pp}(c) . \operatorname{split}\left(t_{j}\right) \subseteq \operatorname{split}(P(j)) \\
& \forall j \in \widetilde{n} . P(j) \subseteq \operatorname{subs}(t) \backslash\{t\}
\end{aligned}
$$

where

$$
\begin{gathered}
P(j)=\bigcup\left\{\operatorname{set}\left(\overline{v_{j}}\right) \mid c\left(\widehat{f}\left(\overline{v_{1}}\right), \ldots, \widehat{f}\left(\overline{v_{n}}\right)\right) \in\left\{u_{1}, \ldots, u_{d}\right\}\right\} \\
\bigcup\left\{v \mid f(v) \in\left\{u_{1}, \ldots, u_{d}\right\}\right\}
\end{gathered}
$$

(iii) If $t=c\left(t_{1}, \ldots, t_{n}\right)$ for $c \in \Sigma^{n} \cap\left(\mathrm{C}_{\mathrm{Ax}} \cup \mathrm{C}_{\mathrm{Key}}\right)$ and $n \geq 1$ then

$$
f(t)=c\left(f\left(t_{1}\right), \ldots, f\left(t_{n}\right)\right)
$$

Proof. We prove this lemma by case distinction on the shape of the term $t \in \mathcal{T}$. We know that there exists the first pattern $f(p)=u$ in the list $E_{f}^{1}$ such that $\Gamma(t) \preccurlyeq \Gamma(p)$. By Lemma 4, there is a substitution $\theta$ such that $p \theta=t$. Hence, by Program 1, we have

$$
f(t)=u \theta
$$

Case (i) where $t$ is a variable or an atom follows immediately from Program 1 and the definition of $E_{f}^{0}$. Suppose $t$ is composed. We distinguish the following cases. 
- $t=c\left(t_{1}, \ldots, t_{n}\right)$ and $c \in \Sigma^{n} \backslash\left(\mathrm{C}_{\mathrm{Ax}} \cup \mathrm{C}_{\text {Key }}\right)$ : since $p$ is not a pattern variable and $t=p \theta$, we must have $p=c\left(p_{1}, \ldots, p_{n}\right)$. By Definition 5 , we have $f(p)=$ $\left\langle e_{1}, \ldots, e_{d}\right\rangle$ for some $d>0$ and for all $i \in \widetilde{d}, e_{i}$ is one of the following forms:

1. $e_{i}=c\left(\widehat{f}\left(\overline{q_{1}}\right), \ldots, \widehat{f}\left(\overline{q_{n}}\right)\right)$ such that

$$
\begin{aligned}
& \operatorname{set}\left(\overline{q_{j}}\right) \subseteq \operatorname{split}\left(p_{j}\right) \text { for all } j \in \widetilde{n}, c \neq\langle\cdot, \cdot\rangle, \text { and } \\
& c \in \mathrm{C}_{\mathrm{R}} \Rightarrow \overline{q_{n}}=\left[p_{n}\right]
\end{aligned}
$$

2. $e_{i}=f(q)$ such that $q \in \operatorname{split}\left(p_{j}\right)$ for some $j \in \widetilde{n}$.

and it holds that $\forall j \in p p(c)$. split $\left(p_{j}\right) \subseteq Q(j)$ where

$$
\begin{gathered}
Q(j)=\bigcup\left\{\operatorname{set}\left(\overline{q_{j}}\right) \mid c\left(\widehat{f}\left(\overline{q_{1}}\right), \ldots, \widehat{f}\left(\overline{q_{n}}\right)\right) \in\left\{e_{1}, \ldots, e_{d}\right\}\right\} \\
\bigcup\left\{q \mid f(q) \in\left\{e_{1}, \ldots, e_{d}\right\}\right\}
\end{gathered}
$$

Let $u_{i}=e_{i} \theta$ for all $i \in \widetilde{d}$. For each $i \in \widetilde{d}$, we distinguish two cases depending on the shape of $e_{i}$.

- If $e_{i}=c\left(\widehat{f}\left(\overline{q_{1}}\right), \ldots, \widehat{f}\left(\overline{q_{n}}\right)\right)$ for some vectors $\overline{q_{1}}, \ldots, \overline{q_{n}}$, then let $\overline{v_{i}}=\overline{q_{i}} \theta$. We obtain that $u_{i}=c\left(\widehat{f}\left(\overline{v_{1}}\right), \ldots, \widehat{f}\left(\overline{v_{n}}\right)\right)$. From the fact that

$$
\begin{aligned}
& \operatorname{set}\left(\overline{q_{j}}\right) \subseteq \operatorname{split}\left(p_{j}\right) \text { for all } j \in \widetilde{n}, \\
& c \in \mathrm{C}_{\mathrm{R}} \Rightarrow \overline{q_{n}}=\left[p_{n}\right], \text { and } p_{j} \theta=t_{j} \text { for all } j \in \widetilde{n} .
\end{aligned}
$$

we derive that $c \in \mathrm{C}_{\mathrm{R}} \Rightarrow \overline{v_{n}}=\left[t_{n}\right]$. Moreover, we also have

$$
\operatorname{split}\left(\overline{v_{j}}\right) \subseteq \operatorname{split}\left(t_{j}\right) \text { for all } j \in \tilde{n} .
$$

- If $e_{i}=f(q)$ and $q \in \operatorname{split}\left(p_{j}\right)$ for some $j \in \widetilde{n}$, then let $v=q \theta$. We derive that $\operatorname{split}(v) \subseteq \operatorname{split}\left(p_{j} \theta\right)$. Since $p_{j} \theta=t_{j}$, we obtain that

$$
\operatorname{split}(v) \subseteq \operatorname{split}\left(t_{j}\right)
$$

It remains to show that

$$
\begin{aligned}
& \forall j \in p p(c) . \operatorname{split}\left(t_{j}\right) \subseteq \operatorname{split}(P(j)) \\
& \forall j \in \widetilde{n} . P(j) \subseteq \operatorname{subs}(t) \backslash\{t\}
\end{aligned}
$$

To see the first point, let $j \in p p(c)$. By Definition 5 , we have $\operatorname{split}\left(p_{j}\right) \subseteq Q(j)$. This implies $\operatorname{split}\left(p_{j} \theta\right) \subseteq \operatorname{split}(Q(j) \theta)$. Since $p_{j} \theta=t_{j}$ and $P_{j}=Q_{j} \theta$, we obtain $\operatorname{split}\left(t_{j}\right) \subseteq P(j)$ as required. The second point follows immediately from (3) and (4).

$-t=c\left(t_{1}, \ldots, t_{n}\right)$ for $c \in \Sigma^{n} \cap\left(\mathrm{C}_{\mathrm{Ax}} \cup \mathrm{C}_{\mathrm{Key}}\right)$ and $n \geq 1$ : Since $p \theta=t$ and $p$ is not a pattern-variable, we must have $p=c\left(q_{1}, \ldots, q_{n}\right)$ and $q_{i} \theta=t_{i}$ for all $i \in \widetilde{n}$. By Definition 5, we have

$$
f\left(p_{i}\right)=c\left(f\left(q_{1}\right), \ldots, f\left(q_{n}\right)\right)
$$

Hence we derive $f(t)=u_{i} \theta=c\left(f\left(t_{1}\right), \ldots, f\left(t_{n}\right)\right)$ as required.

This completes the proof of the lemma. 
Proposition 1 (Termination). The function $f$ defined by Program 1 terminates on all terms $t \in \mathcal{T}$.

Proof. We prove this by induction on the size of $t$. If $\Gamma(t)$ is an atom then the termination of $f(t)$ is immediate. If $\Gamma(t)$ is composed then from Lemma 6 we know that that $f$ is called recursively on subterms of $t$. Hence, these calls terminate by the induction hypothesis. Therefore, $f(t)$ also terminates. This completes the proof of the proposition.

Next, we prove that all abstracted protocols are protocols. This result enables chaining different abstractions to obtain more complex one.

Proposition 2. $f(P)$ is also a protocol.

Proof. Note that $f$ maps roles to roles and is an identity on variables. Hence by Definition 3, it is clear that $f(t)$ is a protocol.

\section{B.3 Lemma about abstracted types}

Lemma 7. Let $\sigma$ be an $R, A x$-normal ground substitution that is well-typed. Then $f(\sigma)$ is well-typed.

Proof. Let $X \in \operatorname{dom}(f(\sigma))$. Then we have $X \in \operatorname{dom}(\sigma)$ and $f(X)=X$. Since $\sigma$ is $R, A x$-normal, so is $X \sigma$. Let $t$ be a term such that $t=_{A x}(X f(\theta)) \downarrow_{R, A x}$. We need to show that $\Gamma(t) \preccurlyeq \Gamma(X)$. We consider two cases.

- If $\Gamma(X)=m s g$ then it is trivial that $\Gamma(t) \preccurlyeq \Gamma(X)$.

- If $\Gamma(X)=\tau$ for an atomic type $\tau$, then since $\sigma$ is well-typed and $X \sigma$ is $R, A x$-normal, it follows that $X \sigma$ is an atom. Thus, we have $f(X \sigma)=$ $X f(\sigma)=X \sigma$. Hence $t=X \sigma$. This implies $\Gamma(t) \preccurlyeq \Gamma(X)$ as required.

This completes the proof of the lemma.

\section{B.4 Lemma about splitting and intruder deducibility}

Lemma 8. Let $t, u \in \mathcal{T}$ such that $\operatorname{split}(u) \subseteq \operatorname{split}(t)$. Then we have

$$
\operatorname{split}(f(u)) \subseteq \operatorname{split}(f(t)) .
$$

Proof. We proceed by induction on $|u|+|t|$.

- If $|\operatorname{split}(u)|+|\operatorname{split}(t)|=2$ then $\operatorname{split}(u) \subseteq \operatorname{split}(t)$ implies that $u=t$. Thus the lemma holds for this case.

- Now we assume that $|\operatorname{split}(u)|+|\operatorname{split}(t)|>2$. There are two cases.

- If $u$ is not a pair then $\operatorname{split}(u)=\{u\}$. Hence we have

$$
u \in \operatorname{split}(t)
$$

Since $|\operatorname{split}(u)|+|\operatorname{split}(t)|>2$, we have $t=\left\langle u_{1}, u_{2}\right\rangle$. Hence by Lemma 6 , we have $f(t)=\widehat{f}(\bar{v})$ for some vector $\bar{v}$ such that $\operatorname{split}(t)=\operatorname{split}(\bar{v})$. 
By (5), there is $t^{\prime} \in \operatorname{set}(\bar{v})$ such that $u \in \operatorname{split}\left(t^{\prime}\right)$. Moreover, we have $\left|t^{\prime}\right|<|t|$. Thus by induction hypothesis, we have

$$
\operatorname{split}(f(u)) \subseteq \operatorname{split}\left(f\left(t^{\prime}\right)\right) .
$$

Since $\operatorname{split}\left(f\left(t^{\prime}\right)\right) \subseteq \operatorname{split}(f(t))$, this implies that

$$
\operatorname{split}(f(u)) \subseteq \operatorname{split}(f(t)) .
$$

- If $u=\left\langle u_{1}, u_{2}\right\rangle$ then by Lemma 6 , we have that $f(u)=\widehat{f}(\bar{r})$ for some vector $\bar{r}$ of length $m$ such that $\operatorname{split}(u)=\operatorname{split}(\bar{r})$. Since split $(u) \subseteq \operatorname{split}(t)$, we have $\operatorname{split}\left(r_{i}\right) \subseteq \operatorname{split}(t)$ for all $i \in \widetilde{m}$. Moreover, we also have that $\left|r_{i}\right|<|u|$. Hence by induction hypothesis, we have $\operatorname{split}\left(f\left(r_{i}\right)\right) \subseteq$ $\operatorname{split}(f(t))$. Therefore, we obtain split $(f(u)) \subseteq \operatorname{split}(f(t))$ as required.

This completes the proof of the lemma.

The following lemma is an immediate corollary of Lemma 8.

Corollary 1. Let $t \in \mathcal{T}$ and $u \in \operatorname{split}(t)$. Then we have

$$
f(t) \vdash_{E} f(u)
$$

The following lemma shows that if the intruder learns all the transformed components of a term, he can also learn the transformed term.

Lemma 9. Let $T \cup\{u\} \subseteq \mathcal{T}$. Suppose $T \vdash_{E} f(t)$ for all $t \in \operatorname{split}(u)$. Then $T \vdash_{E} f(u)$.

Proof. We prove the lemma by induction on the size of $u$. If $u$ is not a pair then $\operatorname{split}(u)=\{u\}$ and $T \vdash_{E} f(u)$ follows immediately from the assumption. Otherwise, $u=\left\langle u_{1}, u_{2}\right\rangle$. Then, by Lemma 6 , we derive that

$$
f(u)=\widehat{f}(\bar{r})
$$

for some vector $\bar{r}=\left[r_{1}, \ldots, r_{m}\right]$ such that $\operatorname{split}(\bar{r})=\operatorname{split}(u)$ and $\operatorname{set}(\bar{r}) \subseteq$ $\operatorname{subs}(u) \backslash\{u\}$. Since $u$ is $R, A x$-normal, so are the $r_{i}$. Let $i \in \widetilde{m}$. By assumption and since $\operatorname{split}\left(r_{i}\right) \subseteq \operatorname{split}(u)$, we have $T \vdash_{E} f(t)$ for all $t \in \operatorname{split}\left(r_{i}\right)$. Since $r_{i} \in \operatorname{subs}(u) \backslash\{u\}$, we obtain $T \vdash_{E} f\left(r_{i}\right)$ from the induction hypothesis. Hence, the desired $T \vdash_{E} f(u)$ follows from $T \vdash_{E} f\left(r_{i}\right)$ for all $i \in \widetilde{m}$.

The following lemma is a consequence of the two previous lemmas.

Lemma 10. For all terms $t, u \in \mathcal{T}$, we have split $(t) \subseteq \operatorname{split}(u)$ implies that $f(u) \vdash_{E} f(t)$.

Proof. By Corollary 1, we have $f(u) \vdash_{E} f(p)$ for all $p \in \operatorname{split}(u)$. Moreover, since $\operatorname{split}(t) \subseteq \operatorname{split}(u)$, we have $f(u) \vdash_{E} f(q)$ for all $q \in \operatorname{split}(t)$. Hence, by Lemma 9, we have $f(u) \vdash_{E} f(t)$. 


\section{Substitution property}

Theorem (Substitution property; Justification of Theorem 1). Let $t \in$ $u \operatorname{dom}\left(F_{f}\right)$ and $\theta$ be a well-typed and $R, A x$-normal substitution. Then $f(t \theta)=$ $f(t) f(\theta)$.

Proof. We prove the theorem by induction on the size of $t$. Suppose $E_{f}=$ $\left[f\left(p_{1}\right)=u_{1}, \ldots, f\left(p_{n}\right)=u_{n}\right]$ and let $t$ be a term such that $t \in u \operatorname{dom}\left(F_{f}\right)$. We distinguish two cases. If $\Gamma(t)=m s g$ then $t$ is a variable and thus $f(t)=t$ using the final identity fall-back clause of $E_{f}^{0}$. It follows that $f(t) f(\theta)=t f(\theta)=f(t \theta)$ as required. Otherwise, we have $\Gamma(t) \neq m s g$. Let $t: \tau$ and $t \theta: \tau^{\prime}$. Then, we have $\tau^{\prime} \preccurlyeq \tau$ by Lemma 3. Since $t \in u \operatorname{dom}\left(F_{f}\right)$, we have $\tau \in \Pi_{f} \downarrow$. Hence, by Lemma 5 , $t$ and $t \theta$ are abstracted in the same way.

Since $t \in u \operatorname{dom}\left(F_{f}\right)$, we derive that there exists a unique pattern $(f(p)=$ $u) \in E_{f}$ and substitutions $\theta^{\prime}$ and $\theta^{\prime \prime}$ such that $p \theta^{\prime}=t$ and $p \theta^{\prime \prime}=t \theta$. Thus, we also have $t \theta=p \theta^{\prime \prime}=p \theta^{\prime} \theta$. By Program 1 (modulo renamings), we have

$$
f(t)=u\left[f / f_{0}\right] \theta^{\prime} \text { and } \quad f(t \theta)=u\left[f / f_{0}\right] \theta^{\prime} \theta .
$$

We distinguish two base cases.

$-u=p$ and $t=a$ is an atom. Then we obtain that $f(a \theta)=f(a)=a=$ $a f(\theta)=f(a) f(\theta)$ as required.

$-u=p$ and $t=X$ is a variable. Then we have $f(X)=X$. Let us consider two cases:

- If $X \in \operatorname{dom}(\theta)$, then we have

$$
f(X \theta)=X f(\theta)=f(X) f(\theta) .
$$

- If $X \notin \operatorname{dom}(\theta)$, then since $\operatorname{dom}(f(\theta))=\operatorname{dom}(\theta)$, we have $X \notin \operatorname{dom}(f(\theta))$. Hence, we have

$$
f(X \theta)=f(X)=X=X f(\theta)=f(X) f(\theta) .
$$

For the inductive cases, note that recursive calls of $f$ have subterms of $t$ as arguments by Lemma 6 . Moreover, since $t \in u d o m\left(F_{f}\right)$, we also have $t^{\prime} \in$ $u \operatorname{dom}\left(F_{f}\right)$ for each term $t^{\prime}$ occurring as the argument of a recursive call of $f$ in the computation of $f(t)$. This enables the application of the induction hypotheses (IH) below. We distinguish the following cases.

$-p=c\left(p_{1}, \ldots, p_{n}\right)$ for $c \in \Sigma^{n} \backslash\left(\mathrm{C}_{\mathrm{Ax}} \cup \mathrm{C}_{\mathrm{Key}}\right), n \geq 1$. In this case, we have

$$
f\left(c\left(p_{1}, \ldots, p_{n}\right)\right)=\left\langle e_{1}, \ldots, e_{d}\right\rangle
$$

for some $d>0$ and for all $i \in \widetilde{d}, e_{i}$ is one of the following forms:

1. $e_{i}=c\left(\widehat{f}\left(\overline{q_{1}}\right), \ldots, \widehat{f}\left(\overline{q_{n}}\right)\right)$ such that

$$
\begin{aligned}
& \operatorname{set}\left(\overline{q_{j}}\right) \subseteq \operatorname{split}\left(p_{j}\right) \text { for all } j \in \tilde{n}, c \neq\langle\cdot, \cdot\rangle, \text { and } \\
& c \in \mathrm{C}_{\mathrm{R}} \Rightarrow \overline{q_{n}}=\left[p_{n}\right]
\end{aligned}
$$


2. $e_{i}=f(q)$ such that $q \in \operatorname{split}\left(p_{i}\right)$ for some $j \in \widetilde{n}$.

and it holds that $\forall j \in p p(c)$. split $\left(p_{j}\right) \subseteq Q(j)$ where

$$
\begin{gathered}
Q(j)=\bigcup\left\{\operatorname{set}\left(\overline{q_{j}}\right) \mid c\left(\widehat{f}\left(\overline{q_{1}}\right), \ldots, \widehat{f}\left(\overline{q_{n}}\right)\right) \in\left\{e_{1}, \ldots, e_{d}\right\}\right\} \\
\bigcup\left\{q \mid f(q) \in\left\{e_{1}, \ldots, e_{d}\right\}\right\}
\end{gathered}
$$

Therefore, we have

$$
\begin{aligned}
f(t) & =\left\langle e_{1} \theta^{\prime}, \ldots, e_{d} \theta^{\prime}\right\rangle \\
f(t \theta) & \left.=\left\langle e_{1} \theta^{\prime} \theta, \ldots, e_{d} \theta^{\prime} \theta\right)\right\rangle
\end{aligned}
$$

This implies $f(t) f(\theta)=\left\langle e_{1} \theta^{\prime} f(\theta), \ldots, e_{d} \theta^{\prime} f(\theta)\right\rangle$. To see that $f(t \theta)=f(t) f(\theta)$, it is sufficient to show that $e_{i} \theta^{\prime} \theta=e_{i} \theta^{\prime} f(\theta)$ for all $i \in \widetilde{d}$. Let $i \in \widetilde{d}$, we distinguish two cases.

- If $e_{i}=c\left(\widehat{f}\left(\overline{q_{1}}\right), \ldots, \widehat{f}\left(\overline{q_{n}}\right)\right)$ then we have

$$
e_{i} \theta^{\prime} \theta=c\left(\widehat{f}\left(\overline{q_{1}} \theta^{\prime} \theta\right), \ldots, \widehat{f}\left(\overline{q_{n}} \theta^{\prime} \theta\right)\right)
$$

Since $\operatorname{set}\left(\overline{q_{j}}\right) \subseteq \operatorname{split}\left(p_{j}\right)$ for all $j \in \widetilde{n}$, we derive that $\operatorname{set}\left(\overline{q_{j}} \theta^{\prime}\right) \subseteq$ $\operatorname{subs}\left(p_{j} \theta^{\prime}\right)$ for all $j \in \widetilde{n}$. Moreover, we have $p_{j} \theta^{\prime} \in \operatorname{subs}(t) \backslash\{t\}$. Therefore, by induction hypothesis, we derive that $\widehat{f}\left(\overline{q_{j}} \theta^{\prime} \theta\right)=\widehat{f}\left(\overline{q_{j}} \theta^{\prime}\right) f(\theta)$ for all $j \in \widetilde{n}$. Note that for all $j \in \widetilde{n}$, we have $\widehat{f}\left(\overline{q_{j}} \theta^{\prime}\right)=\widehat{f}\left(\overline{q_{j}}\right) \theta^{\prime}$. Hence, we conclude that $e_{i} \theta^{\prime} \theta=e_{i} \theta^{\prime} f(\theta)$ as desired.

$-p=c\left(p_{1}, \ldots, p_{n}\right)$ for $c \in \Sigma^{n} \cap\left(\mathrm{C}_{\mathrm{Ax}} \cup \mathrm{C}_{\text {Key }}\right)$ and $n \geq 1$. In this case, we have

$$
\begin{aligned}
f(p) & =c\left(f\left(p_{1}\right), \ldots, f\left(p_{n}\right)\right) \\
f(t) & =c\left(f\left(p_{1} \theta^{\prime}\right), \ldots, f\left(p_{n} \theta^{\prime}\right)\right)
\end{aligned}
$$

Let $v_{j}=p_{j} \theta^{\prime}$ for $j \in \widetilde{n}$. We prove this case by the following calculation:

$$
\begin{aligned}
f(t \theta) & =g\left(f\left(v_{1} \theta\right), \ldots, f\left(v_{n} \theta\right)\right) \\
& =g\left(f\left(v_{1}\right) f(\theta), \ldots, f\left(v_{n}\right) f(\theta)\right) \quad \text { by IH } \\
& =g\left(f\left(v_{1}\right), \ldots, f\left(v_{n}\right)\right) f(\theta) \\
& =f(t) f(\theta)
\end{aligned}
$$

This completes the proof of the theorem. 


\section{Deducibility preservation}

Notation. For the sake of a lighter notation, we will omit set braces in intruder derivations and write, e.g., $T, t \vdash_{E} u$ instead of $T \cup\{t\} \vdash_{E} u$ for a set of terms $T$ and individual terms $t$ and $u$. We also write $T \vdash_{E} U$ for a set of terms $U$ to mean that all terms in $U$ are derivable from those in $T$. In the remainder of this section, we assume that $F_{f}$ is $R, A x$-closed.

\section{D.1 Preservation results for equality and reduction}

We assume a standard derivation system for equational logic with an axiom rule including substitution as well as reflexivity, transitivity, and congruence rules.

Proposition 3 (Ax-equality preservation). Let $t, u \in \mathcal{T}$. Then $t={ }_{A x} u$ implies $f(t)={ }_{A x} f(u)$.

Proof. By induction on the derivation of $t={ }_{A x} u$. The cases are:

- Axiom: In this case, there are a pair $\left\{s_{1}, s_{2}\right\} \in A x$ and a substitution $\sigma$ such that $t=s_{1} \sigma$ and $u=s_{2} \sigma$. Since $F_{f}$ is $R, A x$-closed, we know that $s_{1}, s_{2} \in \operatorname{udom}\left(F_{f}\right)$. Moreover, by point $(\mathrm{v})$ in Definition 5 , we know that $f$ is homomorphic for $c t\left(s_{1}\right) \cup \operatorname{ct}\left(s_{2}\right)$. Therefore, we derive

$$
\begin{aligned}
& f(t)=f\left(s_{1}\right) f(\sigma)=s_{1} f(\sigma) \\
& f(u)=f\left(s_{2}\right) f(\sigma)=s_{2} f(\sigma)
\end{aligned}
$$

Thus by rule Axiom, we obtain that $s_{1} f(\sigma)={ }_{A x} s_{2} f(\sigma)$. Therefore, we have $f(t)={ }_{A x} f(u)$.

- Reflexivity: This case holds trivially, since we have $t=u$ (syntactic identity).

- Transitivity: Suppose there is a term $t^{\prime}$ such that $t={ }_{A x} t^{\prime}$ and $t^{\prime}={ }_{A x} u$. By the induction hypothesis, we have $f(t)={ }_{A x} f\left(t^{\prime}\right)$ and $f\left(t^{\prime}\right)={ }_{A x} f(u)$. Hence, $f(t)={ }_{A x} f(u)$ as required.

- Congruence: Suppose that $t=c\left(t_{1}, \ldots, t_{n}\right)$ and $u=c\left(u_{1}, \ldots, u_{n}\right)$ for some $c \in \Sigma^{n}$ and, for all $i \in \widetilde{n}$, terms $t_{i}$ and $u_{i}$ such that

$$
t_{i}={ }_{A x} u_{i}
$$

Since $F_{f}$ is $R, A x$-closed, we know that $t$ and $u$ match the same clause $f(p)=$ $q$ in $E_{f}^{+}$. Hence there are substitutions $\theta, \theta^{\prime}$ such that $t=p \theta$ and $u=p \theta^{\prime}$. We consider different shapes of $p$.

- $p=c\left(p_{1}, \ldots, p_{n}\right)$ for $c \in \Sigma^{n} \backslash\left(\mathrm{C}_{\mathrm{Ax}} \cup \mathrm{C}_{\mathrm{Key}}\right)$ and $n \geq 1$. In this case, we have

$$
f(p)=\left\langle e_{1}, \ldots, e_{d}\right\rangle
$$

for some $d>0$ for all $i \in \widetilde{d}, e_{i}$ is one of the following forms:

1. $e_{i}=c\left(\widehat{f}\left(\overline{q_{1}}\right), \ldots, \widehat{f}\left(\overline{q_{n}}\right)\right)$ such that

$$
\begin{aligned}
& \operatorname{set}\left(\overline{q_{j}}\right) \subseteq \operatorname{split}\left(p_{j}\right) \text { for all } j \in \widetilde{n}, c \neq\langle\cdot, \cdot\rangle, \text { and } \\
& c \in \mathrm{C}_{\mathrm{R}} \Rightarrow \overline{q_{n}}=\left[p_{n}\right]
\end{aligned}
$$


2. $e_{i}=f(q)$ such that $q \in \operatorname{split}\left(p_{i}\right)$ for some $j \in \widetilde{n}$. and it holds that $\forall j \in p p(c)$. split $\left(p_{j}\right) \subseteq Q(j)$ where

$$
\begin{gathered}
Q(j)=\bigcup\left\{\operatorname{set}\left(\overline{q_{j}}\right) \mid c\left(\widehat{f}\left(\overline{q_{1}}\right), \ldots, \widehat{f}\left(\overline{q_{n}}\right)\right) \in\left\{e_{1}, \ldots, e_{d}\right\}\right\} \\
\bigcup\left\{q \mid f(q) \in\left\{e_{1}, \ldots, e_{d}\right\}\right\}
\end{gathered}
$$

Hence, we have

$$
\begin{aligned}
f(t) & =\left\langle e_{1} \theta, \ldots, e_{d} \theta\right\rangle \\
f(u) & =\left\langle e_{1} \theta^{\prime}, \ldots, e_{d} \theta^{\prime}\right\rangle
\end{aligned}
$$

To see that $f(t)={ }_{A x} f(u)$, it is sufficient to show that $e_{i} \theta={ }_{A x} e_{i} \theta^{\prime}$ for all $i \in \widetilde{d}$. Let $i \in \widetilde{d}$. We distinguish two cases depending on the shape of $e_{i}$.

* If $e_{i}=c\left(\widehat{f}\left(\overline{q_{1}}\right), \ldots, \widehat{f}\left(\overline{q_{n}}\right)\right)$ then by $(6)$, we derive that there is a subderivation $e \theta={ }_{A x} e \theta^{\prime}$, for all $j \in \widetilde{d}$ and all $e \in \operatorname{set}\left(q_{j}\right)$. Hence, by induction hypothesis, we know that $f(e \theta)={ }_{A x} f\left(e \theta^{\prime}\right)$ for all $j \in \widetilde{d}$ and all $e \in \operatorname{set}\left(q_{j}\right)$. This implies $e_{i} \theta={ }_{A x} e_{i} \theta^{\prime}$ as desired.

* If $e_{i}=f(q)$ where $q \in \operatorname{split}\left(p_{j}\right)$ for some $j \in \widetilde{n}$, then by a similar reasoning as before, we derive that there is a sub-derivation $q \theta={ }_{A x} q \theta^{\prime}$. By induction hypothesis, we have $f(q \theta)={ }_{A x} f\left(q \theta^{\prime}\right)$ which implies $e_{i} \theta={ }_{A x} e_{i} \theta^{\prime}$ as desired.

- $p=c\left(p_{1}, \ldots, p_{n}\right)$ for $c \in \Sigma^{n} \cap\left(\mathrm{C}_{\mathrm{Ax}} \cup \mathrm{C}_{\mathrm{Key}}\right), n \geq 1$. Thus, we have

$$
\begin{aligned}
f(p) & =c\left(f\left(p_{1}\right), \ldots, f\left(p_{n}\right)\right), \\
f(t) & =c\left(f\left(t_{1}\right), \ldots, f\left(t_{n}\right)\right), \\
f(u) & =c\left(f\left(u_{1}\right), \ldots, f\left(u_{n}\right)\right) .
\end{aligned}
$$

By the induction hypothesis, we have $f\left(t_{i}\right)={ }_{A x} f\left(u_{i}\right)$ for all $i \in \widetilde{n}$. It follows that $f(t)={ }_{A x} f(u)$ as required.

This completes the proof of the lemma.

Proposition 4 (Reduction preservation). Let $t \in \operatorname{rdom}\left(F_{f}\right)$ be a term. Suppose $t \rightarrow_{R, A x} u$ for some term $u$. Then $f(t) \rightarrow_{R, A x} f(u)$.

Proof. Suppose $t \in \operatorname{rdom}\left(F_{f}\right)$ and $t \rightarrow_{R, A x} u$ for some term $u$. Then there are a position $p$ in $t$, a rewrite rule $l \rightarrow r \in R$, and substitution $\sigma$ such that $\left.t\right|_{p}={ }_{A x} l \sigma$ and $t \rightarrow_{R, A x} t[r \sigma]_{p}=u$. By Proposition 3, we have $f\left(\left.t\right|_{p}\right)={ }_{A x} f(l \sigma)$. Assuming without loss of generality that $\left.t\right|_{p}$ and $l$ have disjoint sets of variables, $\sigma$ is also a $R, A x$-unifier of $t_{p}$ and $l$. Therefore, since $t \in \operatorname{rdom}\left(F_{f}\right)$, we know from point (i) of Definition 9 that $f(l \sigma)=l f(\sigma)$ and from point (ii) that $f\left(\left.t\right|_{p}\right)=\left.f(t)\right|_{p}$. It follows that $\left.f(t)\right|_{p}={ }_{A x} l f(\sigma)$ and thus

$$
f(t) \rightarrow_{R, A x} f(t)[r f(\sigma)]_{p} .
$$

Since $r$ is either a variable of $l$ or a constant, we have $r f(\sigma)=f(r \sigma)$. Finally, by Definition 9(ii), we have $f(t)[f(r \sigma)]_{p}=f\left(t[r \sigma]_{p}\right)$. Hence, we have established $f(t) \rightarrow_{R, A x} f(u)$ as required. 
Lemma 11. Let $t \in \operatorname{rdom}\left(F_{f}\right)$ be a term and $p$ a position such that all strict subterms of $\left.t\right|_{p}$ are $R, A x$-normal. Suppose $t=_{A x} t[l \sigma]_{p} \rightarrow_{R, A x} t[r \sigma]_{p}=u$ for some rewrite rule $l \rightarrow r \in R$ and some substitution $\sigma$. Then $u \in \operatorname{rdom}\left(F_{f}\right)$.

Proof. Suppose that there is a rule $l^{\prime} \rightarrow r^{\prime}$, a position $p^{\prime}$ in $u$, and an $A x$-unifier $\sigma^{\prime}$ of $l^{\prime}$ and $\left.u\right|_{p^{\prime}}$. We need to show two points.

(i) $f$ is homomorphic for all $c \in c t\left(l^{\prime}\right)$,

(ii) $f$ is homomorphic for $\operatorname{top}\left(\left.u\right|_{p^{\prime \prime}}\right)$ and $\operatorname{top}\left(\left.u\right|_{p^{\prime \prime}}\right) \neq \operatorname{top}\left(l^{\prime \prime}\right)$ for all strict prefixes $p^{\prime \prime}$ of $p^{\prime}$ and rewrite rules $l^{\prime \prime} \rightarrow r^{\prime \prime} \in R$ such that $\operatorname{ct}\left(l^{\prime \prime}\right)$ is not a singleton.

Note that since all strict subterms of $t_{p}$ are $R, A x$-normal, so is $r \sigma$ by $R, A x$ coherence and the form of our rewrite rules. We distinguish two cases.

(a) $p^{\prime}$ is not a strict prefix of $p$. Since $p$ cannot be a prefix of $p^{\prime}$ (since $r \sigma$ is $R, A x$-normal) the redex $\left.u\right|_{p^{\prime}}$ is also present in $t$. Therefore, (i) and (ii) follow from the assumption that $t \in \operatorname{rdom}\left(F_{f}\right)$.

(b) $p^{\prime}$ is a strict prefix of $p$. Then the redex $\left.u\right|_{p^{\prime}}$ is a new one that was created by the reduction $t \rightarrow_{R, A x} u$. Point (ii) immediately follows from $t \in \operatorname{rdom}\left(F_{f}\right)$, since $p^{\prime}$ is a strict prefix of $p$ and thus $\operatorname{top}\left(\left.u\right|_{p^{\prime \prime}}\right)=\operatorname{top}\left(t_{p^{\prime \prime}}\right)$ for all prefixes $p^{\prime \prime}$ of $p^{\prime}$. In particular, we have that $f$ is homomorphic for $\operatorname{top}\left(\left.u\right|_{p^{\prime \prime}}\right)$ and top $\left(\left.u\right|_{p^{\prime \prime}}\right) \neq \operatorname{top}\left(l^{\prime \prime}\right)$ for strict prefixes $p^{\prime \prime}$ of $p^{\prime}$ and rewrite rules $l^{\prime \prime} \rightarrow r^{\prime \prime} \in$ $R$ such that $c t\left(l^{\prime \prime}\right)$ is not a singleton. It remains to show point (i). From $\left.u\right|_{p^{\prime}} \sigma^{\prime}={ }_{A x} l^{\prime} \sigma^{\prime}$ and the well-formedness of the equational theory, we derive that $\operatorname{top}\left(l^{\prime}\right)=\operatorname{top}\left(\left.u\right|_{p^{\prime}}\right)$. Since $p^{\prime}$ is a prefix of $p$ and $t \in \operatorname{rdom}\left(F_{f}\right)$, we derive that $c t\left(l^{\prime}\right)$ is singleton. Therefore, point (i) follows from the fact that $f$ is homomorphic for top $\left(\left.u\right|_{p^{\prime}}\right)$.

This completes the proof of the lemma.

Lemma 12. Let $t \in \operatorname{rdom}\left(F_{f}\right)$. Then $f(t)={ }_{E} f\left(t \downarrow_{R, A x}\right)$.

Proof. Suppose that $t \rightarrow_{R, A x}^{*} u={ }_{A x} t \downarrow_{R, A x}$. By $R, A x$-convergence, we can assume without loss of generality that the rewriting in the sequence $t \rightarrow_{R, A x}^{*} u$ is done inside out, i.e., all strict subterms of the redexes in the sequence are $R, A x$-normal. Hence, using Lemma 11 and Proposition 4 in a routine induction on the length of the rewriting sequence, we can show that $f(t) \rightarrow_{R, A x}^{*} f(u)$. Moreover, we have $f(u)={ }_{A x} f\left(t \downarrow_{R, A x}\right)$ by Proposition 3. Hence, we obtain $f(t)={ }_{E} f\left(t \downarrow_{R, A x}\right)$ as required.

Theorem (Equality preservation; Justification of Theorem 2). Suppose $t, u \in \operatorname{rdom}\left(F_{f}\right)$. Then $t={ }_{E} u$ implies $f(t)={ }_{E} f(u)$.

Proof. Suppose $t=_{E} u$. Then $t \downarrow_{R, A x}=_{A x} u \downarrow_{R, A x}$. By Proposition 3, we have $f\left(t \downarrow_{R, A x}\right)={ }_{A x} f\left(u \downarrow_{R, A x}\right)$. From Lemma 12 we have $f(t)=_{E} f\left(t \downarrow_{R, A x}\right)$ and $f(u)={ }_{E} f\left(u \downarrow_{R, A x}\right)$. Hence, $f(t)={ }_{E} f(u)$ as required. 


\section{D.2 Preservation results for deducibility}

Theorem (Deducibility preservation; Justification of Theorem 3). Let $T \cup\{t\} \subseteq \mathcal{N}$ be a set of ground network messages such that $\mathcal{C} \subseteq T$ and $T$ is $R, A x$-normal. Then $T \vdash_{E} t$ implies $f(T) \vdash_{E} f\left(t \downarrow_{R, A x}\right)$.

Proof. By induction on the derivation $D$ of $T \vdash_{E} t$, depending on the last rule that has been applied in this derivation.

- Ax: In this case, we have $t \in T$. Therefore $f(t) \in f(T)$ and thus $f(T) \vdash_{E} f(t)$. Moreover, since $T$ is $R, A x$-normal, so is $t$. Thus by Proposition 3, we have $f(t)={ }_{A x} f\left(t \downarrow_{R, A x}\right)$. Hence, we obtain $f(T) \vdash_{E} f\left(t \downarrow_{R, A x}\right)$ as required.

- Eq: We have $T \vdash_{E} t^{\prime}$ and $t^{\prime}=_{E} t$. By induction hypothesis, we have $f(T) \vdash_{E}$ $f\left(t^{\prime} \downarrow_{R, A x}\right)$. Moreover, since $t^{\prime}=_{E} t$, we derive $t^{\prime} \downarrow_{R, A x}={ }_{A x} t \downarrow_{R, A x}$. By Proposition 3, we have $f\left(t^{\prime} \downarrow_{R, A x}\right)={ }_{A x} f\left(t \downarrow_{R, A x}\right)$. It follows that $f(T) \vdash_{E}$ $f\left(t \downarrow_{R, A x}\right)$ as required.

- Comp: In this case, $t=g\left(u_{1}, \ldots, u_{m}\right)$ for $g \in \Sigma^{m}$ and the rule's premises are $T \vdash_{E} u_{i}$ for $i \in \widetilde{m}$. The induction hypotheses are $f(T) \vdash_{E} f\left(u_{i} \downarrow_{R, A x}\right)$ for $i \in \widetilde{m}$. Note that $t \downarrow_{R, A x}=\left(g\left(u_{1} \downarrow_{R, A x}, \ldots, u_{m} \downarrow_{R, A x}\right)\right) \downarrow_{R, A x}$. Hence without loss of generality, we can assume that $u_{i}$ are $R, A x$-normal for all $i \in \widetilde{m}$. We distinguish two cases depending on whether $t$ is $R, A x$-normal or not.

Case 1: $t$ is not $R, A x$-normal. Since $u_{i}$ are $R, A x$-normal for all $i \in \widetilde{m}$, some rewrite rule $l \rightarrow r \in R$ can be applied at the root position of $t$, i.e., there is a substitution $\sigma$ such that $t={ }_{A x} l \sigma$. This rule is of one of the following types.

- R4: In this case, we have $t \downarrow_{R, A x}=a$ for some constant $a$. Hence $f(t)=$ $f(a)=a$. Since $\mathcal{C} \subseteq T$ and $\mathcal{C}=f(\mathcal{C})$, we have $f(T) \vdash_{E} f\left(t \downarrow_{R, A x}\right)$.

- R3: We have $t \downarrow_{R, A x}={ }_{A x} u_{j}$ for some $j \in \widetilde{m}$. By Proposition 3, we have $f\left(t \downarrow_{R, A x}\right)={ }_{A x} f\left(u_{j}\right)$ which implies $f(T) \vdash_{E} f\left(t \downarrow_{R, A x}\right)$ by the induction hypothesis.

- R1: We have $m=2$ and

$$
\begin{aligned}
& l=g\left(c\left(x_{1}, \ldots, x_{n-1}, t^{\prime}\right), u^{\prime}\right) \rightarrow x_{j}, \\
& r=x_{j} \text { for some } j, 1 \leq j \leq n-1,
\end{aligned}
$$

where $x_{1}, \ldots, x_{n-1}$ are variables and $t^{\prime}, u^{\prime}$ are terms.

Let $t_{i}=x_{i} \sigma$ for all $i, 1 \leq i \leq n-1$ and $t_{n}=t^{\prime} \sigma$. Then we have

$$
\begin{aligned}
& u_{1}=c\left(t_{1}, \ldots, t_{n-1}, t_{n}\right), \\
& u_{2}=u^{\prime} \sigma .
\end{aligned}
$$

Moreover, since $u_{1}$ is $R, A x$-normal, so is $t_{j}$. Therefore, we have $t \downarrow_{R, A x}={ }_{A x}$ $t_{j}$. By Proposition 3, we know that $f\left(t \downarrow_{R, A x}\right)={ }_{A x} f\left(t_{j}\right)$. Therefore, to see that $f(T) \vdash_{E} f\left(t \downarrow_{R, A x}\right)$, it is sufficient to show that $f(T) \vdash_{E} f\left(t_{j}\right)$. From the induction hypothesis, we know that $f(T) \vdash_{E} f\left(u_{1}\right)$. By Lemma 6 , we have

$$
f\left(u_{1}\right)=\left\langle w_{1}, \ldots, w_{d}\right\rangle
$$

for some $d>0$ for all $i \in \widetilde{d}, w_{i}$ is one of the following forms: 
1. $w_{i}=c\left(\widehat{f}\left(\overline{v_{1}}\right), \ldots, \widehat{f}\left(\overline{v_{n}}\right)\right)$ such that

$$
\begin{aligned}
& \operatorname{split}\left(\overline{v_{k}}\right) \subseteq \operatorname{split}\left(t_{k}\right) \text { for all } k \in \widetilde{n}, c \neq\langle\cdot, \cdot\rangle \text {, and } \\
& c \in \mathrm{C}_{\mathrm{R}} \Rightarrow \overline{v_{n}}=\left[t_{n}\right],
\end{aligned}
$$

2. $w_{i}=f(v)$ where $\operatorname{split}(v) \subseteq \operatorname{split}\left(t_{k}\right)$ for some $k \in \widetilde{n}$

and it holds that

$$
\begin{aligned}
& \forall k \in p p(c) . \operatorname{split}\left(t_{k}\right) \subseteq \operatorname{split}(P(k)) \\
& \forall k \in \widetilde{n} . P(k) \subseteq \operatorname{subs}\left(u_{1}\right) \backslash\left\{u_{1}\right\}
\end{aligned}
$$

where

$$
\begin{gathered}
P(k)=\bigcup\left\{\operatorname{set}\left(\overline{v_{k}}\right) \mid c\left(\widehat{f}\left(\overline{v_{1}}\right), \ldots, \widehat{f}\left(\overline{v_{n}}\right)\right) \in\left\{w_{1}, \ldots, w_{d}\right\}\right\} \\
\bigcup\left\{v \mid f(v) \in\left\{w_{1}, \ldots, w_{d}\right\}\right\}
\end{gathered}
$$

By (7) and Lemma 10, it is sufficient to establish $f(T) \vdash_{E} f(e)$ for all $e \in P(j)$ in order to conclude the desired $f(T) \vdash_{E} f\left(t_{j}\right)$.

Let $e \in P(j)$. Note that there is $k \in \widetilde{d}$ such that one of the following holds.

$$
\begin{aligned}
& w_{k}=c\left(\widehat{f}\left(\overline{v_{1}}\right), \ldots, \widehat{f}\left(\overline{v_{n}}\right)\right) \text { and } e \in \operatorname{set}\left(\overline{v_{j}}\right) \\
& w_{k}=f(v) \text { and } e=v
\end{aligned}
$$

Clearly, we can derive $f(T) \vdash_{E} w_{k}$ from $f(T) \vdash_{E} f\left(u_{1}\right)$ by projections. We have

$$
f(T) \vdash_{E} c\left(\widehat{f}\left(\overline{v_{1}}\right), \ldots, \widehat{f}\left(\overline{v_{n}}\right)\right)
$$

In the second case, we immediately obtain $f(T) \vdash_{E} f(e)$. In the first case, we know that $\overline{v_{n}}=\left[t_{n}\right]$ by Lemma 6 . Hence, we have

$$
\widehat{f}\left(\overline{v_{n}}\right)=f\left(t_{n}\right)=f\left(t^{\prime} \sigma\right)
$$

We also have $f\left(u_{2}\right)=f\left(u^{\prime} \sigma\right)$. Note that $c t\left(t^{\prime}\right) \cup c t\left(u^{\prime}\right) \subseteq \mathrm{C}_{\text {Key }}$. Hence, by Definition 5, we know that $f$ is homomorphic for all $c \in \operatorname{ct}\left(t^{\prime}\right) \cup \operatorname{ct}\left(u^{\prime}\right)$. As before, we obtain

$$
\begin{gathered}
f\left(t_{n}\right)=t^{\prime} f(\sigma) \\
f\left(u_{2}\right)=u^{\prime} f(\sigma)
\end{gathered}
$$

Moreover, by induction hypothesis, we have $f(T) \vdash_{E} f\left(u_{2}\right)$. Together with (8), (9), and (10), we derive

$$
\left.f(T) \vdash_{E} g\left(c\left(\widehat{f}\left(\overline{v_{1}}\right), \ldots, \widehat{f}\left(\overline{v_{n-1}}\right), t^{\prime} f(\sigma)\right), u^{\prime} f(\sigma)\right)\right)
$$

Since $\left(\operatorname{vars}\left(t^{\prime}\right) \cup \operatorname{vars}\left(u^{\prime}\right)\right) \cap \bigcup_{k=1}^{n-1} x_{k}=\emptyset$ and $x_{p} \neq x_{q}$ for all $p, q$ such that $1 \leq p \neq q \leq n-1$, we can define a substitution $\theta$ as follows.

$$
\begin{aligned}
& x_{q} \theta=\widehat{f}\left(\overline{v_{q}}\right) \text { for } 1 \leq q \leq n-1, \\
& x \theta=x f(\sigma) \text { for all } x \in \operatorname{vars}\left(u^{\prime}\right) \cup \operatorname{vars}\left(t^{\prime}\right) .
\end{aligned}
$$

Then we have $\left.g\left(c\left(\widehat{f}\left(\overline{v_{1}}\right), \ldots, \widehat{f}\left(\overline{v_{n-1}}\right), t^{\prime} f(\sigma)\right), u^{\prime} f(\sigma)\right)\right)=l \theta$. Thus, we derive $\left.g\left(c\left(\widehat{f}\left(\overline{v_{1}}\right), \ldots, \widehat{f}\left(\overline{v_{n-1}}\right), t^{\prime} f(\sigma)\right), u^{\prime} f(\sigma)\right)\right) \rightarrow_{R, A x} \widehat{f}\left(\overline{v_{j}}\right)$. This implies $f(T) \vdash_{E} \widehat{f}\left(\overline{v_{j}}\right)$. Together with $e \in \operatorname{set}\left(\overline{v_{j}}\right)$ and Lemma 10, we derive that $f(T) \vdash_{E} f(e)$.

- R2: This case is treated similarly as the case for R1. 
Case 2: $t$ is $R, A x$-normal. By Proposition 3, we have $f(t)={ }_{A x} f\left(t \downarrow_{R, A x}\right)$. Hence, it is sufficient to show that $f(T) \vdash_{E} f(t)$. We do case analysis on $g$.

- If $g \in \Sigma^{n} \backslash\left(\mathrm{C}_{\mathrm{Ax}} \cup \mathrm{C}_{\mathrm{Key}}\right)$ then by Lemma 6 , we know that

$$
f(t)=\left\langle w_{1}, \ldots, w_{d}\right\rangle
$$

for some $d>0$ and for all $i \in \widetilde{d}, w_{i}$ is one of the following forms:

1. $w_{i}=g\left(\widehat{f}\left(\overline{v_{1}}\right), \ldots, \widehat{f}\left(\overline{v_{m}}\right)\right)$ such that

$$
\begin{aligned}
& \operatorname{split}\left(\overline{v_{k}}\right) \subseteq \operatorname{split}\left(u_{k}\right) \text { for all } k \in \widetilde{m}, g \neq\langle\cdot, \cdot\rangle, \\
& \text { and } g \in \mathrm{C}_{\mathrm{R}} \Rightarrow \overline{v_{m}}=\left[u_{m}\right]
\end{aligned}
$$

2. $w_{i}=f(v)$ such that $\operatorname{split}(v) \subseteq \operatorname{split}\left(u_{k}\right)$ for some $k \in \widetilde{m}$ and it holds that

$$
\begin{aligned}
& \forall k \in p p(g) . \operatorname{split}\left(u_{k}\right) \subseteq \operatorname{split}(P(k)) \\
& \forall k \in \widetilde{m} . P(k) \subseteq \operatorname{subs}(t) \backslash\{t\}
\end{aligned}
$$

where

$$
\begin{gathered}
P(k)=\bigcup\left\{\operatorname{set}\left(\overline{v_{k}}\right) \mid g\left(\widehat{f}\left(\overline{v_{1}}\right), \ldots, \widehat{f}\left(\overline{v_{m}}\right)\right) \in\left\{w_{1}, \ldots, w_{d}\right\}\right\} \\
\bigcup\left\{v \mid f(v) \in\left\{w_{1}, \ldots, w_{d}\right\}\right\}
\end{gathered}
$$

To see that $f(T) \vdash_{E} f(t)$, it is sufficient to show that $f(T) \vdash_{E} w_{i}$ for all $i \in \widetilde{d}$. Let $i \in \widetilde{d}$. We do a case distinction on the shape of $w_{i}$.

* If $w_{i}=c\left(\widehat{f}\left(\overline{v_{1}}\right), \ldots, \widehat{f}\left(\overline{v_{m}}\right)\right)$ then since $\operatorname{split}\left(\overline{v_{j}}\right) \subseteq \operatorname{split}\left(u_{j}\right)$ for all $j \in \widetilde{m}$, by Lemma 10, we derive that $f\left(u_{j} \vdash_{E} \widehat{f}\left(\overline{v_{j}}\right)\right.$ for all $j \in \widetilde{m}$. Moreover, by induction hypothesis, we have $f(T) \vdash_{E} f\left(u_{j}\right)$ for all $j \in \widetilde{m}$. Therefore, we derive that $f(T) \vdash_{E} \widehat{f}\left(\overline{v_{j}}\right)$ for all $j \in \widetilde{m}$. This implies $f(T) \vdash_{E} w_{i}$ as desired.

* If $w_{i}=f(v)$ for some term $v$ such that $\operatorname{split}(v) \subseteq \operatorname{split}\left(u_{k}\right)$ for some $k \in \widetilde{m}$. By a similar reasoning as above, we conclude that $f(T) \vdash_{E} w_{i}$.

- For the remaining cases, we have $f(t)=g\left(f\left(u_{1}\right), \ldots, f\left(u_{m}\right)\right)$. Then $f(T) \vdash_{E} f(t)$ follows immediately from the induction hypothesis.

This completes the proof of the theorem.

Lemma 13. Let $\theta$ be a well-typed $R, A x$-normal ground substitution and $t \in$ $\operatorname{rdom}\left(F_{f}\right)$. Then $t \theta \in \operatorname{rdom}\left(F_{f}\right)$.

Proof. Suppose $p$ is a non-variable position in $t \theta$ and $l \rightarrow r \in R$ is a rewrite rule such that there exists a well-typed $A x$-unifier $\sigma$ of $\left.t \theta\right|_{p}$ and $l$, i.e., $\left.t \theta\right|_{p} \sigma={ }_{A x} l \sigma$. We need to show two points.

(i) $f$ is homomorphic for all $c \in c t(l)$,

(ii) $f$ is homomorphic for $\operatorname{top}\left(\left.t \theta\right|_{p^{\prime}}\right)$ and $t o p\left(\left.t \theta\right|_{p^{\prime}}\right) \neq t o p\left(l^{\prime}\right)$ for all strict prefixes $p^{\prime}$ of $p$ and rewrite rules $l^{\prime} \rightarrow r^{\prime} \in R$ such that $c t\left(l^{\prime}\right)$ is not a singleton. 
Since $\theta$ is ground, we have $\left.t \theta\right|_{p} \sigma=\left.t \theta\right|_{p}$. Since $\theta$ is $R, A x$-normal, we derive that $p$ is a position in $t$ and $\left.t\right|_{p}$ is not a variable. Thus, we have $\left.t \theta\right|_{p}=\left.t\right|_{p} \theta$. Hence, $\left.t\right|_{p} \theta={ }_{A x} l \sigma$. Assuming without loss of generality that $\left.t\right|_{p}$ and $l$ have disjoint sets of variables, $\theta \cup \sigma$ is an $A x$-unifier of $\left.t\right|_{p}$ and $l$. Since $\left.t\right|_{p}$ is not a variable, we have $\operatorname{top}\left(\left.t\right|_{p}\right)=\operatorname{top}\left(\left.t\right|_{p} \theta\right)=\operatorname{top}\left(\left.t \theta\right|_{p}\right)$. Therefore, we derive (i) and (ii) from Definition 9 and $t \in \operatorname{rdom}\left(F_{f}\right)$. Hence, $t \theta \in \operatorname{rdom}\left(F_{f}\right)$ as required.

We are now ready to establish deducibility preservation for open terms. This result is crucial to for reachability preservation.

Proposition 5 (Deducibility preservation for open terms). Let $\sigma$ be a well-typed R,Ax-normal ground substitution, $T$ be a set of terms, and $u$ be term such that

(i) $f\left(I K_{0}\right) \subseteq I K_{0}^{\prime}$,

(ii) $T \cup\{u\} \subseteq$ udom $\left(F_{f}\right) \cap \operatorname{rdom}\left(F_{f}\right)$.

Then $T \sigma, I K_{0} \vdash_{E} u \sigma$ implies $f(T) f(\sigma), I K_{0}^{\prime} \vdash_{E} f(u) f(\sigma)$.

Proof. We have

$$
\begin{aligned}
& T \sigma, I K_{0} \quad \vdash_{E} u \sigma \quad \text { by assumption } \\
& \Rightarrow(T \sigma) \downarrow_{R, A x}, I K_{0} \quad \vdash_{E}(u \sigma) \downarrow_{R, A x} \quad \text { by rule Eq } \\
& \Rightarrow f\left((T \sigma) \downarrow_{R, A x}\right), f\left(I K_{0}\right) \vdash_{E} f\left((u \sigma) \downarrow_{R, A x}\right) \quad \text { by Theorem } 3 \\
& \Rightarrow f\left((T \sigma) \downarrow_{R, A x}\right), I K_{0}^{\prime} \quad \vdash_{E} f\left((u \sigma) \downarrow_{R, A x}\right) \quad \text { by assumption (i) } \\
& \Rightarrow f(T \sigma), I K_{0}^{\prime} \quad \vdash_{E} f(u \sigma) \quad \text { by Lemma 13, Theorem } 2 \text { and Eq } \\
& \Rightarrow f(T) f(\sigma), I K_{0}^{\prime} \quad \vdash_{E} f(u) f(\sigma) \quad \text { by Theorem } 1
\end{aligned}
$$

This completes the proof of the proposition. 


\section{E Soundness of typed abstractions (Section 3.2)}

\section{E.1 Reachability preservation}

Using the deducibility preservation for open terms, we show that each reachable state in the original protocol can be simulated by one in the abstracted protocol.

Theorem 5 (Reachability preservation). Suppose that

(i) $f\left(I K_{0}\right) \subseteq I K_{0}^{\prime}$,

(ii) $F_{f}$ is $R$, Ax-closed,

(iii) $\mathcal{M}_{P} \subseteq \operatorname{udom}\left(F_{f}\right) \cap \operatorname{rdom}\left(F_{f}\right)$.

Let $(\mathrm{tr}, \mathrm{th}, \sigma)$ be a reachable state of $P$ such that $\sigma$ is $R, A x$-normal. Then $(f(t r), f(t h), f(\sigma))$ is a reachable state of $f(P)$.

Proof. Note that $f(\sigma)$ is a well-typed ground substitution by Lemma 7 . We now show that $(f(t r), f(t h), f(\sigma))$ is reachable in $f(P)$ by induction on the number $n$ of transitions leading to a state $(t r, t h, \sigma)$.

- Base case $(n=0)$ : For all $i \in \operatorname{dom}(t h)$, there exists $R \in \operatorname{dom}(P)$ such that $t h(i)=(R, P(R))$. Hence we have

$$
f(t h)(i)=(R, f(P(R)))=(R, f(P)(R))
$$

Since $(\epsilon, t h, \sigma)$ is reachable, for all $v \in \operatorname{dom}(P)$ and $i \in T I D$ we have $v^{\# i} \sigma \in$ $\mathcal{A}$. Moreover, we have $v^{\# i} f(\sigma)=v^{\# i} \sigma$, we also have

$$
v^{\# i} f(\sigma) \in \mathcal{A}
$$

By $(11),(12)$ and $f(\epsilon)=\epsilon$, it is obvious that $(f(\epsilon), f(t h), f(\sigma))$ is reachable in $f(P)$.

- Inductive case $(n=k+1)$ : Suppose $\left(t r^{\prime}, t h^{\prime}, \sigma\right)$ is reachable in $k$ steps and there is a transition $\left(t r^{\prime}, t h^{\prime}, \sigma\right) \rightarrow(t r, t h, \sigma)$. By induction hypothesis, we have

$$
\left(f\left(t r^{\prime}\right), f\left(t h^{\prime}\right), f(\sigma)\right) \text { is reachable in } f(P)
$$

We consider two cases according to the rule $r$ that has been applied in step $k+1$.

- If $r=S E N D$ then there exists $i \in T I D$ and $R \in \operatorname{dom}(P)$ such that

$$
\begin{aligned}
t h^{\prime}(i) & =(R, \operatorname{snd}(p t) . t l) \\
t r & =t r^{\prime} \cdot(i, \operatorname{snd}(p t)) \\
t h & =t h^{\prime}[i \mapsto(R, t l)]
\end{aligned}
$$

By (14) we have

$$
\begin{aligned}
& f(t r)=f\left(t r^{\prime}\right) \cdot(i, \operatorname{snd}(f(p t))) \\
& f(t h)=f\left(t h^{\prime}\right)[i \mapsto(R, f(t l))]
\end{aligned}
$$


By (14) we have

$$
f\left(t h^{\prime}\right)(i)=(R, \operatorname{snd}(f(p t)) \cdot f(t l))
$$

By (16), (14), (15) and rule SEND, we have

$$
\left(f\left(t r^{\prime}\right), f\left(t h^{\prime}\right), f(\sigma)\right) \rightarrow(f(t r), f(t h), f(\sigma))
$$

Together with (13) this implies that $(f(t r), f(t h), f(\sigma))$ is reachable in $f(P)$.

- If $r=R E C V$ then there exists $i \in T I D$ and $R \in \operatorname{dom}(P)$ such that

$$
\begin{aligned}
& t h^{\prime}(i)=(R, \operatorname{rcv}(u) \cdot t l) \\
& I K\left(t r^{\prime}\right) \sigma, I K_{0} \vdash_{E} u \sigma
\end{aligned}
$$

and

$$
\begin{aligned}
& t r=t r^{\prime} \cdot(i, \operatorname{rcv}(u)) \\
& t h=t h^{\prime}[i \mapsto(R, t l)]
\end{aligned}
$$

By (17) and (18) we have

$$
\begin{aligned}
& f(t r)=f\left(t r^{\prime}\right) \cdot(i, \operatorname{rcv}(f(u))) \\
& f(t h)=f\left(t h^{\prime}\right)[i \mapsto(R, f(t l))]
\end{aligned}
$$

To justify $\left(f\left(t r^{\prime}\right), f\left(t h^{\prime}\right), f(\sigma)\right) \rightarrow(f(t r), f(t h), f(\sigma))$, it is sufficient to establish the following two premises of rule $R E C V$ :

1. $f\left(t h^{\prime}\right)(i)=(R, \operatorname{rcv}(f(u)) \cdot f(t l))$, which follows from (17), and

2. $I K\left(f\left(t r^{\prime}\right)\right) f(\sigma), I K_{0}^{\prime} \vdash_{E} f(u) f(\sigma)$. This follows from (17), Proposition 5 , and the fact that $f\left(I K\left(t r^{\prime}\right)\right)=I K\left(f\left(t r^{\prime}\right)\right)$.

Together with (13) this implies that $(f(t r), f(t h), f(\sigma))$ is reachable in $f(P)$.

This completes the proof of the theorem.

\section{E.2 Soundness}

We show in the following lemma that whenever a protocol admits an attack then there is an $R, A x$-normal attack.

Lemma 14. Let $\phi \in \mathcal{L}_{P}$ and let $(t r, t h, \sigma)$ be a reachable state of $P$. Then the following holds.

(i) $\left(t r, t h, \sigma \downarrow_{R, A x}\right)$ is a reachable state in $P$, and

(ii) if $(t r, t h, \sigma) \not \models \phi$ then $\left(t r, t h, \sigma \downarrow_{R, A x}\right) \not \models \phi$.

Proof. Let $\sigma^{\prime}=\sigma \downarrow_{R, A x}$. Then $\sigma^{\prime}$ is well-typed. Next, we show reachability for $\sigma^{\prime}$, i.e., we need to show that $\left(t r, t h, \sigma^{\prime}\right)$ is reachable in $P$. We prove this by induction on the number $n$ of transitions leading to $(t r, t h, \sigma)$.

- Base case $(n=0)$ : Since $(\epsilon, t h, \sigma)$ is reachable, so is $\left(\epsilon, t h, \sigma^{\prime}\right)$. 
- Inductive case $(n=k+1)$ : Suppose $\left(t r^{\prime}, t h^{\prime}, \sigma\right)$ is reachable in $k$ steps and there is a transition $\left(t r^{\prime}, t h^{\prime}, \sigma\right) \rightarrow(t r, t h, \sigma)$. By induction hypothesis, we have

$$
\left(t r^{\prime}, t h^{\prime}, \sigma\right) \text { is reachable in } P \text {. }
$$

We consider two cases according to the rule $r$ that has been applied in step $k+1$.

- If $r=S E N D$ then it is obvious that $\left(t r^{\prime}, t h^{\prime}, \sigma^{\prime}\right) \rightarrow\left(t r, t h, \sigma^{\prime}\right)$. This by (19) yields that $\left(t r, t h, \sigma^{\prime}\right)$ is reachable in $P$.

- If $r=R E C V$ then there exists $i \in T I D$ and $R \in \operatorname{dom}(P)$ such that $t h^{\prime}(i)=(R, \operatorname{rcv}(u) \cdot t l), t r=t r^{\prime} \cdot(i, \operatorname{rcv}(u)), t h=t h^{\prime}[i \mapsto(R, t l)]$, and

$$
I K\left(t r^{\prime}\right) \sigma, I K_{0} \vdash_{E} u \sigma
$$

Hence, we have $I K\left(t r^{\prime}\right) \sigma^{\prime}, I K_{0} \vdash_{E} u \sigma^{\prime}$. Thus the reachability of $\left(t r, t h, \sigma^{\prime}\right)$ in $P$ follows immediately.

Finally, we show attack preservation for $\sigma^{\prime}$, i.e., we need to show that

$$
\forall \vartheta .(\operatorname{tr}, t h, \sigma, \vartheta) \not \models \phi \Rightarrow\left(t r, t h, \sigma^{\prime}, \vartheta\right) \not \models \phi
$$

We prove this by induction on the structure of $\phi$. Note that the base cases for the literals that do not depend on $\sigma$ are trivial. Hence, it is enough to consider the following cases.

$-\phi \equiv m=m^{\prime}$ or $\phi \equiv \neg\left(m=m^{\prime}\right)$.

$$
\begin{aligned}
& (t r, t h, \sigma, \vartheta) \vDash m=m^{\prime} \\
\Leftrightarrow & m \sigma={ }_{E} m^{\prime} \sigma \\
\Leftrightarrow & m \sigma^{\prime}={ }_{E} m^{\prime} \sigma^{\prime} \\
\Leftrightarrow & (t r, t h, \sigma \downarrow R, A x, \vartheta) \vDash m=m^{\prime}
\end{aligned}
$$

- $\phi \equiv \operatorname{honest}(i, R)$ or $\phi \equiv \neg$ honest $(i, R)$.

$$
\begin{aligned}
& (\operatorname{tr}, \operatorname{th}, \sigma, \vartheta) \vDash \operatorname{honest}(i, R) \\
\Leftrightarrow & R^{\vartheta(i)} \sigma \in \mathcal{A}_{H} \\
\Leftrightarrow & R^{\vartheta(i)} \sigma^{\prime} \in \mathcal{A}_{H} \\
\Leftrightarrow & \left(\operatorname{tr}, \operatorname{th}, \sigma^{\prime}, \vartheta\right) \vDash \operatorname{honest}(i, R)
\end{aligned}
$$

$-\phi \equiv \operatorname{secret}(m)$.

$$
\begin{aligned}
& (t r, t h, \sigma, \vartheta) \not \models \operatorname{secret}(m) \\
\Leftrightarrow & I K(t r) \sigma, I K_{0} \vdash_{E} m \sigma \\
\Rightarrow & I K(t r) \sigma^{\prime}, I K_{0} \vdash_{E} m \sigma^{\prime} \\
\Leftrightarrow & \left(\operatorname{tr}, t h, \sigma^{\prime}, \vartheta\right) \not \models \operatorname{secret}(m)
\end{aligned}
$$

The inductive cases are routines. 


\section{E.3 Soundness of typed abstractions}

Theorem (Soundness; Justification of Theorem 4). Suppose P, $\phi$, and $F_{f}$ satisfy

$-f\left(I K_{0}\right) \subseteq I K_{0}^{\prime}$

- $F_{f}$ is $R, A x$-closed,

$-\mathcal{M}_{P} \subseteq \operatorname{udom}\left(F_{f}\right) \cap \operatorname{rdom}\left(F_{f}\right)$, and

- $\phi$ is safe for $P$ and $f$.

Then, for all states $(t r$, th, $\sigma)$ reachable in $P$, we have

(i) $\left(f(t r), f(t h), f\left(\sigma \downarrow_{R, A x}\right)\right)$ is a reachable state of $f(P)$,

(ii) $($ tr, th, $\sigma) \not \models \phi$ implies $\left(f(t r), f(t h), f\left(\sigma \downarrow_{R, A x}\right)\right) \not \models f(\phi)$.

Proof. Let $(t r, t h, \sigma)$ be a reachable state of $P$. By Lemma 14, we know that (a) $\left(t r, t h, \sigma \downarrow_{R, A x}\right)$ is a reachable state of $P$, and (b) $(t r, t h, \sigma) \not \models \phi$ implies $\left(t r, t h, \sigma \downarrow_{R, A x}\right) \not \models \phi$. Let $\sigma^{\prime}=f\left(\sigma \downarrow_{R, A x}\right)$. Then point (i) follows from (a) and Theorem 5. Using (b) we reduce point (ii) to showing that $\left(t r, t h, \sigma \downarrow_{R, A x}\right) \not \models \phi$ implies $\left(f(t r), f(t h), \sigma^{\prime}\right) \not \models f(\phi)$, which we establish by proving the following generalized statement by induction on the structure of $\phi$ (which may now contain free thread-id variables).

$$
\forall \vartheta .\left(t r, t h, \sigma \downarrow_{R, A x}, \vartheta\right) \not \models \phi \Rightarrow\left(f(t r), f(t h), \sigma^{\prime}, \vartheta\right) \not \models f(\phi)
$$

Note that a formula is safe if and only if all its subformulas are safe. The literals form the base cases of the induction. We cover all atoms and their negations (except secret $(m)$ ) in a single equivalence-based argument, where the right-toleft direction covers the positive literal and the other direction the corresponding negative literal. We remark that $\left(\operatorname{tr}, t h, \sigma \downarrow_{R, A x}, \vartheta\right) \not \models A$ is equivalent to $\left(t r, t h, \sigma \downarrow_{R, A x}, \vartheta\right) \vDash \neg A$ for all atoms $A$ (but not for all formulas, since $\mathcal{L}_{P}$ is not closed under negation).

$$
\begin{aligned}
&-\phi \equiv i= j \text { or } \phi \equiv \neg(i=j) . \\
&(t r, t h, \sigma \downarrow R, A x, \vartheta) \vDash i=j \\
& \Leftrightarrow \vartheta(i)=\vartheta(j) \\
& \Leftrightarrow\left(f(t r), f(t h), \sigma^{\prime}, \vartheta\right) \vDash f(i=j) \\
&-\phi \equiv m=m^{\prime} .
\end{aligned}
$$

$$
\begin{aligned}
& \left(t r, t h, \sigma \downarrow_{R, A x}, \vartheta\right) \vDash m=m^{\prime} \\
& \Rightarrow m \sigma \downarrow_{R, A x}={ }_{E} m^{\prime} \sigma \downarrow_{R, A x} \\
& \Rightarrow f\left(m \sigma \downarrow_{R, A x}\right)={ }_{E} f\left(m^{\prime} \sigma \downarrow_{R, A x}\right) \\
& \text { by Theorem } 2 \\
& \Rightarrow f(m) f\left(\sigma \downarrow_{R, A x}\right)=f\left(m^{\prime}\right) f\left(\sigma \downarrow_{R, A x}\right) \\
& \Leftrightarrow f(m) \sigma^{\prime}=f\left(m^{\prime}\right) \sigma^{\prime} \\
& \Leftrightarrow\left(f(t r), f(t h), \sigma^{\prime}, \vartheta\right) \vDash f(m)=f\left(m^{\prime}\right)
\end{aligned}
$$


$-\phi \equiv \neg\left(m=m^{\prime}\right)$.

$$
\begin{aligned}
& \left(f(t r), f(t h), \sigma^{\prime}, \vartheta\right) \vDash f(m)=f\left(m^{\prime}\right) \\
& \Rightarrow f(m) \sigma^{\prime}={ }_{E} f\left(m^{\prime}\right) \sigma^{\prime} \\
& \Leftrightarrow f(m) f\left(\sigma \downarrow_{R, A x}\right)={ }_{E} f\left(m^{\prime}\right) f\left(\sigma \downarrow_{R, A x}\right) \\
& \Rightarrow f\left(m \sigma \downarrow_{R, A x}\right)={ }_{E} f\left(m^{\prime} \sigma \downarrow_{R, A x}\right) \\
& \Rightarrow m \sigma \downarrow_{R, A x}={ }_{E} m^{\prime} \sigma \downarrow_{R, A x} \\
& \Rightarrow\left(t r, t h, \sigma \downarrow_{R, A x}, \vartheta\right) \vDash m=m^{\prime}
\end{aligned}
$$

$-\phi \equiv \operatorname{role}(i, R)$ or $\phi \equiv \neg \operatorname{role}(i, R)$.

$$
\begin{aligned}
& (t r, t h, \sigma \downarrow R, A x, \vartheta) \vDash \operatorname{role}(i, R) \\
\Leftrightarrow & \exists \text { seq } \in \operatorname{Evt^{*}} . \operatorname{th}(\vartheta(i))=(R, \text { seq }) \\
\Leftrightarrow & \exists \operatorname{seq} \in E v t^{*} . f(t h)(\vartheta(i))=(R, f(s e q)) \\
\Leftrightarrow & \left(f(t r), f(t h), \sigma^{\prime}, \vartheta\right) \vDash \operatorname{role}(i, R)
\end{aligned}
$$

- $\phi \equiv$ honest $(i, R)$ or $\phi \equiv \neg$ honest $(i, R)$.

$$
\begin{aligned}
& \left(\operatorname{tr}, \text { th, } \sigma \downarrow_{R, A x}, \vartheta\right) \vDash \operatorname{honest}(i, R) \\
\Leftrightarrow & R^{\vartheta(i)} \sigma \downarrow_{R, A x} \in \mathcal{A}_{H} \\
\Leftrightarrow & R^{\vartheta(i)} f\left(\sigma \downarrow_{R, A x}\right) \in \mathcal{A}_{H} \\
\Leftrightarrow & R^{\vartheta(i)} \sigma^{\prime} \in \mathcal{A}_{H} \\
\Leftrightarrow & \left(f(t r), f(t h), \sigma^{\prime}, \vartheta\right) \vDash \operatorname{honest}(i, R)
\end{aligned}
$$

$f$ is the identity on $\mathcal{A}$ since $R^{\vartheta(i)} \sigma^{\prime}=R^{\vartheta(i)} f(\sigma \downarrow R, A x)$

$-\phi \equiv \operatorname{steps}(i, s(m))$ or $\phi \equiv \neg \operatorname{steps}(i, s(m))$, where $s \in\{\mathrm{snd}, \mathrm{rcv}\}$. We have

$$
\begin{array}{rlr} 
& \left(\operatorname{tr}, \operatorname{th}, \sigma \downarrow_{R, A x}, \vartheta\right) \vDash \operatorname{steps}(i, s(m)) & \\
\Leftrightarrow & \left(\vartheta(i), s\left(m^{\# \vartheta(i)}\right)\right) \in t r & \\
\Leftrightarrow & \left(\vartheta(i), s\left(f(m)^{\# \vartheta(i)}\right)\right) \in f(t r) & \text { justified below } \\
\Leftrightarrow & \left(f(t r), f(t h), \sigma^{\prime}, \vartheta\right) \vDash \operatorname{steps}(i, s(f(m))) &
\end{array}
$$

We show the second equivalence. The left-to-right implication holds, since $\phi$ is safe. For the inverse direction (covering the positive literal $\phi \equiv \operatorname{steps}(i, s(m))$ ), suppose that

$$
\left(\vartheta(i), s\left(f(m)^{\# \vartheta(i)}\right)\right) \in f(t r) .
$$

Then there exists $s\left(m^{\prime}\right) \in \operatorname{Evt}\left(\mathcal{M}_{P}\right)$ such that $\left(\vartheta(i), s\left(m^{\prime \# \vartheta(i)}\right)\right) \in \operatorname{tr}$ and $f\left(m^{\prime}\right)=f(m)$. Since $\phi$ is safe, this implies $m=m^{\prime}$ and hence $\left(\vartheta(i), s\left(m^{\# \vartheta(i)}\right)\right) \in$ tr.

- $\phi \equiv(i, s(m)) \prec\left(j, s^{\prime}\left(m^{\prime}\right)\right)$ or $\phi \equiv \neg\left((i, s(m)) \prec\left(j, s^{\prime}\left(m^{\prime}\right)\right)\right)$, where $s, s^{\prime} \in$ $\{\mathrm{snd}, \mathrm{rcv}\}$.

$$
\begin{aligned}
& \left(\operatorname{tr}, t h, \sigma \downarrow_{R, A x}, \vartheta\right) \vDash(i, s(m)) \prec\left(j, s^{\prime}\left(m^{\prime}\right)\right) \\
\Leftrightarrow & \left(\vartheta(i), s\left(m^{\# \vartheta(i)}\right)\right) \prec_{t r}\left(\vartheta(j), s^{\prime}\left(m^{\prime \# \vartheta(j)}\right)\right) \\
\Leftrightarrow & \left(\vartheta(i), s\left(f(m)^{\# \vartheta(i)}\right)\right) \prec_{f(t r)}\left(\vartheta(j), s^{\prime}\left(f\left(m^{\prime}\right)^{\# \vartheta(j)}\right)\right) \quad \text { justified below } \\
\Leftrightarrow & \left(f(t r), f(t h), \sigma^{\prime}, \vartheta\right) \vDash(i, s(f(m))) \prec\left(j, s^{\prime}\left(f\left(m^{\prime}\right)\right)\right)
\end{aligned}
$$

We show the second equivalence. Note that, the if-direction immediately follows, since $\phi$ is safe and $f$ is order-preserving for events. 
For the only-if direction (covering the case that $\left.\phi \equiv(i, s(m)) \prec\left(j, s^{\prime}\left(m^{\prime}\right)\right)\right)$, suppose $\left(\vartheta(i), s\left(f(m)^{\# \vartheta(i)}\right)\right) \prec_{f(t r)}\left(\vartheta(j), s^{\prime}\left(f\left(m^{\prime}\right)^{\# \vartheta(j)}\right)\right)$. Since $f$ is orderpreserving for events, there are $s(u), s^{\prime}\left(u^{\prime}\right) \in \operatorname{Evt}\left(\mathcal{M}_{P}\right)$ such that

$$
\left(\vartheta(i), s\left(u^{\# \vartheta(i)}\right)\right) \prec_{t r}\left(\vartheta(j), s^{\prime}\left(u^{\prime \# \vartheta(j)}\right)\right)
$$

with $f(u)=f(m)$ and $f\left(u^{\prime}\right)=f\left(m^{\prime}\right)$. Since $\phi$ is safe, we have $u=m$ and $u^{\prime}=m^{\prime}$, completing the proof of this direction.

$-\phi \equiv \operatorname{secret}(m)$.

$$
\begin{array}{rlr} 
& \left(\operatorname{tr}, t h, \sigma \downarrow_{R, A x}, \vartheta\right) \not \operatorname{secret}(m) & \\
\Leftrightarrow & I K(t r) \sigma \downarrow_{R, A x}, I K_{0} \vdash_{E} m \sigma \downarrow_{R, A x} & \\
\Rightarrow & f(I K(t r)) \sigma^{\prime}, I K_{0}^{\prime} \vdash_{E} f(m) \sigma^{\prime} & \text { by Proposition } 5 \\
\Rightarrow & I K(f(t r)) \sigma^{\prime}, I K_{0}^{\prime} \vdash_{E} f(m) \sigma^{\prime} & \text { since } f(I K(t r))=I K(f(t r))) \\
\Leftrightarrow & \left(f(t r), f(t h), \sigma^{\prime}, \vartheta\right) \not \models \operatorname{secret}(f(m)) &
\end{array}
$$

The inductive cases are routines. This concludes the proof of the theorem. 


\section{F Syntactic criteria for soundness conditions}

Conditions (i) in the definition of $R, A x$-closedness (Definition 8) and (iii) in the definition of safe formulas (Definition 10) are hard to check in practice, since they universally quantify over ground terms (the first condition) and welltyped $R, A x$-normal ground substitutions (the second one). We therefore propose syntactic criteria for verifying these conditions.

\section{F.1 Syntactic criterion for $\boldsymbol{R}, \boldsymbol{A x}$-closure}

Lemma 15. Suppose for all types $\tau \in \operatorname{subs}\left(\Pi\left(E_{f}^{+}\right)\right)$and all $\{s, t\} \in A x$ such that $\tau \downarrow \cap(\Gamma(s) \downarrow \cup \Gamma(t) \downarrow) \neq \emptyset$ implies $\Gamma(s) \preccurlyeq \tau$ and $\Gamma(t) \preccurlyeq \tau$. Then $F_{f}$ is $R$, Ax-closed.

Proof. Let $\tau \in \Pi\left(E_{f}^{+}\right)$and $u, v$ be $R, A x$-normal such that $u={ }_{A x} v, u: \tau_{u}$, $v: \tau_{v}$. Because of symmetry, it is sufficient to show that $\tau_{u} \preccurlyeq \tau$ implies $\tau_{v} \preccurlyeq \tau$. We show this by induction on the derivation $u={ }_{A x} v$ depending on the last rule that has been applied. Suppose $\tau_{u} \preccurlyeq \tau$.

- Reflexivity: In this case, we have $u=v$. Hence it is clear that $\tau_{v} \preccurlyeq \tau$.

- Axiom: In this case, there is a pair $\{s, t\} \in A x$ and a substitution $\sigma$ such that $u=s \sigma$ and $v=t \sigma$. Since $\tau_{u} \preccurlyeq \tau$, we have $\Gamma(s \sigma) \preccurlyeq \tau$. Moreover, we have $\Gamma(s \sigma) \preccurlyeq \Gamma(s)$. Hence, it holds that $\tau \downarrow \cap \Gamma(s) \downarrow \neq \emptyset$. By assumption, we have $\Gamma(t) \preccurlyeq \tau$. We also have $\Gamma(t \sigma) \preccurlyeq \Gamma(t)$. Therefore, we obtain that $\tau_{v} \preccurlyeq \tau$.

- Congruence: Suppose that $t=g\left(t_{1}, \ldots, t_{n}\right)$ for some $g \in \Sigma^{n}, n \geq 1$. We have $u=g\left(u_{1}, \ldots, u_{n}\right)$ and $t_{i}={ }_{A x} u_{i}$. Since $\tau_{t} \preccurlyeq \tau$, either $\tau=m s g$ or $\tau=g\left(\tau_{1}, \ldots, \tau_{n}\right)$ and $\Gamma\left(t_{i}\right) \preccurlyeq \tau_{i}$ for all $i \in \widetilde{n}$. In the first case, i.e., $\tau=m s g$, it is obvious that $\tau_{v} \preccurlyeq \tau$. In the latter case, by induction hypothesis, we know that $\Gamma\left(u_{i}\right) \preccurlyeq \tau_{i}$ for all $i \in \widetilde{n}$. This implies $\tau_{u} \preccurlyeq \tau$ as required.

- Transitivity: In this case, there is a term $w$ such that $t={ }_{A x} w$ and $w={ }_{A x} u$. By induction hypothesis, we have $\Gamma(w) \preccurlyeq \tau$ and $\tau_{u} \preccurlyeq \tau$ which concludes this case.

This completes the proof of the lemma.

We demonstrate an application of Lemma 15 in the following example.

Example 9. Consider the function specification $F_{1}=\left(f_{1}, E_{1}\right)$ in Example 6 . We recall the list of equations $E_{1}$ with variables $X_{3}: \gamma_{o}, Y_{3}$ : nonce, $Z_{3}$ : $\exp (m s g, m s g), U: \operatorname{kdf}(m s g)$ and all remaining pattern variables are of type msg.

$$
\begin{aligned}
f_{1}(\{|X, Y|\} Z) & =\left\langle f_{1}(X), f_{1}(Y)\right\rangle \\
f_{1}\left(\operatorname{mac}\left(X_{1}, \ldots, X_{8}\right)\right) & =\operatorname{mac}\left(\widehat{f}_{1}\left(\left[X_{1}, X_{3}, X_{5}, X_{6}, X_{7}, X_{8}\right]\right)\right) \\
f_{1}\left(\operatorname{mac}\left(Y_{1}, \ldots, Y_{8}\right)\right) & =\operatorname{mac}\left(\widehat{f_{1}}\left(\left[Y_{1}, Y_{5}, Y_{6}, Y_{7}, Y_{8}\right]\right)\right) \\
f_{1}\left(\operatorname{kdf}\left(Z_{1}, \ldots, Z_{5}\right)\right) & =\operatorname{kdf}\left(f_{1}\left(Z_{3}\right)\right) \\
f_{1}(\operatorname{prf}(U, Z)) & =f_{1}(U) \\
f_{1}\left(\exp \left(U_{1}, U_{2}\right)\right) & =\exp \left(f_{1}\left(U_{1}\right), f_{1}\left(U_{2}\right)\right), \\
f_{1}\left(\operatorname{sh}\left(U_{1}, U_{2}\right)\right) & =\operatorname{sh}\left(f_{1}\left(U_{1}\right), f_{1}\left(U_{2}\right)\right), \\
f_{1}\left(\left\langle U_{3}, U_{4}\right\rangle\right) & =\left\langle f_{1}\left(U_{3}\right), f_{1}\left(U_{4}\right)\right\rangle,
\end{aligned}
$$


There are only two equations in $A x_{c s}$, namely $\exp (\exp (g, X), Y) \simeq \exp (\exp (g, Y), X)$ and $\operatorname{sh}(X, Y) \simeq \operatorname{sh}(Y, X)$ where $X, Y: m s g$. Let $\tau \in \operatorname{subs}\left(\Pi\left(E_{f}\right)\right)$. For both axioms $\{s, t\} \in A x_{c s}$, it is not hard to see that $\tau \downarrow \cap \Gamma(s) \downarrow \neq \emptyset$ implies that $\tau=m s g$ or $\tau=\Gamma(s)=\Gamma(t)$. Hence, it holds that $\Gamma(s) \preccurlyeq \tau$ and $\Gamma(t) \preccurlyeq \tau$. Thus we conclude that $F_{1}$ is $R, A x$-closed.

\section{F.2 Syntactic criterion for injectiveness-like}

In this subsection, we present a syntactic criterion to justify the satisfaction of condition $\mathcal{I}$. More generally, we want to solve the following problem.

Problem 1. Suppose $F_{f}$ is $R, A x$-closed. Let $t, u \in \mathcal{M}^{\sharp}$ be terms and $\{t, u\} \subseteq$ $u \operatorname{dom}\left(F_{f}\right)$. Under which conditions it holds that $f(t \sigma)={ }_{E} f(u \sigma)$ implies that $t \sigma={ }_{E} u \sigma$ for all $R, A x$-normal well-typed ground substitutions $\sigma$ ?

We assume arbitrary but fixed terms $t$ and $u$ such that $t, u \in u d o m\left(F_{f}\right)$ and a $R, A x$-normal well-typed ground substitution $\sigma$. We intend to look for sufficient conditions under which $f(t \sigma)={ }_{E} f(u \sigma)$ implies $t \sigma={ }_{E} u \sigma$. We also require that these conditions do not depend on $\sigma$. This is to ensure that our conditions work for all such substitutions $\sigma$.

In the following paragraphs, we present criteria that can be applied when either no message variables are present in $t$ and $u$ or message variables occur only in $t$ or in $u$. The hardest case is when message variables are present in both $t$ and $u$, for which we currently do not have a good criterion.

Criterion I Note that by the substitution property, we can derive the following equalities:

$$
\begin{aligned}
f(t \sigma) & =f(t) f(\sigma), \\
f(u \sigma) & =f(u) f(\sigma) .
\end{aligned}
$$

Hence, the equality $t \sigma=_{E} u \sigma$ immediately follows from $f(t) f(\sigma)={ }_{E} f(u) f(\sigma)$ if it holds that $f(t)=t, f(u)=u$, and $X f(\sigma)=X \sigma$ for all $X \in \operatorname{vars}(t) \cup \operatorname{vars}(u)$. Clearly, we have that $f(X \sigma)=X \sigma$ for all non-message variables $X \in \operatorname{dom}(\sigma)$. Therefore, in the case that $t$ and $u$ do not contain message variables, the following syntactic criterion is straightforward.

Proposition 6. Let $t, u \in \mathcal{M}^{\sharp}$ be terms such that

(i) $m s g \notin \Gamma(\operatorname{vars}(t) \cup \operatorname{vars}(u))$,

(ii) $t, u \in u \operatorname{dom}\left(F_{f}\right)$.

If $f(t)=t$ and $f(u)=u$ then for all ground substitutions $\sigma$ that are well-typed, we have that $f(t \sigma)={ }_{E} f(u \sigma)$ implies $t \sigma={ }_{E} u \sigma$.

Proof. Without loss of generality, we can assume that $\operatorname{dom}(\sigma) \subseteq \operatorname{vars}(t) \cup$ $\operatorname{vars}(u)$. Since $m s g \notin \Gamma(\operatorname{vars}(t) \cup \operatorname{vars}(u))$, we derive that $X \sigma$ is an atom for 
all $X \in \operatorname{dom}(\sigma)$. This implies $f(\sigma)=\sigma$. Moreover, by Theorem 1, we have $f(t \sigma)=f(t) f(\sigma)$ and $f(u \sigma)=f(u) f(\sigma)$. From assumption, we derive

$$
\begin{gathered}
f(t \sigma)=t \sigma \\
f(u \sigma)=u \sigma
\end{gathered}
$$

Since $f(t \sigma)={ }_{E} f(u \sigma)$, we obtain that $t \sigma=_{E} u \sigma$ as required. This completes the proof of the proposition.

Note that for criterion I to be applicable, we require that $f$ is the identity for the terms in equalities. This is often the case, as these terms typically have simple structures and therefore it is reasonable to keep them unchanged.

Criterion II Here, we consider the case where $t$ or $u$ contain message variables. Without loss of generality, we assume that message variables occur only in $t$. Using the same approach as above, we assume that $f(t)=t, f(u)=u$. We want to be able to show that

$$
\left.f(\sigma)\right|_{\operatorname{vars}(t) \cup \operatorname{vars}(u)}=\left.\sigma\right|_{\operatorname{vars}(t) \cup \operatorname{vars}(u)} .
$$

Equivalently, we need to be able to show that $X f(\sigma)=X \sigma$ for every variable $X \in \operatorname{vars}(t) \cup \operatorname{vars}(u)$. This holds trivially for $X \in \operatorname{vars}(u)$ as shown above. To prove that $X f(\sigma)=X \sigma$ for each message variable $X \in \operatorname{vars}(t)$, we need to ensure that $f(\sigma)$ is $R, A x$-normal. Otherwise, equality (20) and injectivity property of Problem 1 may fail as illustrated in the following example.

Example 10. We consider the case that $t=X$ is a message variable, $u=a$ is an atom and $E_{f}$ contains the following equations:

$$
\begin{aligned}
f\left(\left\{\left|X_{1}\right|\right\}_{Y_{1}}\right) & =\left\{\left|f\left(X_{1}\right)\right|\right\}_{f\left(Y_{1}\right)} \\
f\left(\left\{\left|X_{2}\right|\right\}_{Y_{2}}^{-1}\right) & =\left\{\left|f\left(X_{2}\right)\right|\right\}_{f\left(Y_{2}\right)}^{-1} \\
f\left(\mathrm{~h}\left(X_{2}\right)\right) & =f\left(X_{2}\right)
\end{aligned}
$$

where all pattern variables are of type $m s g$. Intuitively, $f$ is homomorphic for $\{|\cdot|\}$. and $\{|\cdot|\}^{-1}$ and removes the hash function symbol h from terms. We define the substitution $\sigma=\left\{\left\{\{|a|\}_{\mathrm{h}(a)} \mid\right\}_{a}^{-1} / X\right\}$. Then we have $\operatorname{tf}(\sigma)={ }_{E} u f(\sigma)$ because $u f(\sigma)=a$ and

$$
t f(\sigma)=f(X \sigma)=f\left(\left\{\left|\{|a|\}_{\mathrm{h}(a)}\right|\right\}_{a}^{-1}\right)=\left\{\left|\{|a|\}_{a}\right|\right\}_{a}^{-1}={ }_{E} a .
$$

But $X \sigma=\left\{\{|a|\}_{\mathrm{h}(a)} \mid\right\}_{a}^{-1} \neq_{E} a=u \sigma$.

This example also highlights the difficulty of achieving $R, A x$-normality for $f(\sigma)$ without substantial restrictions. We therefore take the following approach. We show that for each $R, A x$-normal attack $\sigma$ on a property $\phi$ in $P$, there is an $R, A x$-normal attack $\sigma^{\prime}$ on $\phi$ such that $f\left(\sigma^{\prime}\right)$ is $R, A x$-normal. We construct $\sigma^{\prime}$ from $\sigma$ by replacing a subterm $v$ of the terms in $\operatorname{ran}(\sigma)$ such that $f(v)$ is not $R, A x$-normal with a new constant $a$. By applying this replacement exhaustively, 
we eliminate all subterms in the range of $\sigma$ whose corresponding abstracted terms are redexes. The resulting substitution $\sigma^{\prime}$ satisfies that $f\left(\sigma^{\prime}\right)$ is $R, A x$-normal.

We define the set of rewrite rules R3* such that $l \rightarrow r \in \mathrm{R}^{*}$ if and only if $l$ is of the form $c\left(u_{1}, \ldots, u_{n}\right)$ for some $c \in \Sigma^{n}$ and $u_{1}, \ldots, u_{n}$ are variables or constants and pairwise distinct. We overload notation and define the following sets:

$$
\begin{aligned}
\operatorname{subs}(A x) & =\bigcup_{\{s, t\} \in A x} \operatorname{subs}(s) \cup \operatorname{subs}(t), \\
c t(A x) & =\bigcup_{\{s, t\} \in A x} \operatorname{ct}(s) \cup \operatorname{ct}(t), \\
\operatorname{lh} s(R) & =\{l \mid l \rightarrow r \in R\}
\end{aligned}
$$

For terms $v$ and $v^{\prime}$, we use $\rho_{\left[v^{\prime} / v\right]}$ to denote the mapping that replaces each term $t^{\prime}$ such that $t^{\prime}={ }_{A x} v$ by $v^{\prime}$. We also define $\operatorname{dom}\left(\rho_{\left[v^{\prime} / v\right]}\right)=\{t \in \mathcal{T} \mid$ $\left.t={ }_{A x} v\right\}$. For a substitution $\sigma$, we denote by $\sigma \rho_{\left[v^{\prime} / v\right]}$ the substitution such that $\operatorname{dom}\left(\sigma \rho_{\left[v^{\prime} / v\right]}\right)=\operatorname{dom}(\sigma)$ and $\sigma \rho_{\left[v^{\prime} / v\right]}(X)=(X \sigma) \rho_{\left[v^{\prime} / v\right]}$. We call a term $t$ $R, A x$-stable if $t \sigma$ is $R, A x$-normal whenever $\sigma$ is $R, A x$-normal and well-typed. A set of terms is $R, A x$-stable if all its elements are. We also use $\mathcal{P}$ os $(t)$ to denote the set of all positions in a given term $t$.

Before establishing our criterion, we prove some auxiliary results.

Lemma 16. Let $u, t, v$ be ground terms and $a$ be an atom such that $a \notin$ subs $(u) \cup$ $\operatorname{subs}(t) \cup \operatorname{subs}(A x)$. Suppose that $u \rho_{[a / v]}={ }_{A x} t \rho_{[a / v]}$. Then $u \in \operatorname{dom}\left(\rho_{[a / v]}\right)$ if and only if $t \in \operatorname{dom}\left(\rho_{[a / v]}\right)$.

Proof. By symmetry, it is sufficient to show that $u \in \operatorname{dom}\left(\rho_{[a / v]}\right)$ implies $t \in$ $\operatorname{dom}\left(\rho_{[a / v]}\right)$. Suppose that $u \in \operatorname{dom}\left(\rho_{[a / v]}\right)$. Then we have $u \rho_{[a / v]}=a$. Together with $u \rho_{[a / v]}={ }_{A x} t \rho_{[a / v]}$ and $a$ is an atom, this implies $t \rho_{[a / v]}=a$. If $t \in \operatorname{dom}\left(\rho_{[a / v]}\right)$ then we are done. Otherwise, since $a \notin s u b s(t)$, there must be a strict subterm $t^{\prime}$ of $t$ such that $t^{\prime} \in \operatorname{dom}\left(\rho_{[a / v]}\right)$. This implies $a$ is a strict subterm of $t \rho_{[a / v]}$. From the fact that $a={ }_{A x} t \rho_{[a / v]}$ and $a \notin s u b s(A x)$, we obtain a contradiction and thus complete the proof of the lemma.

Lemma 17. Let $u, t, v$ be ground terms and $a$ be an atom such that $a \notin$ subs $(u) \cup$ $\operatorname{subs}(t) \cup \operatorname{subs}(A x)$. Then $u \rho_{[a / v]}=t \rho_{[a / v]}$ implies $u={ }_{A x} t$.

Proof. We show this lemma by induction on $u$.

- If $u$ is an atom we consider two cases.

- If $u \in \operatorname{dom}\left(\rho_{[a / v]}\right)$ then by Lemma 16, we have that $t \in \operatorname{dom}\left(\rho_{[a / v]}\right)$. This implies $u={ }_{A x} v$ and $t={ }_{A x} v$. Hence, we obtain $u={ }_{A x} t$.

- If $u \notin \operatorname{dom}\left(\rho_{[a / v]}\right)$ then by Lemma 16, we have that $t \notin \operatorname{dom}\left(\rho_{[a / v]}\right)$. Thus, we have $u \rho_{[a / v]}=u$ and $t \rho_{[a / v]}=t$. Therefore, we obtain $u=t$ and hence $u={ }_{A x} t$.

- If $u=g\left(u_{1}, \ldots, u_{n}\right)$ for some $g \in \Sigma^{n}$ then we consider two cases.

- If $u \in \operatorname{dom}\left(\rho_{[a / v]}\right)$ then by Lemma 16, we have $t \in \operatorname{dom}\left(\rho_{[a / v]}\right)$. It follows that $t \rho_{[a / v]}=a$. Together with the assumption that $a \notin s u b s(t)$, this yields $t \in \operatorname{dom}\left(\rho_{[a / v]}\right)$. Hence, we have $u={ }_{A x} t$ as required. 
- If $u \notin \operatorname{dom}\left(\rho_{[a / v]}\right)$ then by Lemma 16, we also have $t \notin \operatorname{dom}\left(\rho_{[a / v]}\right)$. Since $\operatorname{top}\left(t \rho_{[a / v]}\right)=g$ and $t \notin \operatorname{dom}\left(\rho_{[a / v]}\right)$, we must have $\operatorname{top}(t)=g$. Therefore, we have $t=g\left(t_{1}, \ldots, t_{n}\right)$. We derive that

$$
\begin{aligned}
u \rho_{[a / v]} & =g\left(u_{1} \rho_{[a / v]}, \ldots, u_{n} \rho_{[a / v]}\right), \\
t \rho_{[a / v]} & =g\left(t_{1} \rho_{[a / v]}, \ldots, t_{n} \rho_{[a / v]}\right) .
\end{aligned}
$$

Since $u \rho_{[a / v]}=t \rho_{[a / v]}$, we have $u_{i} \rho_{[a / v]}=t_{i} \rho_{[a / v]}$ for all $i \in \widetilde{n}$. By induction hypothesis, we know that $u_{i}={ }_{A x} t_{i}$. Therefore, by the Congruence rule, we conclude that $u={ }_{A x} t$ as required.

This completes the proof of the lemma.

Lemma 18. Let $t, u, v$ be terms and $a$ be an atom such that $t, v$ are ground and $a \notin \operatorname{subs}(t) \cup \operatorname{subs}(u) \cup \operatorname{subs}(A x)$. Let $\sigma$ be a ground substitution such that $\operatorname{dom}(\sigma)=\operatorname{vars}(u)$ and $t \rho_{[a / v]}=u \sigma$. Then there is a ground substitution $\sigma^{\prime}$ such that the following holds.

(i) $\operatorname{dom}\left(\sigma^{\prime}\right)=\operatorname{vars}(u)$,

(ii) $a \notin \operatorname{subs}\left(\operatorname{ran}\left(\sigma^{\prime}\right)\right)$,

(iii) $u \sigma^{\prime}={ }_{A x} t$, and

(iv) $\sigma^{\prime} \rho_{[a / v]}=\sigma$.

Proof. We prove this lemma by induction on $u$.

- If $u$ is an atom then we have $u \sigma=u$ and thus $t \rho_{[a / v]}=u$. Since $a \notin \operatorname{subs}(u)$, we must have $t \rho_{[a / v]}=t=u$. Thus we set $\sigma^{\prime}$ to the empty substitution and obtain $u \sigma^{\prime}=t$. Moreover, we also have $\operatorname{dom}\left(\sigma^{\prime}\right)=\emptyset=\operatorname{dom}(\sigma)$. Hence, it is clear that $\sigma^{\prime} \rho_{[a / v]}=\sigma$.

- If $u=X$ is a variable then let $\sigma^{\prime}$ be such that $\operatorname{dom}\left(\sigma^{\prime}\right)=\{X\}$ and $X \sigma^{\prime}=t$. Then we have $u \sigma^{\prime}=t$. By assumption, we have $a \notin \operatorname{subs}(X \sigma)=$ $\operatorname{subs}(\operatorname{ran}(\sigma))$. Moreover, since $t \rho_{[a / v]}=X \sigma$, it follows that $\left(X \sigma^{\prime}\right) \rho_{[a / v]}=$ $X \sigma$. Thus, we also have $\sigma^{\prime} \rho_{[a / v]}=\sigma$.

- If $u=g\left(u_{1}, \ldots, u_{n}\right)$ for some $g \in \Sigma^{n}$ then since $t \rho_{[a / v]}=u \sigma$ and $a$ is an atom, there must be terms $t_{1}, \ldots, t_{n}$ such that

$$
\begin{aligned}
t & =g\left(t_{1}, \ldots, t_{n}\right), \\
t \rho_{[a / v]} & =g\left(t_{1} \rho_{[a / v]}, \ldots, t_{n} \rho_{[a / v]}\right) .
\end{aligned}
$$

Therefore, we have $t_{i} \rho_{[a / v]}=u_{i} \sigma$ for all $i \in \widetilde{n}$. By induction hypothesis, there are ground substitutions $\sigma_{1}, \ldots, \sigma_{n}$ such that for all $i \in \widetilde{n}$, we have

$$
\begin{aligned}
& a \notin \operatorname{subs}\left(\operatorname{ran}\left(\sigma_{i}\right)\right), \\
& \operatorname{dom}\left(\sigma_{i}\right)=\operatorname{vars}\left(u_{i}\right), \\
& \sigma_{i} \rho_{[a / v]}=\left.\sigma\right|_{\text {vars }}\left(u_{i}\right), \text { and } \\
& u_{i} \sigma_{i}={ }_{A x} t_{i} .
\end{aligned}
$$

We define $\sigma^{\prime}$ such that $\operatorname{dom}\left(\sigma^{\prime}\right)=\operatorname{vars}(u)$ and for all $X \in \operatorname{dom}\left(\sigma^{\prime}\right), X \sigma^{\prime}=$ $X \sigma_{i}$ where $i \in \widetilde{n}$ is the smallest index such that $X \in \operatorname{dom}\left(\sigma_{i}\right)$. It is clear that 
$\sigma^{\prime} \rho_{[a / v]}=\sigma$ and $a \notin \operatorname{subs}\left(\operatorname{ran}\left(\sigma^{\prime}\right)\right)$. To see that $u \sigma^{\prime}={ }_{A x} t$, it is sufficient to show that for all $i, j \in \widetilde{n}$ and all $X \in \operatorname{dom}\left(\sigma_{i}\right) \cap \operatorname{dom}\left(\sigma_{j}\right)$, it holds that $X \sigma_{i}={ }_{A x} X \sigma_{j}$. Let $i, j \in \widetilde{n}$ and $X \in \operatorname{dom}\left(\sigma_{i}\right) \cap \operatorname{dom}\left(\sigma_{j}\right)$. From the induction hypothesis, we know that $\left(X \sigma_{i}\right) \rho_{[a / v]}=X \sigma$ and $\left(X \sigma_{j}\right) \rho_{[a / v]}=X \sigma$. Thus, we have $\left(X \sigma_{i}\right) \rho_{[a / v]}=\left(X \sigma_{j}\right) \rho_{[a / v]}$. This by Lemma 17 implies that $X \sigma_{i}=A x$ $X \sigma_{j}$. We therefore conclude this case.

Lemma 19. Let $t, u, v$ be ground terms and $a$ be an atom such that $t, v$ are ground and $a \notin \operatorname{subs}(t) \cup \operatorname{subs}(u) \cup \operatorname{subs}(A x)$. Suppose that

(i) $t \rho_{[a / v]}={ }_{A x} u$, and

(ii) $\operatorname{top}(v) \notin \operatorname{ct}(A x)$.

Then there is a ground term $t^{\prime}$ such that $t={ }_{A x} t^{\prime}$ and $t^{\prime} \rho_{[a / v]}=u$.

Proof. We prove this lemma by induction on the derivation of $t \rho_{[a / v]}={ }_{A x} u$ depending on the last rule that has been applied.

- Reflexivity: We have $t \rho_{[a / v]}=u$. Thus, we can pick $t^{\prime}=t$.

- Axiom: In this case, there are $\left\{s, s^{\prime}\right\} \in A x$ and a ground substitution $\sigma$ such that $t \rho_{[a / v]}=s \sigma$ and $u=s^{\prime} \sigma$. By Lemma 18, there exists a ground substitutions $\sigma^{\prime}$ such that

$$
\begin{aligned}
& \operatorname{dom}\left(\sigma^{\prime}\right)=\operatorname{vars}(s), \\
& a \notin \operatorname{subs}\left(\operatorname{ran}\left(\sigma^{\prime}\right)\right), \\
& s \sigma^{\prime}={ }_{A x} t, \text { and } \\
& \sigma^{\prime} \rho_{[a / v]}=\sigma .
\end{aligned}
$$

We pick $t^{\prime}=s^{\prime} \sigma^{\prime}$. Since vars $(s)=\operatorname{vars}\left(s^{\prime}\right)$, we have $s^{\prime} \sigma^{\prime}$ is ground. Moreover, we have $t^{\prime}=s_{A x} t$. By assumption (ii), we derive that $s^{\prime} \sigma=s^{\prime}\left(\sigma^{\prime} \rho_{[a / v]}\right)=$ $\left(s^{\prime} \sigma^{\prime}\right) \rho_{[a / v]}=t^{\prime} \rho_{[a / v]}$. Hence, we have $u=t^{\prime} \rho_{[a / v]}$ as required.

- Congruence: In this case, there are $c \in \Sigma^{n}$ and terms $t_{1}, \ldots, t_{n}, u_{1}, \ldots, u_{n}$ such that

$$
\begin{aligned}
& t=c\left(t_{1}, \ldots, t_{n}\right), \\
& u=c\left(u_{1}, \ldots, u_{n}\right), \\
& t_{i} \rho_{[a / v]}={ }_{A x} u_{i} \text { for all } i \in \widetilde{n} .
\end{aligned}
$$

By induction hypothesis, there is a term $t_{i}^{\prime}$ such that $t_{i}={ }_{A x} t_{i}^{\prime}$ and $u_{i}=$ $t_{i}^{\prime} \rho_{[a / v]}$ for all $i \in \tilde{n}$. We define $t^{\prime}=c\left(t_{1}^{\prime}, \ldots, t_{n}^{\prime}\right)$ and derive that $t^{\prime}=_{A x} t$ and $u=t^{\prime} \rho_{[a / v]}$ as required.

- Transitivity: In this case, there is a term $w$ such that $t \rho_{[a / v]}={ }_{A x} w$ and $w={ }_{A x} u$. By induction hypothesis, there is a term $w^{\prime}$ such that $t={ }_{A x} w^{\prime}$ and $w=w^{\prime} \rho_{[a / v]}$. Thus, we have $w^{\prime} \rho_{[a / v]}={ }_{A x} u$. Using the induction hypothesis, we derive that there exists a term $t^{\prime}$ such that $w^{\prime}={ }_{A x} t^{\prime}$ and $u=t^{\prime} \rho_{[a / v]}$. It follows that $t={ }_{A x} t^{\prime}$ which concludes this case.

Lemma 20. Let $u, t, v$ be ground terms and a be an atom. Suppose that for all pairs $\left\{s, s^{\prime}\right\} \in A x$, we have top $(v) \notin \operatorname{ct}(A x)$. Then $u={ }_{A x} t$ implies $u \rho_{[a / v]}={ }_{A x}$ $t \rho_{[a / v]}$. 
Proof. We prove the direction from left to right by induction on the derivation $u={ }_{A x} t$ depending on the last rule that has been applied.

- Reflexivity: In this case, we have $u=t$. Thus it is obvious that $u \rho_{[a / v]}=$ $t \rho_{[a / v]}$.

- Axiom: In this case, then there are a pair $\left\{s, s^{\prime}\right\} \in A x$ and a substitution $\sigma$ such that $u=s \sigma$ and $t=s^{\prime} \sigma$. Let $\sigma^{\prime}=\sigma \rho_{[a / v]}$. Since $\operatorname{top}(v) \notin c t(A x)$, we have

$$
\begin{aligned}
(s \sigma) \rho_{[a / v]} & =s \sigma^{\prime}, \\
\left(s^{\prime} \sigma\right) \rho_{[a / v]} & =s^{\prime} \sigma^{\prime} .
\end{aligned}
$$

Therefore, we derive

$$
\begin{aligned}
& u \rho_{[a / v]}=(s \sigma) \rho_{[a / v]}=s \sigma^{\prime}, \\
& t \rho_{[a / v]}=\left(s^{\prime} \sigma\right) \rho_{[a / v]}=s^{\prime} \sigma^{\prime} .
\end{aligned}
$$

Using the Axiom rule, we derive that $u \rho_{[a / v]}={ }_{A x} t \rho_{[a / v]}$ as required.

- Congruence: In this case, we have that $u=g\left(u_{1}, \ldots, u_{n}\right)$ and $t=g\left(t_{1}, \ldots, t_{n}\right)$ for some $g \in \Sigma^{n}, n \geq 1$. Moreover, we have $u_{i}={ }_{A x} t_{i}$ for all $i \in \widetilde{n}$. Since $u={ }_{A x} t$, it is clear that $u \in \operatorname{dom}\left(\rho_{[a / v]}\right)$ if and only if $t \in \operatorname{dom}\left(\rho_{[a / v]}\right)$. We consider two cases.

- If $u \in \operatorname{dom}\left(\rho_{[a / v]}\right)$ then $t \in \operatorname{dom}\left(\rho_{[a / v]}\right)$. Thus, we have $u \rho_{[a / v]}=a=$ $t \rho_{[a / v]}$.

- If $u \notin \operatorname{dom}\left(\rho_{[a / v]}\right)$ then $t \notin \operatorname{dom}\left(\rho_{[a / v]}\right)$. Therefore, we have

$$
\begin{aligned}
u \rho_{[a / v]} & =g\left(u_{1} \rho_{[a / v]}, \ldots, u_{n} \rho_{[a / v]}\right), \\
t \rho_{[a / v]} & =g\left(t_{1} \rho_{[a / v]}, \ldots, t_{n} \rho_{[a / v]}\right) .
\end{aligned}
$$

Moreover, by induction hypothesis, we know that $u_{i} \rho_{[a / v]}={ }_{A x} t_{i} \rho_{[a / v]}$ for all $i \in \widetilde{n}$. Hence, we obtain $u \rho_{[a / v]}={ }_{A x} t \rho_{[a / v]}$ as required.

- Transitivity: In this case, there is a term $w$ such that $u={ }_{A x} w$ and $w={ }_{A x} t$. By induction hypothesis, we have

$$
\begin{aligned}
u \rho_{[a / v]} & ={ }_{A x} w \rho_{[a / v]}, \\
w \rho_{[a / v]} & ={ }_{A x} t \rho_{[a / v]} .
\end{aligned}
$$

It follows that $u \rho_{[a / v]}={ }_{A x} t \rho_{[a / v]}$ as required.

This completes the proof of the lemma.

Lemma 21. Let $u, t, v$ be ground terms and $a$ be an atom such that

(i) $a \notin \operatorname{subs}(u) \cup \operatorname{subs}(t) \cup \operatorname{subs}(A x)$,

(ii) $\operatorname{top}(v) \notin c t(A x)$, and

(iii) for all $\left\{s, s^{\prime}\right\} \in A x$, we have vars $(s)=\operatorname{vars}\left(s^{\prime}\right)$.

Then $u \rho_{[a / v]}={ }_{A x} t \rho_{[a / v]}$ implies $u={ }_{A x} t$.

Proof. We prove this lemma by induction on the derivation $u \rho_{[a / v]}={ }_{A x} t \rho_{[a / v]}$. 
- Reflexivity: In this case, we have $u \rho_{[a / v]}=t \rho_{[a / v]}$. By Lemma 17, we have $u={ }_{A x} t$ as required.

- Axiom: In this case, there are a pair $\left\{s, s^{\prime}\right\} \in A x$ and a ground substitution $\sigma$ such that $\operatorname{dom}(\sigma)=\operatorname{vars}(s) \cup \operatorname{vars}\left(s^{\prime}\right)$ and

$$
\begin{aligned}
& u \rho_{[a / v]}=s \sigma, \\
& t \rho_{[a / v]}=s^{\prime} \sigma .
\end{aligned}
$$

Moreover, by Lemma 18, there is a ground substitution $\sigma^{\prime}$ such that $d o m\left(\sigma^{\prime}\right)=$ $\operatorname{vars}(s)$ and

$$
\begin{aligned}
u & ={ }_{A x} s \sigma^{\prime} \\
\sigma^{\prime} \rho_{[a / v]} & =\left.\sigma\right|_{\text {vars }(s)} .
\end{aligned}
$$

Since $\operatorname{vars}(s)=\operatorname{vars}\left(s^{\prime}\right)$, we derive that $\left.\sigma\right|_{\text {vars }(s)}=\sigma$. Hence, we have

$$
s^{\prime} \sigma=s^{\prime}\left(\sigma^{\prime} \rho_{[a / v]}\right)
$$

By assumption, for all terms $w \in \operatorname{dom}\left(\rho_{[a / v]}\right) \cap \operatorname{subs}\left(s^{\prime} \sigma\right)$, we have $w \in$ $\operatorname{subs}(\operatorname{ran}(\sigma))$. Therefore, we must have $s^{\prime} \sigma=\left(s^{\prime} \sigma^{\prime}\right) \rho_{[a / v]}$. Hence, we obtain

$$
t \rho_{[a / v]}=\left(s^{\prime} \sigma^{\prime}\right) \rho_{[a / v]} .
$$

By Lemma 17, we derive that $t={ }_{A x} s^{\prime} \sigma^{\prime}$. Together with $u={ }_{A x} s \sigma^{\prime}$, we have $u={ }_{A x} t$ as required.

- Congruence: In this case, there is $g \in \Sigma^{n}$ such that

$$
\begin{aligned}
u \rho_{[a / v]} & =g\left(u_{1}, \ldots, u_{n}\right), \\
t \rho_{[a / v]} & =g\left(t_{1}, \ldots, t_{n}\right) .
\end{aligned}
$$

Moreover, we have $u_{i}={ }_{A x} t_{i}$ for all $i \in \widetilde{n}$. We consider two cases.

- If $u \in \operatorname{dom}\left(\rho_{[a / v]}\right)$ then by Lemma 16, we have $t \in \operatorname{dom}\left(\rho_{[a / v]}\right)$. Therefore, we have $u={ }_{A x} t$ as required.

- If $u \notin \operatorname{dom}\left(\rho_{[a / v]}\right)$ then by Lemma 16, we also have $t \notin d o m\left(\rho_{[a / v]}\right)$. Thus, there must be terms $u_{1}^{\prime}, \ldots, u_{n}^{\prime}$ and terms $t_{1}^{\prime}, \ldots, t_{n}^{\prime}$ such that

$$
\begin{aligned}
u & =g\left(u_{1}^{\prime}, \ldots, u_{n}^{\prime}\right), \\
t & =g\left(t_{1}^{\prime}, \ldots, t_{n}^{\prime}\right), \\
u_{i}^{\prime} \rho_{[a / v]} & =u_{i} \text { for all } i \in \widetilde{n}, \\
t_{i}^{\prime} \rho_{[a / v]} & =t_{i} \text { for all } i \in \widetilde{n} .
\end{aligned}
$$

Hence, we have $u_{i}^{\prime} \rho_{[a / v]}={ }_{A x} t_{i}^{\prime} \rho_{[a / v]}$ for all $i \in \widetilde{n}$. By induction hypothesis, we know that $u_{i}^{\prime}={ }_{A x} t_{i}^{\prime}$ for all $i \in \widetilde{n}$. This implies $u={ }_{A x} t$ as required.

- Transitivity: In this case, there is a term $w$ such that $u \rho_{[a / v]}={ }_{A x} w$ and $w={ }_{A x} t \rho_{[a / v]}$. We consider two cases.

- If $a \in \operatorname{subs}\left(u \rho_{[a / v]}\right) \cup \operatorname{subs}\left(t \rho_{[a / v]}\right)$ then since $a \notin \operatorname{subs}(A x)$, we derive that $a \in \operatorname{subs}(w)$. Hence, there is a term $w^{\prime}$ such that $w=w^{\prime} \rho_{[a / v]}$. By induction hypothesis, we have $u={ }_{A x} w^{\prime}$ and $w^{\prime}={ }_{A x} t$. This implies $u={ }_{A x} t$ as required. 
- If $a \notin \operatorname{subs}\left(u \rho_{[a / v]}\right) \cup \operatorname{subs}\left(t \rho_{[a / v]}\right)$ then we have $u \rho_{[a / v]}=u$ and $t \rho_{[a / v]}=$ $t$. Thus, we obtain $u={ }_{A x} t$ as required.

This completes the proof of the lemma.

Lemma 22. Let $t, u, v$ be terms and $a$ be an atom such that $t, v$ are ground such that

(i) $a \notin \operatorname{subs}(t) \cup \operatorname{subs}(u) \cup \operatorname{subs}(A x)$,

(ii) $\operatorname{top}(v) \notin c t(A x)$, and

(iii) for all $\left\{s, s^{\prime}\right\} \in A x$, we have that vars $(s)=\operatorname{vars}\left(s^{\prime}\right)$.

Let $\sigma$ be a ground substitution such that $\operatorname{dom}(\sigma)=\operatorname{vars}(u)$ and $t \rho_{[a / v]}={ }_{A x} u \sigma$. Then there is a ground substitution $\sigma^{\prime}$ such that the following holds.

$-\operatorname{dom}\left(\sigma^{\prime}\right)=\operatorname{vars}(u)$,

- $a \notin \operatorname{subs}\left(\operatorname{ran}\left(\sigma^{\prime}\right)\right)$,

$-u \sigma^{\prime}{ }_{A x} t$, and

$-\sigma^{\prime} \rho_{[a / v]}=\sigma$.

Proof. We prove this lemma by induction on the derivation $t \rho_{[a / v]}={ }_{A x} u \sigma$.

- Reflexivity: We have $t \rho_{[a / v]}=u \sigma$ and thus the conclusion follows immediately from Lemma 18.

- Axiom: Suppose that there are a pair $\left\{s, s^{\prime}\right\} \in A x$ and a ground substitution $\theta$ such that $\operatorname{dom}(\theta)=\operatorname{dom}(s) \cup \operatorname{dom}\left(s^{\prime}\right)$ and

$$
\begin{aligned}
t \rho_{[a / v]} & =s \theta, \\
u \sigma & =s^{\prime} \theta .
\end{aligned}
$$

By Lemma 18, there is a ground substitution $\sigma^{\prime \prime}$ such that

$$
\begin{aligned}
& \operatorname{dom}\left(\sigma^{\prime \prime}\right)=\operatorname{vars}(s), \\
& a \notin \operatorname{subs}\left(\operatorname{ran}\left(\sigma^{\prime \prime}\right)\right), \\
& \sigma^{\prime \prime} \rho_{[a / v]}=\left.\theta\right|_{\text {vars }(s)}, \text { and } \\
& t={ }_{A x} s \sigma^{\prime \prime} .
\end{aligned}
$$

Since $\operatorname{vars}(s)=\operatorname{vars}\left(s^{\prime}\right)$, we have $\left.\theta\right|_{\operatorname{vars}(s)}=\theta$. Hence, we derive that $s^{\prime} \theta=$ $s^{\prime}\left(\sigma^{\prime \prime} \rho_{[a / v]}\right)$. This by assumption implies that $s^{\prime} \theta=\left(s^{\prime} \sigma^{\prime \prime}\right) \rho_{[a / v]}$. Since $a \notin$ $\operatorname{subs}\left(\operatorname{ran}\left(\sigma^{\prime \prime}\right)\right)$, from assumption (ii), we have $a \notin \operatorname{subs}\left(s^{\prime} \sigma^{\prime \prime}\right)$. Moreover, we have $u \sigma=s^{\prime} \theta=\left(s^{\prime} \sigma^{\prime \prime}\right) \rho_{[a / v]}$. By Lemma 18, there is a ground substitution $\sigma^{\prime}$ such that

$$
\begin{aligned}
& \operatorname{dom}\left(\sigma^{\prime}\right)=\operatorname{vars}(u), \\
& a \notin \operatorname{subs}\left(\operatorname{ran}\left(\sigma^{\prime}\right)\right), \\
& \sigma^{\prime} \rho_{[a / v]}=\sigma, \text { and } \\
& s^{\prime} \sigma^{\prime \prime}={ }_{A x} u \sigma^{\prime} .
\end{aligned}
$$

Together with $t={ }_{A x} s \sigma^{\prime \prime}$, we derive that $u \sigma^{\prime}={ }_{A x} t$. 
- Congruence: We have $t=c\left(t_{1}, \ldots, t_{n}\right)$ and $u=c\left(u_{1}, \ldots, u_{n}\right)$ for $c \in \Sigma^{n}$. Moreover, we have $t_{i} \rho_{[a / v]}={ }_{A x} u_{i} \sigma$ for all $i \in \widetilde{n}$. By induction hypothesis, there are ground substitutions $\sigma_{i}$ for all $i \in \widetilde{n}$ such that

$$
\begin{aligned}
& \operatorname{dom}\left(\sigma_{i}\right)=\operatorname{vars}\left(u_{i}\right), \\
& a \notin \operatorname{subs}\left(\operatorname{ran}\left(\sigma_{i}\right)\right), \\
& u_{i} \sigma_{i}={ }_{A x} t_{i}, \text { and } \\
& \sigma_{i} \rho_{[a / v]}=\sigma .
\end{aligned}
$$

We can pick $\sigma^{\prime}=\bigcup_{i=1}^{n} \sigma_{i}$ that satisfies the desired properties.

- Transitivity: In this case, there is a term $w$ such that $t \rho_{[a / v]}={ }_{A x} w$ and $w={ }_{A x} u \sigma$. By Lemma 19, there is a ground term $t^{\prime}$ such that $t=_{A x} t^{\prime}$ and $w=t^{\prime} \rho_{[a / v]}$. Hence, we have $t^{\prime} \rho_{[a / v]}={ }_{A x} u \sigma$. By induction hypothesis, there exists a ground substitution $\sigma^{\prime}$ such that

$$
\begin{aligned}
& \operatorname{dom}\left(\sigma^{\prime}\right)=\operatorname{vars}(u), \\
& a \notin \operatorname{subs}\left(\operatorname{ran}\left(\sigma^{\prime}\right)\right), \\
& u \sigma_{i}=A x t^{\prime}, \text { and } \\
& \sigma^{\prime} \rho_{[a / v]}=\sigma .
\end{aligned}
$$

Since $t^{\prime}={ }_{A x} t$, we derive that $\sigma^{\prime}$ satisfies the desired properties.

This completes the proof of the lemma.

Lemma 23. Let $t, v$ be ground terms and $a$ be an atom such that $a \notin s u b s(t)$. Suppose that

(i) $t$ is $R$,Ax-normal,

(ii) $a \notin \operatorname{subs}(A x) \cup \operatorname{subs}(\operatorname{lhs}(R))$,

(iii) $\operatorname{top}(v) \notin \operatorname{ct}(A x)$, and

(iv) for all $\left\{s, s^{\prime}\right\} \in A x$, we have that vars $(s)=\operatorname{vars}\left(s^{\prime}\right)$.

Then $t \rho_{[a / v]}$ is $R, A x$-normal.

Proof. We prove this lemma by induction on $t$.

- If $t$ is an atom then we have $t \rho_{[a / v]}=t$ and thus $t \rho_{[a / v]}$ is $R, A x$-normal.

- If $t=g\left(t_{1}, \ldots, t_{n}\right)$ for some $g \in \Sigma^{n}$ then we consider two cases.

- If $t \in \operatorname{dom}\left(\rho_{[a / v]}\right)$ then we have $t \rho_{[a / v]}=a$ and thus $t \rho_{[a / v]}$ is $R, A x$-normal.

- If $t \notin \operatorname{dom}\left(\rho_{[a / v]}\right)$ then we have

$$
t \rho_{[a / v]}=g\left(t_{1} \rho_{[a / v]}, \ldots, t_{n} \rho_{[a / v]}\right)
$$

Since $t$ is $R, A x$-normal, so is $t_{i}$ for all $i \in \widetilde{n}$. By induction hypothesis, we have $t_{i} \rho_{[a / v]}$ is $R, A x$-normal for all $i \in \widetilde{n}$. There are two smaller cases.

* If there is no rule $l \rightarrow r \in R$ that is applicable to $t \rho_{[a / v]}$ at the root, then since $t_{i} \rho_{[a / v]}$ is $R, A x$-normal for all $i \in \widetilde{n}$, we derive that $t \rho_{[a / v]}$ is $R, A x$-normal. 
* If there is a rule $l \rightarrow r \in R$ that is applicable to $t \rho_{[a / v]}$ at the root, then there is a ground substitution $\sigma$ such that $\operatorname{dom}(\sigma)=\operatorname{vars}(l)$ and $t \rho_{[a / v]}={ }_{A x} l \sigma$. By Lemma 22, there is a ground substitution $\sigma^{\prime}$ such that $t={ }_{A x} l \sigma^{\prime}$. Thus means $t$ is not $R, A x$-normal which is a contradiction.

This completes the proof of the lemma.

Lemma 24. Let $t, v$ be ground terms such that $v$ is $R, A x$-normal and $a$ be an atom. Suppose that

(i) $a \notin \operatorname{subs}(t) \cup \operatorname{subs}(A x) \cup \operatorname{subs}($ lhs $(R))$,

(ii) $\operatorname{top}(v) \notin c t(A x)$,

(iii) top $(v) \notin c t(l) \backslash\{$ top $(l)\}$ for all $l \in l h s(R)$, and

(iv) for all $\left\{s, s^{\prime}\right\} \in A x$, we have that vars $(s)=\operatorname{vars}\left(s^{\prime}\right)$.

Then $\left(t \rho_{[a / v]}\right) \downarrow_{R, A x}={ }_{A x} t \downarrow_{R, A x} \rho_{[a / v]}$.

Proof. We prove this lemma by induction on the length $\ell$ of the derivation $t \rightarrow_{R, A x} t_{1} \rightarrow_{R, A x} \cdots \rightarrow_{R, A x} t_{\ell-1} \rightarrow_{R, A x} t \downarrow_{R, A x}$.

- If $\ell=0$ then $t$ is $R, A x$-normal. By Lemma 23, $t \rho_{[a / v]}$ is $R, A x$-normal. Therefore, we have $t \rho_{[a / v]}=A x\left(t \rho_{[a / v]}\right) \downarrow_{R, A x}$. Moreover, by Lemma 20, we have $t \rho_{[a / v]}={ }_{A x} t \downarrow_{R, A x} \rho_{[a / v]}$. Hence $\left(t \rho_{[a / v]}\right) \downarrow_{R, A x}={ }_{A x} t \downarrow_{R, A x} \rho_{[a / v]}$.

- If $\ell>0$ then there are a position $k$, a rule $l \rightarrow r \in R$, and a substitution $\sigma$ such that $\left.t\right|_{k}={ }_{A x} l \sigma$ and $\left(t[r \sigma]_{k}\right) \downarrow_{R, A x}={ }_{A x} t \downarrow_{R, A x}$. By Lemma 20, we have $\left.t\right|_{k} \rho_{[a / v]}={ }_{A x}(l \sigma) \rho_{[a / v]}$. Let $\sigma^{\prime}=\sigma \rho_{[a / v]}$.

Note that for all terms $u \in \operatorname{subs}(t)$ such that $\left.t\right|_{k} \in \operatorname{subs}(u)$, we must have

$$
u \notin \operatorname{dom}\left(\rho_{[a / v]}\right) .
$$

Suppose that it is not the case, i.e., we have $u={ }_{A x} v$. Then since $t_{k} \in$ $\operatorname{subs}(u)$ and $\left.t\right|_{k} \rightarrow_{R, A x} r \sigma$, we derive that $v$ is not $R, A x$-normal which is a contradiction. Therefore, we have established (21). In particular, we have $l \sigma \notin \operatorname{dom}\left(\rho_{[a / v]}\right)$. Moreover, there must not exist a non-variable term $u \in$ $\operatorname{subs}(l) \backslash\{l\}$ such that $u \sigma={ }_{A x} v$. Otherwise, by point (iii) in Definition 1, $u \sigma={ }_{A x} v$ implies $\operatorname{top}(u \sigma)=\operatorname{top}(v)$. This means $\operatorname{top}(u)=\operatorname{top}(v)$ which contradicts assumption (iv). Therefore, we have $(l \sigma) \rho_{[a / v]}=l\left(\sigma \rho_{[a / v]}\right)=l \sigma^{\prime}$. It follows that $\left.t\right|_{k} \rho_{[a / v]}={ }_{A x} l \sigma^{\prime}$. Hence, we obtain $t\left[\left.t\right|_{k} \rho_{[a / v]}\right]_{k}={ }_{A x} t\left[l \sigma^{\prime}\right]_{k}$. This yields the following.

$$
\begin{aligned}
t \rho_{[a / v]} & =\left(t\left[\left.t\right|_{k} \rho_{[a / v]}\right]_{k}\right) \rho_{[a / v]} \\
& ={ }_{A x}\left(t\left[l \sigma^{\prime}\right]_{k}\right) \rho_{[a / v]} \text { by Lemma } 20 .
\end{aligned}
$$

Together with (21), we derive that $\left(t\left[l \sigma^{\prime}\right]_{k}\right) \rho_{[a / v]} \rightarrow_{R, A x}\left(t\left[r \sigma^{\prime}\right]_{k}\right) \rho_{[a / v]}$. Thus, we have $\left(t_{[a / v]}\right) \downarrow_{R, A x}=A_{A x}\left(\left(t\left[r \sigma^{\prime}\right]_{k}\right) \rho_{[a / v]}\right) \downarrow_{R, A x}$. Note that from assumption (iii) and the fact that $r \in \operatorname{subs}(l)$, we derive that $r \sigma^{\prime}=r\left(\sigma \rho_{[a / v]}\right)=$ $(r \sigma) \rho_{[a / v]}$. Therefore, we have

$$
\begin{aligned}
\left(t\left[r \sigma^{\prime}\right]_{k}\right) \rho_{[a / v]} & =\left(t\left[(r \sigma) \rho_{[a / v]}\right]_{k}\right) \rho_{[a / v]} \\
& =\left(t[r \sigma]_{k}\right) \rho_{[a / v]} .
\end{aligned}
$$


Hence we have

$$
\left(t \rho_{[a / v]}\right) \downarrow_{R, A x}={ }_{A x}\left(\left(t[r \sigma]_{k}\right) \rho_{[a / v]}\right) \downarrow_{R, A x}
$$

Since $\left(t[r \sigma]_{k}\right) \downarrow_{R, A x}={ }_{A x} t \downarrow_{R, A x}$, by Lemma 20, we have

$$
\left(t[r \sigma]_{k}\right) \downarrow_{R, A x} \rho_{[a / v]}={ }_{A x} t \downarrow_{R, A x} \rho_{[a / v]} \cdot
$$

By induction hypothesis, we have

$$
\left(\left(t[r \sigma]_{k}\right) \rho_{[a / v]}\right) \downarrow_{R, A x}={ }_{A x}\left(t[r \sigma]_{k}\right) \downarrow_{R, A x} \rho_{[a / v]} .
$$

This by (22) and (23) yields $\left(t \rho_{[a / v]}\right) \downarrow_{R, A x}={ }_{A x} t \downarrow_{R, A x} \rho_{[a / v]}$ as required.

This completes the proof of the lemma.

Lemma 25. Let $\{t, u, a, v\}$ be ground terms such that top $(v) \notin \operatorname{ct}(A x)$ and $a$ is an atom. Then $t={ }_{E} u$ if and only if that $t \rho_{[a / v]}={ }_{E} u \rho_{[a / v]}$.

Proof. We present all the derivation steps as follows.

$$
\begin{aligned}
t & ={ }_{E} \quad u \\
\Leftrightarrow t \downarrow_{R, A x} & ={ }_{A x} u \downarrow_{R, A x} \\
\Leftrightarrow t \downarrow_{R, A x} \rho_{[a / v]} & ={ }_{A x} u \downarrow_{R, A x} \rho_{[a / v]} \text { by Lemmas } 20,21 \\
\Leftrightarrow t \downarrow_{R, A x} \rho_{[a / v]} & ={ }_{A x} u \downarrow_{R, A x} \rho_{[a / v]} \text { by Lemma } 24 \\
\Leftrightarrow t \rho_{[a / v]} & ={ }_{E} \quad u \rho_{[a / v]} .
\end{aligned}
$$

This completes the proof of the lemma.

Lemma 26. Let $T \cup\{t, v\}$ be a set of ground terms such that $v$ is $R, A x$-normal and $a$ be a constant. Suppose that

(i) $a \notin \operatorname{subs}(t) \cup \operatorname{subs}(A x) \cup \operatorname{subs}(\operatorname{lhs}(R))$,

(ii) $\operatorname{top}(v) \notin \operatorname{ct}(A x)$,

(iii) $\operatorname{top}(v) \notin \operatorname{ct}(l) \backslash\{\operatorname{top}(l)\}$ for all $l \in \operatorname{lhs}(R)$, and

(iv) for all $\left\{s, s^{\prime}\right\} \in A x$, we have that $\operatorname{vars}(s)=\operatorname{vars}\left(s^{\prime}\right)$.

Then $T \vdash_{E} t$ implies $T \rho_{[a / v]}, I K_{0} \vdash_{E} t \rho_{[a / v]}$.

Proof. We prove this lemma induction on the derivation of $T \vdash_{E} t$ depending on the last rule that has been applied.

- Ax: We have $t \in T$ and thus $t \rho_{[a / v]} \in T \rho_{[a / v]}$. Therefore $T \rho_{[a / v]}, I K_{0} \vdash_{E}$ $t \rho_{[a / v]}$.

- Comp: We have $t=g\left(u_{1}, \ldots, t_{n}\right)$ and $T \vdash_{E} u_{i}$ for $i \in \widetilde{n}$. There are two cases.

- If $t={ }_{A x} v$ then we have $t \rho_{[a / v]}=a$. Since $a \in I K_{0}$, we obtain that

$$
T \rho_{[a / v]}, I K_{0} \vdash_{E} t \rho_{[a / v]} .
$$


- If $t \neq_{A x} v$ then we have

$$
t \rho_{[a / v]}=g\left(u_{1} \rho_{[a / v]}, \ldots, u_{n} \rho_{[a / v]}\right) .
$$

Moreover, by induction hypothesis, we have $T \rho_{[a / v]}, I K_{0} \vdash_{E} u_{i} \rho_{[a / v]}$ for all $i \in \widetilde{n}$. Hence, we obtain $T \rho_{[a / v]}, I K_{0} \vdash_{E} t \rho_{[a / v]}$ as required.

- Eq: In this case, there is a term $t^{\prime}$ such that $T \vdash_{E} t^{\prime}$ and $t^{\prime}={ }_{E} t$. Since $a \notin \operatorname{subs}(t)$, from assumption (i), we derive that $a \notin s u b s\left(t^{\prime}\right)$. Hence, we can apply the induction hypothesis and obtain $T \rho_{[a / v]} \vdash_{E} t^{\prime} \rho_{[a / v]}$. By Lemma 25, we have that $t^{\prime} \rho_{[a / v]}={ }_{E} t \rho_{[a / v]}$. Thus, we derive $T \rho_{[a / v]} \vdash_{E} t \rho_{[a / v]}$ as desired.

This completes the proof of the lemma.

Lemma 27. Let $T \cup\{t, v, a\}$ be terms such that $v$ is ground, a is a constant, and $a \notin \operatorname{subs}(t)$. Let $\sigma$ be a ground $R, A x$-normal well-typed substitution. Suppose that the following holds.

(i) $T \cup\{t\} \subseteq \operatorname{udom}\left(F_{f}\right)$,

(ii) for all terms $u \in \operatorname{subs}(T) \cup \operatorname{subs}(t)$, we have $f(u)$ is $R$, Ax-stable,

(iii) for all non-variable $t^{\prime} \in \operatorname{subs}(T) \cup \operatorname{subs}(t)$, we have $t^{\prime} \sigma \neq_{A x} v$,

(iv) $a \notin \operatorname{subs}(A x) \cup \operatorname{subs}(\operatorname{lhs}(R))$,

(v) $\operatorname{top}(v) \notin \operatorname{ct}(A x)$,

(vi) top $(v) \notin \operatorname{ct}(l) \backslash\{\operatorname{top}(l)\}$ for all $l \in \operatorname{lhs}(R)$, and

(vii) for all $\left\{s, s^{\prime}\right\} \in A x$, we have that $\operatorname{vars}(s)=\operatorname{vars}\left(s^{\prime}\right)$.

Then $T \sigma, I K_{0} \vdash_{E} t \sigma$ implies $T\left(\sigma \rho_{[a / v]}\right), I K_{0} \vdash_{E} t\left(\sigma \rho_{[a / v]}\right)$.

Proof. Suppose that $T \sigma, I K_{0} \vdash_{E} t \sigma$. By Lemma 26, we have

$$
(T \sigma) \rho_{[a / v]}, I K_{0} \rho_{[a / v]}, I K_{0} \vdash_{E}(t \sigma) \rho_{[a / v]} .
$$

By assumption (iii), this implies

$$
\begin{aligned}
(T \sigma) \rho_{[a / v]} & =T\left(\sigma \rho_{[a / v]}\right) \\
(t \sigma) \rho_{[a / v]} & =t\left(\sigma \rho_{[a / v]}\right) .
\end{aligned}
$$

Therefore, we obtain

$$
T\left(\sigma \rho_{[a / v]}\right), I K_{0} \vdash_{E} t\left(\sigma \rho_{[a / v]}\right) .
$$

This completes the proof of the lemma.

Lemma 28. Let $t, v$ be ground term, and a be an atom. Assume that

(i) $t$ is $R$, Ax-normal,

(ii) $v$ is composed and not a pair,

(iii) for all $\tau \in \Pi\left(E_{f}^{+}\right)$and all types $\tau^{\prime} \in \operatorname{subs}(\tau) \backslash\left\{\tau^{\prime}\right\}$, we have top $\left(\tau^{\prime}\right) \neq$ $\operatorname{top}(v)$,

(iv) for all terms $u \in \operatorname{Rec}\left(F_{f}, t\right), u \neq A x v$,

(v) $a \notin \operatorname{subs}(A x) \cup \operatorname{subs}(\operatorname{lhs}(R))$, 
(vi) $\operatorname{top}(v) \notin c t(A x)$, and

(vii) for all $\left\{s, s^{\prime}\right\} \in A x$, we have that vars $(s)=\operatorname{vars}\left(s^{\prime}\right)$.

Then $t \rho_{[a / v]}$ is $R$,Ax-normal and $f(t)=f\left(t \rho_{[a / v]}\right)$.

Proof. The first conjunct, namely $t \rho_{[a / v]}$ is $R, A x$-normal, follows from Lemma 23 . We now show that $f(t)=f\left(t \rho_{[a / v]}\right)$ by induction on the size of $t$.

- If $t$ is an atom then since $v$ is composed, we have $t \rho_{[a / v]}=t$. Thus we obtain $f(t)=f\left(t \rho_{[a / v]}\right)$.

- If $t=c\left(t_{1}, \ldots, t_{n}\right)$ for some $c \in \Sigma^{n}$ and $n \geq 1$, then let $f(p)=q$ be the pattern in $E_{f}^{+}$that is chosen for $t$. Let $p: \tau$. Then we have $\Gamma(t) \preccurlyeq \tau$. We show that $\Gamma\left(t \rho_{[a / v]}\right) \preccurlyeq \tau$.

Suppose there is a position $k$ such that $\left.t\right|_{k}={ }_{A x} v$. It is sufficient to show that $\Gamma\left(t[a]_{k}\right) \preccurlyeq \tau$. Let us consider two cases.

- If $k \in \operatorname{Pos}(\tau)$ then we have $\left.\Gamma\left(\left.t\right|_{k}\right) \preccurlyeq \tau\right|_{k}$. Since $\left.t\right|_{k}={ }_{A x} v$ and $v$ is composed, we derive that $t_{k}$ is composed and

$$
\operatorname{top}\left(t_{k}\right)=\operatorname{top}(v) .
$$

Note that $t \in \operatorname{Rec}\left(F_{f}, t\right)$. By assumption (iii), we have $t \neq_{A x} v$. Since $\left.t\right|_{k}=A_{A x} v$ and $t \neq_{A x} v$, we know that $k$ is not the root of $t$. That means $\left.\tau\right|_{k}$ is a strict subterm of $\tau$. Hence, by assumption (iii), we derive that $\operatorname{top}\left(\left.\tau\right|_{k}\right) \neq \operatorname{top}(v)$. This by (24) implies that $\operatorname{top}\left(\left.\tau\right|_{k}\right) \neq \operatorname{top}\left(t_{k}\right)$. Moreover, we know that $\left.t\right|_{k}$ is composed and $\left.\Gamma\left(\left.t\right|_{k}\right) \preccurlyeq \tau\right|_{k}$. Therefore, we must have that $\left.\tau\right|_{k}=m s g$ and obtain $\Gamma\left(t[a]_{k}\right) \preccurlyeq \tau$ as desired.

- If $k \notin \mathcal{P} o s(\tau)$ then there must be a position $k^{\prime}$ above $k$ such that $\left.\tau\right|_{k}=$ $m s g$. This also yields $\Gamma\left(t[a]_{k}\right) \preccurlyeq \tau$.

Hence, we have shown that $\Gamma\left(t \rho_{[a / v]}\right) \preccurlyeq \tau$. Similarly, it holds that whenever a pattern $p^{\prime}$ matches $t \rho_{[a / v]}$, it also matches $t$. This implies $t$ and $t \rho_{[a / v]}$ are abstracted under $f$ by the same clause. Let $\theta$ and $\theta^{\prime}$ be substitutions such that $t=p \theta$ and $t \rho_{[a / v]}=p \theta^{\prime}$. We perform a case distinction on $p$.

- $p=c\left(p_{1}, \ldots, p_{n}\right)$ for $c \in \Sigma^{n} \backslash\left\{\mathrm{C}_{\mathrm{Ax}} \cup \mathrm{C}_{\text {Key }}\right\}$. In this case, we have

$$
f\left(c\left(p_{1}, \ldots, p_{n}\right)\right)=\left\langle e_{1}, \ldots, e_{d}\right\rangle
$$

for some $d>0$ and for all $i \in \widetilde{d}, e_{i}$ is one of the following forms:

1. $e_{i}=c\left(\widehat{f}\left(\overline{q_{1}}\right), \ldots, \widehat{f}\left(\overline{q_{n}}\right)\right)$ such that

$$
\begin{aligned}
& \operatorname{set}\left(\overline{q_{j}}\right) \subseteq \operatorname{split}\left(p_{j}\right) \text { for all } j \in \widetilde{n}, c \neq\langle\cdot, \cdot\rangle, \text { and } \\
& c \in \mathrm{C}_{\mathrm{R}} \Rightarrow \overline{q_{n}}=\left[p_{n}\right] .
\end{aligned}
$$

2. $e_{i}=f(q)$ such that $q \in \operatorname{split}\left(p_{j}\right)$ for some $j \in \widetilde{n}$.

and it holds that $\forall j \in p p(c)$. split $\left(p_{j}\right) \subseteq Q(j)$ where

$$
\begin{gathered}
Q(j)=\bigcup\left\{\operatorname{set}\left(\overline{q_{j}}\right) \mid c\left(\widehat{f}\left(\overline{q_{1}}\right), \ldots, \widehat{f}\left(\overline{q_{n}}\right)\right) \in\left\{e_{1}, \ldots, e_{d}\right\}\right\} \\
\bigcup\left\{q \mid f(q) \in\left\{e_{1}, \ldots, e_{d}\right\}\right\} .
\end{gathered}
$$


Hence, we have

$$
\begin{aligned}
f(t) & =\left\langle e_{1} \theta, \ldots, e_{d} \theta\right\rangle, \\
f\left(t \rho_{[a / v]}\right) & =\left\langle e_{1} \theta^{\prime}, \ldots, e_{d} \theta^{\prime}\right\rangle .
\end{aligned}
$$

To see that $f\left(t \rho_{[a / v]}\right)=f(t)$, it is sufficient to show that $e_{i} \theta=e_{i} \theta^{\prime}$ for all $i \in \widetilde{d}$. Let $i \in \widetilde{d}$. We consider two cases.

$* e_{i}=c\left(\widehat{f}\left(\overline{q_{1}}\right), \ldots, \widehat{f}\left(\overline{q_{n}}\right)\right)$. To show that $e_{i} \theta=e_{i} \theta^{\prime}$, it is sufficient to show that $\widehat{f}\left(\overline{q_{j}} \theta\right)=\widehat{f}\left(\overline{q_{j}} \theta^{\prime}\right)$ for all $j^{\prime} \in \widetilde{n}$. Let $j \in \widetilde{n}$. Since $v$ is not a pair and the fact that $(p \theta) \rho_{[a / v]}=p \theta^{\prime}$, we have $(w \theta) \rho_{[a / v]}=w \theta^{\prime}$ for all $w \in \operatorname{set}\left(\overline{q_{j}}\right)$. Moreover, by assumption (iv), we have $w \theta \neq_{A x} v$. Note that $w \theta \in \operatorname{subs}(t) \backslash\{t\}$. Hence, by induction hypothesis, we have $f\left(w \theta^{\prime}\right)=f(w \theta)$. This yields $\widehat{f}\left(\overline{q_{j}} \theta\right)=\widehat{f}\left(\overline{q_{j}} \theta^{\prime}\right)$ as desired.

$* e_{i}=f(q)$ with $q \in \operatorname{split}\left(p_{j}\right)$ for some $j \in \widetilde{n}$. Since $(p \theta) \rho_{[a / v]}=p \theta^{\prime}$, we derive that $q \theta^{\prime}=(q \theta) \rho_{[a / v]}$. Moreover, $q \in \operatorname{split}\left(p_{j}\right)$ implies that $q \theta \in \operatorname{subs}(t) \backslash\{t\}$. By induction hypothesis, we know that $f\left(q \theta^{\prime}\right)=$ $f(q \theta)$. Therefore, we obtain $e_{i} \theta=e_{i} \theta^{\prime}$ as required.

- $p=c\left(p_{1}, \ldots, p_{n}\right)$ for $c \in \Sigma^{n} \cap\left(\mathrm{C}_{\mathrm{Ax}} \cup \mathrm{C}_{\mathrm{Key}}\right)$. In this case, we have

$$
\begin{aligned}
f(p) & =c\left(f\left(p_{1}\right), \ldots, f\left(p_{n}\right)\right) \\
f(t) & =c\left(f\left(t_{1}\right), \ldots, f\left(t_{n}\right)\right) \\
f\left(t \rho_{[a / v]}\right) & =c\left(f\left(t_{1} \rho_{[a / v]}\right), \ldots, f\left(t_{n} \rho_{[a / v]}\right)\right) .
\end{aligned}
$$

From assumption (iv), we have $t_{i} \neq_{A x} v$ for all $i \in \widetilde{n}$. Thus by the induction hypothesis, we have $f\left(t_{i}\right)=f\left(t_{i} \rho_{[a / v]}\right)$. Thus we obtain $f(t)=$ $f\left(t \rho_{[a / v]}\right)$ as required.

This completes the proof of the lemma.

In the following lemma, we introduce the notion of composite-preserving. We say that $f$ is composite-preserving if for all clauses $(f(p)=u) \in E_{f}$ such that $\operatorname{top}(p) \in \Sigma^{n}$ with $n \geq 1$, we have either (i) top $(q) \in \Sigma^{m} \backslash\{f\}$ with $m \geq 1$, or (ii) $q=f\left(q^{\prime}\right)$ for some term $q^{\prime}$ such that $\Gamma\left(q^{\prime}\right)$ is composed. Intuitively, $f$ is composite-preserving if it cannot produce a non-composed term from a composed one.

Lemma 29. Let $t$ be an $R, A x$-normal term and $f$ be composite-preserving and homomorphic for top $(t)$. Suppose that there exists a rewrite rule $l \rightarrow r \in R$ and a substitution $\sigma$ such that

(i) $f(t)={ }_{A x} l \sigma$,

(ii) $l$ is Ax-stable.

Then we have $l \rightarrow r \notin \mathrm{R} 3 *$.

Proof. We prove this lemma by contradiction. Suppose that $l \rightarrow r \in \mathrm{R}^{*}$ and $t=c\left(t_{1}, \ldots, t_{n}\right)$ for some $c \in \Sigma^{n}$. Since $f$ is homomorphic for $c$, we have

$$
f(t)=c\left(f\left(t_{1}\right), \ldots, f\left(t_{n}\right)\right) .
$$


By assumption (i), we obtain that $c\left(f\left(t_{1}\right), \ldots, f\left(t_{n}\right)\right)={ }_{A x} l \sigma$. Moreover, by assumption (ii), we derive that $l=c\left(u_{1}, \ldots, u_{n}\right)$ for some terms $u_{1}, \ldots, u_{n}$ and $f\left(t_{i}\right)={ }_{A x} u_{i} \sigma$ for all $i \in \tilde{n}$. We also know that $u_{i}$ is either a variable or a constant. Let us define the substitution $\sigma^{\prime}$ such that $\sigma=\left\{f\left(t_{i}\right) / u_{i} \mid i \in\right.$ $\tilde{n}$ and $u_{i}$ is a variable $\}$. We show that $t=l \sigma^{\prime}$. To see this, it is sufficient to show that $u_{i}$ is a constant implies $u_{i}=t_{i}$. Indeed, let $i \in \widetilde{n}$ such that $u_{i}$ is a constant. Since $f\left(t_{i}\right)={ }_{A x} u_{i} \sigma$, we derive that $f\left(t_{i}\right)=u_{i}$. This together with the assumption that $f$ is composite-preserving implies $t_{i}$ is not composed. Therefore, we have $t_{i}=u_{i}$. Hence, we have shown that $t=l \sigma^{\prime}$. This contradicts our assumption that $t$ is $R, A x$-normal and thus completes the proof of the lemma.

In the following lemma, we define the set of terms $\operatorname{Dec}(\sigma)$ for a given substitution $\sigma$ as follows.

$$
\operatorname{Dec}(\sigma)=\{t \mid t \in \operatorname{subs}(\operatorname{ran}(\sigma)) \wedge \operatorname{top}(t) \in \operatorname{top}(\operatorname{lhs}(R)) \wedge f(t) \text { is a redex }\}
$$

Lemma 30. Let $\phi \in \mathcal{L}_{P}$, and $(t r, t h, \sigma)$ be a reachable state of $P$. Suppose that

(i) $f$ is composite-preserving and homomorphic for top $(\operatorname{lhs}(R))$,

(ii) for all $\tau \in \Pi\left(E_{f}^{+}\right)$and all types $\tau^{\prime} \in \operatorname{subs}(\tau) \backslash\left\{\tau^{\prime}\right\}$, we have top $\left(\tau^{\prime}\right) \notin$ top $(\operatorname{lhs}(R))$,

(iii) for all terms $v \in \operatorname{subs}\left(\mathcal{M}_{P} \cup S e c_{\phi} \cup E q T e r m_{\phi}\right)$, we have $f(v)$ is $R$, Ax-stable,

(iv) EqTerm $_{\phi}$ is R,Ax-stable,

(v) $\mathcal{M}_{P} \cup \operatorname{Sec}_{\phi} \cup \operatorname{EqTerm} \operatorname{Te}_{\phi} \subseteq \operatorname{udom}\left(F_{f}\right)$,

(vi) $\langle\cdot, \cdot\rangle \notin \operatorname{top}(\operatorname{lhs}(R))$,

(vii) $l$ is Ax-stable for all $l \in l h s\left(\mathrm{R}^{*}\right)$,

(viii) $\operatorname{top}\left(\operatorname{lhs}\left(R \backslash \mathrm{R}^{*}\right)\right) \cap \operatorname{ct}(A x)=\emptyset$, and

(ix) for all $l \in$ lhs $(R)$ and all non-variable positions $p \in \mathcal{P}$ os $(l) \backslash\{\epsilon\}$, we have that top $\left(\left.l\right|_{p}\right) \notin$ top $(\operatorname{lhs}(R))$, and

(x) for all $\left\{s, s^{\prime}\right\} \in A x$, we have that $\operatorname{vars}(s)=\operatorname{vars}\left(s^{\prime}\right)$.

Then there is an $R, A x$-normal ground well-typed substitution $\sigma_{0}$ such that $f\left(\sigma_{0}\right)$ is $R, A x$-normal and the following holds:

$-\left|\operatorname{Dec}\left(\sigma_{0}\right)\right|<|\operatorname{Dec}(\sigma)|$,

- $\left(t r, t h, \sigma_{0}\right)$ is a reachable state in $P$, and

- if $(\mathrm{tr}, \mathrm{th}, \sigma) \not \models \phi$ then $\left(\mathrm{tr}, \mathrm{th}, \sigma_{0}\right) \not \models \phi$.

Proof. Without loss of generality, we assume that $\sigma$ is $R, A x$-normal. By assumption (vi) and Lemma 6 , we derive that $f(\sigma)$ is not $R, A x$-normal if and only if $\operatorname{Dec}(\sigma) \neq \emptyset$. Let $v_{0} \in \operatorname{Dec}(\sigma)$ and $a_{0}$ be a constant that does not occur in $\phi, \operatorname{ran}(\sigma), R$, and $A x$. First, we show that $\operatorname{top}\left(v_{0}\right) \cap \operatorname{ct}(A x)=\emptyset$. Since $f\left(v_{0}\right)$ is a redex, there exists a rewrite rule $l \rightarrow r \in R$ and a substitution $\theta$ such that $f\left(v_{0}\right)={ }_{A x} l \theta$. By assumptions (i),(vii) and Lemma 29, we derive that $l \rightarrow r \notin \mathrm{R} 3 *$. This by assumption (viii) implies that $\operatorname{top}(l) \cap \operatorname{ct}(A x)=\emptyset$. Moreover, by assumption (i), we have top $\left(v_{0}\right)=\operatorname{top}\left(f\left(v_{0}\right)\right)$. Since all equations $s \simeq s^{\prime}$ 
in $A x$ satisfy that $\operatorname{top}(s)=\operatorname{top}\left(s^{\prime}\right)$, we derive that $\operatorname{top}\left(f\left(v_{0}\right)\right)=\operatorname{top}(l \theta)$. As $l$ is not a variable, we also have $\operatorname{top}(l)=\operatorname{top}(l \theta)$. Therefore, we obtain that $\operatorname{top}\left(v_{0}\right)=\operatorname{top}(l)$. This yields $\operatorname{top}\left(v_{0}\right) \cap \operatorname{ct}(A x)=\emptyset$.

Second, we define $\sigma_{0}=\sigma \rho_{\left[a_{0} / v_{0}\right]}$ and show that $\sigma_{0}$ is well-typed. Note that $\sigma$ is well-typed by assumption. Let $X \in \operatorname{dom}\left(\sigma_{0}\right)$ and suppose that $\operatorname{subs}(X \sigma) \cap$ $\operatorname{dom}\left(\rho_{\left[a_{0} / v_{0}\right]}\right) \neq \emptyset$. Then $X \sigma$ is composed. Since $\sigma$ is well-typed, we must have $X: m s g$. Therefore, we have $\Gamma\left(\left(X \sigma_{0}\right) \downarrow_{R, A x}\right) \preccurlyeq \Gamma(X)$. Hence $\sigma_{0}$ is well-typed.

Third, we show that $\sigma_{0}$ is $R, A x$-normal. Let $X \in \operatorname{dom}\left(\sigma_{0}\right)$. Since $\sigma$ is $R, A x$-normal, so is $X \sigma$. By Lemma 23, we have $(X \sigma) \rho_{\left[a_{0} / v_{0}\right]}$ is $R, A x$-normal. Thus $X \sigma_{0}$ is $R, A x$-normal. Hence $\sigma_{0}$ is $R, A x$-normal.

We now show that $\left|\operatorname{Dec}\left(\sigma_{0}\right)\right|<|\operatorname{Dec}(\sigma)|$. For this purpose, it is sufficient to show that for all terms $t \in \operatorname{subs}(\operatorname{ran}(\sigma))$ such that $f(t)$ is $R, A x$-normal, we also have $f\left(t \rho_{\left[a_{0} / v_{0}\right]}\right)$ is $R, A x$-normal. Let $t \in \operatorname{subs}(\operatorname{ran}(\sigma))$ such that $f(t)$ is $R, A x$-normal. We claim that for all $u \in \operatorname{Rec}\left(F_{f}, t\right)$, it holds that $u \neq_{A x} v_{0}$. To see that, let us pick an arbitrary term $u \in \operatorname{Rec}\left(F_{f}, t\right)$. Since $f(t)$ is $R, A x$-normal, so is $f(u)$. Suppose that $u={ }_{A x} v_{0}$. By Proposition 3, we know that $f(u)={ }_{A x}$ $f\left(v_{0}\right)$. Since $f\left(v_{0}\right)$ is not $R, A x$-normal, neither is $f(u)$. This, together with $u \in \operatorname{Rec}\left(F_{f}, t\right)$, implies that $f(t)$ is not $R, A x$-normal which is a contradiction. Therefore, we must have $u \neq_{A x} v_{0}$. Hence, we have established that $u \neq_{A x} v_{0}$ for all $u \in \operatorname{Rec}\left(F_{f}, t\right)$. Thus by Lemma 28, we have $f(t)=f\left(t \rho_{\left[a_{0} / v_{0}\right]}\right)$. Since $f(t)$ is $R, A x$-normal, so is $f\left(t \rho_{\left[a_{0} / v_{0}\right]}\right)$. Hence, we have just proved that

$$
\left|\operatorname{Dec}\left(\sigma_{0}\right)\right|<|\operatorname{Dec}(\sigma)| \text {. }
$$

Next, we show reachability for $\sigma_{0}$, i.e., we need to show that $\left(t r, t h, \sigma_{0}\right)$ is reachable in $P$. We prove this by induction on the number $n$ of transitions leading to $(t r, t h, \sigma)$.

- Base case $(n=0)$ : Since $(\epsilon, t h, \sigma)$ is reachable, so is $\left(\epsilon, t h, \sigma_{0}\right)$.

- Inductive case $(n=k+1)$ : Suppose $\left(t r^{\prime}, t h^{\prime}, \sigma\right)$ is reachable in $k$ steps and there is a transition $\left(t r^{\prime}, t h^{\prime}, \sigma\right) \rightarrow(t r, t h, \sigma)$. By induction hypothesis, we have

$$
\left(t r^{\prime}, t h^{\prime}, \sigma\right) \text { is reachable in } P \text {. }
$$

We consider two non-trivial cases according to the rule $r$ that has been applied in step $k+1$.

- If $r=S E N D$ then it is obvious that $\left(t r^{\prime}, t h^{\prime}, \sigma_{0}\right) \rightarrow\left(t r, t h, \sigma_{0}\right)$. This by (26) yields that $\left(t r, t h, \sigma^{\prime}\right)$ is reachable in $P$.

- If $r=R E C V$ then there exists $i \in T I D$ and $R \in \operatorname{dom}(P)$ such that $t h^{\prime}(i)=(R, \operatorname{rcv}(u) \cdot t l), t r=t r^{\prime} \cdot(i, \operatorname{rcv}(u)), t h=t h^{\prime}[i \mapsto(R, t l)]$, and

$$
I K\left(t r^{\prime}\right) \sigma, I K_{0} \vdash_{E} u \sigma .
$$

Let $T=\operatorname{subs}\left(I K(t r) \cup S e c_{\phi} \cup E q \operatorname{Eerm}_{\phi}\right)$. We show the following result.

$$
\forall t^{\prime} \in T \backslash \operatorname{vars}(T) . t^{\prime} \sigma_{0} \neq_{A x} v_{0} .
$$

Suppose that it is not the case, then there is a term $t^{\prime} \in T$ such that $t^{\prime} \sigma={ }_{A x} v_{0}$. By Proposition 3, we have $f\left(t^{\prime} \sigma_{0}\right)={ }_{A x} f\left(v_{0}\right)$. By assumption 
(v), we can apply Theorem 1 and obtain $f\left(t^{\prime} \sigma_{0}\right)=f\left(t^{\prime}\right) f\left(\sigma_{0}\right)$. This by assumption (iii) implies that $f\left(t^{\prime}\right.$ sigma $\left._{0}\right)$ is $R, A x$-normal. This is a contradiction since $f\left(t^{\prime} \sigma_{0}\right)={ }_{A x} f\left(v_{0}\right)$ and $f\left(v_{0}\right)$ is not $R, A x$-normal. Hence, we have shown (27). By (27) and Lemma 27, we derive that

$$
I K\left(t r^{\prime}\right) \sigma_{0}, I K_{0} \vdash_{E} u \sigma_{0} .
$$

Thus the reachability of $\left(t r, t h, \sigma_{0}\right)$ in $P$ follows immediately.

Then, we show attack preservation for $\sigma_{0}$, i.e., we need to show that

$$
\forall \vartheta .(t r, t h, \sigma, \vartheta) \not \models \phi \Rightarrow\left(t r, t h, \sigma_{0}, \vartheta\right) \not \models \phi .
$$

We prove this by induction on $\phi$. It is enough to consider the following cases.

$$
\begin{aligned}
& \text { - } \phi \equiv m=m^{\prime} \text { or } \phi \equiv \neg\left(m=m^{\prime}\right) . \\
& (t r, t h, \sigma, \vartheta) \vDash m=m^{\prime} \\
& \Leftrightarrow m \sigma={ }_{E} m^{\prime} \sigma \\
& \Leftrightarrow(m \sigma) \rho_{\left[a_{0} / v_{0}\right]}={ }_{E}\left(m^{\prime} \sigma\right) \rho_{\left[a_{0} / v_{0}\right]} \text { by Lemmas } 25 \\
& \Leftrightarrow m\left(\sigma \rho_{\left[a_{0} / v_{0}\right]}\right)={ }_{E} m^{\prime}\left(\sigma \rho_{\left[a_{0} / v_{0}\right]}\right) \text { by }(27) \\
& \Leftrightarrow m \sigma_{0}={ }_{E} m \sigma_{0} \\
& \Leftrightarrow\left(t r, t h, \sigma_{0}, \vartheta\right) \vDash m=m^{\prime} \\
& \text { - } \phi \equiv \text { honest }(i, R) \text { or } \phi \equiv \neg \text { honest }(i, R) \text {. } \\
& (\operatorname{tr}, \operatorname{th}, \sigma, \vartheta) \vDash h o n e s t(i, R) \\
& \Leftrightarrow R^{\vartheta(i)} \sigma \in \mathcal{A}_{H} \\
& \Leftrightarrow R^{\vartheta(i)} \sigma^{\prime} \in \mathcal{A}_{H} \\
& \Leftrightarrow\left(\operatorname{tr}, t h, \sigma_{0}, \vartheta\right) \vDash h o n e s t(i, R) \\
& \text { since } R^{\vartheta(i)} \sigma=R^{\vartheta(i)} \sigma_{0} \\
& -\phi \equiv \operatorname{secret}(m) . \\
& \begin{aligned}
& (t r, t h, \sigma, \vartheta) \not \models \operatorname{secret}(m) \\
\Leftrightarrow & I K(t r) \sigma, I K_{0} \vdash_{E} m \sigma \\
\Rightarrow & I K(t r) \sigma_{0}, I K_{0} \vdash_{E} m \sigma_{0} \\
\Leftrightarrow & \left(t r, t h, \sigma_{0}, \vartheta\right) \not \models \operatorname{secret}(m)
\end{aligned} \\
& \text { by (27) and Lemma } 27
\end{aligned}
$$

The inductive cases are routine.

Lemma 31. Let $\phi \in \mathcal{L}_{P}$, and $(t r, t h, \sigma)$ be a reachable state of $P$. Suppose that

(i) $f$ is composite-preserving and homomorphic for top $($ lhs $(R))$,

(ii) for all $\tau \in \Pi\left(E_{f}^{+}\right)$and all types $\tau^{\prime} \in \operatorname{subs}(\tau) \backslash\left\{\tau^{\prime}\right\}$, we have top $\left(\tau^{\prime}\right) \notin$ top $(\operatorname{lhs}(R))$,

(iii) for all terms $v \in \operatorname{subs}\left(\mathcal{M}_{P} \cup S_{e c} \cup E q T e r m_{\phi}\right)$, we have $f(v)$ is $R, A x$-stable,

(iv) $\mathrm{EqTerm}_{\phi}$ is R,Ax-stable,

(v) $\mathcal{M}_{P} \cup \operatorname{Sec}_{\phi} \cup \operatorname{EqTerm}{ }_{\phi} \subseteq$ udom $\left(F_{f}\right)$,

(vi) $\langle\cdot, \cdot\rangle \notin \operatorname{top}(\operatorname{lhs}(R))$, 
(vii) $l$ is Ax-stable for all $l \in l h s\left(\mathrm{R}^{*}\right)$,

(viii) $\operatorname{top}\left(\operatorname{lhs}\left(R \backslash \mathrm{R}^{*}\right)\right) \cap \operatorname{ct}(A x)=\emptyset$, and

(ix) for all $l \in$ lhs $(R)$ and all non-variable positions $p \in \mathcal{P}$ os $(l) \backslash\{\epsilon\}$, we have that top $\left(\left.l\right|_{p}\right) \notin$ top $($ lhs $(R))$, and

(x) for all $\left\{s, s^{\prime}\right\} \in A x$, we have that $\operatorname{vars}(s)=\operatorname{vars}\left(s^{\prime}\right)$.

Then there is an $R, A x$-normal ground well-typed substitution $\sigma^{\prime}$ such that $f\left(\sigma^{\prime}\right)$ is $R, A x$-normal and the following holds:

- $\left(\right.$ tr, th, $\left.\sigma^{\prime}\right)$ is a reachable state in $P$, and

- if $($ tr, th, $\sigma) \not \models \phi$ then $\left(\right.$ tr, th,$\left.\sigma^{\prime}\right) \not \models \phi$.

Proof. By Lemma 30, we know that there is a sequence of $R, A x$-normal ground well-typed substitution $\sigma_{0}$ such that $|\operatorname{Dec}(\sigma)|>\left|\operatorname{Dec}\left(\sigma_{0}\right)\right|$ and

- $\left(t r, t h, \sigma_{0}\right)$ is a reachable state in $P$, and

- if $(t r, t h, \sigma) \not \models \phi$ then $\left(t r, t h, \sigma_{0}\right) \not \models \phi$.

We keep applying Lemma 30 to construct a sequence of $R, A x$-normal ground well-typed substitution $\sigma_{0}, \sigma_{1}, \ldots, \sigma_{n}$ for some $n \geq 0$ such that $\left|\operatorname{Dec}\left(\sigma_{n}\right)\right|=0$ and

- $\left(t r, t h, \sigma_{n}\right)$ is a reachable state in $P$, and

- if $(t r, t h, \sigma) \not \models \phi$ then $\left(t r, t h, \sigma_{n}\right) \not \models \phi$.

Since $\left|\operatorname{Dec}\left(\sigma_{n}\right)\right|=0$, we have that $f\left(\sigma_{k}\right)$ is $R, A x$-normal. By setting $\sigma^{\prime}=\sigma_{n}$, we complete the proof of the lemma.

We say that $f$ is constructor-exclusive for the function symbol $c$ if for all $(f(p)=$ $q) \in E_{f}$ we have $\operatorname{top}(q)=c$ implies $\operatorname{top}(p)=c$. In the following lemma, we prove that every ground abstracted term with top-level constructor $c$ can only be obtained by transforming a term of the same form provided that $f$ is compositepreserving and constructor-exclusive for $c$.

Lemma 32. Let $t$ be an $R, A x$-normal ground term and $c$ be a function symbol. Suppose that

(i) $f$ is composite-preserving,

(ii) $f$ constructor-exclusive for $c$.

Then top $(f(t))=c$ implies top $(t)=c$.

Proof. The conclusion is trivial if $t$ is an atom. Suppose that $t$ is composed. Then there exists the first pattern $(f(p)=q) \in E_{f}^{+}$such that $\Gamma(t) \preccurlyeq \Gamma(p)$ and $p \theta=t$ for some substitution $\theta$. We distinguish two cases:

- If $(f(p)=q) \in E_{f}^{0}$ then we have that top $(q)=t o p(p)=t o p(t)=g^{\prime}$. We also have $c=\operatorname{top}(f(t))=\operatorname{top}(q \theta)=\operatorname{top}(t)$. This yields top $(t)=c$ as required. 
- If $(f(p)=q) \in E_{f}$ then assumption (i), we obtain that

$$
\begin{aligned}
t o p(t) & =\operatorname{top}(p) \\
t o p(f(t)) & =\operatorname{top}(q) .
\end{aligned}
$$

Moreover, by assumption (ii), we derive that $\operatorname{top}(t)=\operatorname{top}(p)=\operatorname{top}(q)=c$.

This completes the proof of the lemma.

Lemma 33. Let $t, u$ be ground terms and suppose that

(i) $f$ is composite-preserving,

(ii) $f$ is constructor-exclusive for all $c \in c t(u)$, and

(iii) $f$ is homomorphic for $\operatorname{ct}(u)$.

Then $f(t)=u$ implies $t=u$.

Proof. We prove this lemma by induction on $u$.

- If $u$ is an atom then since $f(t)=u$ and assumption (i), we derive that $t$ must be an atom. Hence, we have $f(t)=t$ and thus obtain $t=u$ as required.

- If $u=c\left(u_{1}, \ldots, u_{n}\right)$ for $c \in \Sigma^{n}$ and some terms $u_{1}, \ldots, u_{n}$, then since $f(t)=$ $u$, we have $\operatorname{top}(f(t))=c$. By Lemma 32, we derive that there are terms $t_{1}, \ldots, t_{n}$ such that $t=c\left(t_{1}, \ldots, t_{n}\right)$. Moreover, by assumption (iii), we have $f(t)=c\left(f\left(t_{1}\right), \ldots, f\left(t_{n}\right)\right)$. Since $f(t)=u$, we obtain that $f\left(t_{i}\right)=u_{i}$ for all $i \in \widetilde{n}$. By induction hypothesis, we have that $t_{i}=u_{i}$ for all $i \in \widetilde{n}$. Therefore, we derive that $t=u$ as required.

This completes the proof of the lemma.

Lemma 34. Let $t, u$ be terms such that msg $\notin \Gamma(\operatorname{vars}(u))$ and $\sigma$ be an $R, A x$ normal ground well-typed substitution such that $f(\sigma)=u f(\sigma)$. Assume that the following holds:

(i) $f$ is composite-preserving,

(ii) for all positions $p \in \mathcal{P}$ os $(t) \cap \mathcal{P}$ os $(u)$ such that $\left.t\right|_{p}$ is a message variable, we have

(a) $f$ is constructor-exclusive for all $c \in c t\left(\left.u\right|_{p}\right)$, and

(b) $f$ is homomorphic for $\operatorname{ct}\left(\left.u\right|_{p}\right)$.

Then we have $\left.f(\sigma)\right|_{\text {vars }(t)}=\left.\sigma\right|_{\text {vars }(t)}$.

Proof. It is sufficient to show that $X f(\sigma)=X \sigma$ for all $X \in \operatorname{vars}(t)$ such that $X: m s g$. Let $X$ be a message variable at position $p$ in $t$. Since $t f(\sigma)=u f(\sigma)$, it is clear that $p \in \mathcal{P}$ os $(u)$. Moreover, we have $X f(\sigma)=\left.u\right|_{p} f(\sigma)$. Note that $X f(\sigma)=f(X \sigma)$. Hence, by Lemma 33, we derive that $X \sigma=\left.u\right|_{p} f(\sigma)$. This implies $X f(\sigma)=X \sigma$ as required.

Finally, we are in a position to state our criterion. 
Proposition 7. Let $\phi \in \mathcal{L}_{P}$ be a property formula, $(t, u) \in E q_{\phi}$ such that msg $\notin$ $\Gamma(\operatorname{vars}(u))$, and $(t r, t h, \sigma)$ be a reachable state of $P$ such that $\sigma$ is $R, A x$-normal and $(\mathrm{tr}, \mathrm{th}, \sigma) \not \models \phi$. Assume that the following holds:

(i) $f$ is composite-preserving and homomorphic for top $($ lhs $(R))$,

(ii) for all $\tau \in \Pi\left(E_{f}^{+}\right)$and all types $\tau^{\prime} \in \operatorname{subs}(\tau) \backslash\left\{\tau^{\prime}\right\}$, we have top $\left(\tau^{\prime}\right) \notin$ $\operatorname{top}(\operatorname{lh} s(R))$,

(iii) for all terms $v \in \operatorname{subs}\left(\mathcal{M}_{P} \cup S e c_{\phi} \cup E q T e r m_{\phi}\right)$, we have $f(v)$ is $R, A x$-stable,

(iv) EqTerm $_{\phi}$ is R,Ax-stable,

(v) $\mathcal{M}_{P} \cup \operatorname{Sec}_{\phi} \cup \operatorname{EqTerm}{ }_{\phi} \subseteq$ udom $\left(F_{f}\right)$,

(vi) $f(t)=t$ and $f(u)=u$,

(vii) $t$ and $u$ are $R, A x$-stable, and $u$ is Ax-stable,

(viii) for all positions $p \in \mathcal{P}$ os $(t) \cap \mathcal{P}$ os $(u)$ such that $t_{p}$ is a message variable, we have

(a) $f$ is constructor-exclusive for all $c \in \operatorname{ct}\left(\left.u\right|_{p}\right)$ and

(b) $f$ is homomorphic for $c t\left(\left.u\right|_{p}\right)$,

(ix) $\langle\cdot, \cdot\rangle \notin \operatorname{top}(\operatorname{lhs}(R))$,

(x) $l$ is Ax-stable for all $l \in \operatorname{lhs}\left(\mathrm{R}^{*}\right)$,

(xi) $\operatorname{top}\left(\operatorname{lhs}\left(R \backslash \mathrm{R}^{*}\right)\right) \cap \operatorname{ct}(A x)=\emptyset$,

(xii) for all $l \in$ lhs $(R)$ and all non-variable positions $p \in \mathcal{P}$ os $(l) \backslash\{\epsilon\}$, we have that top $\left(\left.l\right|_{p}\right) \notin$ top $(\operatorname{lhs}(R))$, and

(xiii) for all $\left\{s, s^{\prime}\right\} \in A x$, we have that vars $(s)=\operatorname{vars}\left(s^{\prime}\right)$.

Then there is an $R, A x$-normal ground substitution $\sigma^{\prime}$ such that $\left(t r, t h, \sigma^{\prime}\right) \not \models \phi$ and $f\left(\sigma^{\prime}\right)$ is R,Ax-normal. Furthermore, if $f\left(t \sigma^{\prime}\right)={ }_{E} f\left(u \sigma^{\prime}\right)$ then $t \sigma^{\prime}={ }_{E} u \sigma^{\prime}$.

Proof. By points (i)-(v) and (ix)-(xiii), we apply Lemma 31 and derive that there is a ground $R, A x$-normal substitution $\sigma^{\prime}$ such that $\left(t r, t h, \sigma^{\prime}\right) \not \models \phi$ and $f\left(\sigma^{\prime}\right)$ is $R, A x$-normal. Suppose that $f\left(t \sigma^{\prime}\right)=_{E} f\left(u \sigma^{\prime}\right)$. We need to show that $t \sigma^{\prime}={ }_{E} u \sigma^{\prime}$. By assumption (v) and Theorem 1, we know that $f(t \sigma)=f(t) f(\sigma)$ and $f(u \sigma)=f(u) f(\sigma)$. By assumption (vi), we derive that $t f(\sigma)={ }_{E} u f(\sigma)$. Therefore, we obtain that $(t f(\sigma)) \downarrow_{R, A x}={ }_{A x}(u f(\sigma)) \downarrow_{R, A x}$. This by assumption (iv) and the $R, A x$-normality of $f(\sigma)$ implies $t f(\sigma)={ }_{A x} u f(\sigma)$. By (viii), we know that $u f(\sigma)$ is $A x$-stable. Therefore, we derive that $t f(\sigma)=u f(\sigma)$. By Lemma 34, we have $\left.f(\sigma)\right|_{\text {vars }(t)}=\left.\sigma\right|_{\text {vars }(t)}$. Note that since $m s g \notin \Gamma(\operatorname{vars}(u))$, we also have that $\left.f(\sigma)\right|_{\operatorname{vars}(u)}=\left.\sigma\right|_{\operatorname{vars}(u)}$. Hence, we obtain $t \sigma=u \sigma$ which yields $t \sigma={ }_{E} u \sigma$.

In Proposition 7, checking conditions (iv), (vii), and (x) require that an $A x$ unification algorithm exists. The other conditions can be effective checked. Note that for the XOR theory in Example 2 and the Diffie-Hellman theory in Example 1, conditions (ix)-(xiii) are satisfied.

We now apply our criterion to justify condition $\mathcal{I}$ with respect to protocol $\mathrm{IKE}_{\mathrm{m}}$ and the typed abstraction specified in Example 6, and the property $\phi_{a}$ formalized in Example 4. 
Example 11. First, we recall the typed abstraction $F_{1}=\left(f_{1}, E_{1}\right)$ in Example 6 , where $E_{1}$ is defined by the equations:

$$
\begin{aligned}
f_{1}\left(\{|X, Y|\}_{Z}\right) & =\left\langle f_{1}(X), f_{1}(Y)\right\rangle \\
f_{1}\left(\operatorname{mac}\left(X_{1}, \ldots, X_{8}\right)\right) & =\operatorname{mac}\left(\widehat{f}_{1}\left(\left[X_{1}, X_{3}, X_{5}, X_{6}, X_{7}, X_{8}\right]\right)\right) \\
f_{1}\left(\operatorname{mac}\left(Y_{1}, \ldots, Y_{8}\right)\right) & =\operatorname{mac}\left(\widehat{f}_{1}\left(\left[Y_{1}, Y_{5}, Y_{6}, Y_{7}, Y_{8}\right]\right)\right) \\
f_{1}\left(\operatorname{kdf}\left(Z_{1}, \ldots, Z_{5}\right)\right) & =\operatorname{kdf}\left(f_{1}\left(Z_{3}\right)\right) \\
f_{1}(\operatorname{prf}(U, Z)) & =f_{1}(U) \\
f_{1}\left(\exp \left(U_{1}, U_{2}\right)\right) & =\exp \left(f_{1}\left(U_{1}\right), f_{1}\left(U_{2}\right)\right), \\
f_{1}\left(\operatorname{sh}\left(U_{1}, U_{2}\right)\right) & =\operatorname{sh}\left(f_{1}\left(U_{1}\right), f_{1}\left(U_{2}\right)\right), \\
f_{1}\left(\left\{\left|V_{1}, V_{2}\right|\right\}_{Z}\right) & =\left\langle f_{1}\left(V_{1}\right), f_{1}\left(V_{2}\right)\right\rangle \\
f_{1}\left(\left\langle U_{3}, U_{4}\right\rangle\right) & =\left\langle f_{1}\left(U_{3}\right), f_{1}\left(U_{4}\right)\right\rangle,
\end{aligned}
$$

and $V_{1}: \alpha, X_{3}: \gamma_{o}, Y_{3}:$ nonce, $Z_{3}: \exp (m s g, m s g), U: \operatorname{kdf}(m s g)$ and all remaining pattern variables are of type $m s g$.

Second, we check conditions in Proposition 7. Conditions (ix)-(xii) hold for the considered equational theory. Conditions (i), (ii), and (v) immediately follow from the specification of $E_{f}$. Conditions (iii) and (iv) are also satisfied, since no term in the set $\operatorname{subs}\left(\mathcal{M}_{\mathrm{IKE}_{\mathrm{m}}} \cup E q T e r m_{\phi_{a}}\right)$ contains a function symbol from top $(\operatorname{lhs}(R))$ and $f$ is constructor-preserving. To check the remaining conditions, note that this proposition is applied for the case that $u=\exp \left(g, x^{j}\right)$ and $t=G a^{k}$, where $j, k \in T I D$ and $x$ is a nonce. Hence, we have $f_{1}(t)=t$ and $f_{1}(u)=u$. Therefore, condition (vi) is satisfied. Note that $\left.t\right|_{p}$ is a variable if and only if $p=\epsilon$. Since $c t\left(\left.u\right|_{\epsilon}\right)=\operatorname{ct}(u)=\{\exp \}$. It is not hard to see that $f$ is constructorexclusive for exp and $f$ is homomorphic for exp. Therefore, condition (viii) also holds.

In practice, syntactic criteria I (Proposition 6) and II (Proposition 7) are sufficient for many relevant case studies, e.g., all case studies in this thesis including those in Section 4 can be justified using these criteria. For authentication properties that involve agreement on atoms or variables of simple types such as nonces or timestamps, criterion I is applicable. Compared to I, criterion II has a larger scope and can be applied for authentication properties that involves message variables. The complexity of checking criterion II mostly contributes to computing Ax-unifiers. Nevertheless, an Ax-unification algorithm required for this criterion needs not compute a complete set of most general Ax-unifiers. Computing an Ax-unifier of two given terms in the case that such a unifiers exists is adequate.

\section{F.3 Soundness conditions for $\mathrm{IKE}_{\mathrm{m}}$-to-IKE $\mathrm{m}_{\mathrm{m}}^{1}$ abstraction}

Here, we establish the soundness conditions for the abstraction $F_{1}=\left(f_{1}, E_{f_{1}}\right)$ in Example 6 with respect to the properties $\phi_{s}$ and $\phi_{a}$ expressed in Example 4. For simplicity, let $\phi$ represent both $\phi_{s}$ and $\phi_{a}$. We assume that $I K_{0}=I K_{0}^{\prime}=$ $\mathcal{A} \cup \mathcal{C} \cup \mathcal{F} \bullet \cup \bigcup_{a \in \mathcal{A}, b \in \mathcal{A}_{C}} \operatorname{sh}(a, b)$. We need to show that the conditions required for Theorem 4 hold. These conditions are: 
(i) $f_{1}\left(I K_{0}\right) \subseteq I K_{0}^{\prime}$,

(ii) $F_{1}$ is $R, A x$-closed and pattern-disjoint,

(iii) $\mathcal{M}_{\mathrm{IKE}_{\mathrm{m}}} \subseteq \operatorname{udom}\left(F_{f_{1}}\right) \cap \operatorname{rdom}\left(F_{f_{1}}\right)$,

(iv) $\operatorname{Sec}_{\phi} \cup E q T e r m_{\phi} \subseteq \operatorname{udom}\left(F_{f_{1}}\right) \cap \operatorname{rdom}\left(F_{f_{1}}\right)$,

(v) $F_{f_{1}}(t \sigma)={ }_{E} F_{f_{1}}(u \sigma)$ implies $t \sigma={ }_{E} u \sigma$ for all $(t, u) \in E q_{\phi}^{+}$and for all well-typed and $R, A x$-normal ground substitutions $\sigma$, and

(vi) $F_{f_{1}}(t)=F_{f_{1}}(u)$ implies $t=u$, for all $e(t) \in \operatorname{Evt} t_{\phi}^{+}$and $e(u) \in \operatorname{Evt}\left(\mathcal{M}_{\mathbb{K K E}_{\mathrm{m}}}\right)$.

Note that the last three conditions are an unfolding of condition (iv) in Theorem 4. To justify conditions (i)-(vi), we rely on the following observations.

(O1) $f_{1}\left(I K_{0}\right)=I K_{0}=I K_{0}^{\prime}$.

(O2) All terms in $\operatorname{subs}\left(\mathcal{M}_{\mathbb{I K E}_{\mathrm{m}}} \cup \operatorname{Sec}_{\phi} \cup \operatorname{EqTerm}_{\phi}\right)$ are abstracted using only clauses in $E_{f_{1}}$.

(O3) No term in $\operatorname{subs}\left(\mathcal{M}_{\mathbb{K K E}_{\mathrm{m}}} \cup \operatorname{Sec}_{\phi} \cup E q T e r m_{\phi}\right)$ contains reducible function symbols.

(O4) for all terms $t, u \in \mathcal{M}_{\mathrm{IKE}_{\mathrm{m}}}$ such that $t \neq u$, we have $F_{f_{1}}(t) \neq F_{f_{1}}(u)$.

Condition (i) follows from (O1). To see that $F_{f_{1}}$ is pattern-disjoint, note that except mac, each function symbol is handled by at most one clause in $E_{f_{1}}$. Moreover, the types of the mac patterns in the second and the third clauses, i.e.,

$$
\begin{aligned}
& \operatorname{mac}\left(m s g, m s g, \gamma_{o}, m s g, m s g, m s g, m s g, m s g\right) \text { and } \\
& \operatorname{mac}(m s g, m s g, \text { nonce, msg, msg, msg, msg, msg), }
\end{aligned}
$$

are disjoint. This shows that $F_{f_{1}}$ is pattern-disjoint. In Example 9, we use a syntactic criterion to show that $F_{f_{1}}$ is $R, A x$-closed. Hence condition (ii) holds. Condition (iii) holds by (O2) and (O3). Condition (iv) follows from (O2) and (O3). Condition (vi) holds by (O4). To justify condition (ii), note that we can rewrite the equality on the tuples in $\phi_{a}$ as a conjunction of equalities on the tuples' components. Since $f_{1}$ is the identity on atoms and variables, it suffices to establish condition (ii) for the two equalities of the form $X=g(a)$ with $X$ is of type $m s g$ and $a$ is an atom. We formally justify these cases in Example 11. 


\section{G Untyped protocol abstractions}

Typed protocol abstractions allows simplifying protocols by pulling fields out of an encryptions or removing fields in a hash. However, they do not allow removing a term, e.g., a hash or a variable, completely. In order to abstract a protocol extensively, we introduce untyped protocol abstractions which are complementary to typed ones. Informally, an untyped protocol abstraction is a function $f_{u}: \mathcal{T} \rightarrow$ $\mathcal{T} \cup\{$ nil $\}$ which we extend to events, event sequences, traces, and protocols. Let $P$ be a protocol, we define

(i) $f_{u}(e v(t))=e v\left(f_{u}(t)\right)$ for events $e v(t) \in \operatorname{Evt}(\mathcal{T})$.

(ii) for event sequences, $f(\epsilon)=\epsilon$ and $f_{u}(e \cdot t l)=f_{u}(t l)$ if $\operatorname{term}\left(f_{u}(e)\right)=$ nil and $f_{u}(e \cdot t l)=f_{u}(e) \cdot f_{u}(t l)$ otherwise; the lifting to traces is defined analogously.

(iii) $f(P)(X)=P(X)$ for all $X \in \operatorname{dom}(P)$ such that $P(X) \neq \epsilon$.

\section{G.1 Redundancy removal abstraction}

In this section, we discuss protocol abstractions which allow us to remove redundancies in protocol specifications. For instance, we can remove intruder-derivable terms or repeated occurrences of a term. We call these abstractions redundancy removal abstractions.

Definition 11. A function $r d: \mathcal{M}_{P} \rightarrow \mathcal{M} \cup\{$ nil $\}$ is a redundancy removal abstraction $R D_{P}$ for a protocol $P$ if, for all $R \in \operatorname{dom}(P)$, we have $\operatorname{rd}\left(I K_{0}\right) P(R)$ where the predicate $\operatorname{rd}(T) S$ is inductively defined by the following two rules (where $r d(t)$ is removed from the deducibility conditions if it equals nil).

$$
\overline{r d(T) \epsilon} \quad \frac{r d(T \cup\{t\}) r \quad T, \mathcal{V}_{\alpha}, r d(t) \vdash_{E} t \quad T, \mathcal{V}_{\alpha}, t \vdash_{E} r d(t)}{r d(T) e v(t) \cdot r}
$$

We define $r d\left(t^{\# i}\right)=r d(t)^{\# i}$ and lift rd over roles, traces, protocols, and thread pools as expected.

Next, we define our class of redundancy removal abstractions for protocols.

Definition 12 (Redundancy removal abstractions for protocols). Let $P=\left(\Gamma_{P}, S_{P}\right)$ be a protocol. The set of redundancy removal abstractions $R D_{P}$ for $P$ is defined by

$$
R D_{P}=\left\{r d: \mathcal{M}_{P} \rightarrow \mathcal{M} \mid \forall R \in \operatorname{dom}\left(S_{P}\right) . r d\left(\Gamma_{P}\right) I K_{0} S_{P}(R)\right\} .
$$

For all $r d \in R D_{P}, t \in \mathcal{M}_{P}$, and $i \in T I D$, we define $r d\left(t^{\# i}\right)=r d(t)^{\# i}$.

We overload the notation and use term(tr) to denote the set of terms occurring in trace $t r$. In the following theorem, we show reachability preservation for redundancy removal abstractions. 
Lemma 35. Let $P$ be a protocol and $r d \in R D_{P}$. Then, for all states $(t r, t h, \sigma)$ reachable in $P$, we have $I K(r d(t r)) \sigma, I K_{0} \vdash_{E}$ term $(t r) \sigma$.

Proof. We proceed by induction on the number $n$ of transitions leading to a state $(t r, t h, \sigma)$. The theorem trivially holds for base case $(n=0)$ where $t r$ is the empty trace.

For the inductive case $(n=k+1)$, we assume that $\left(t r^{\prime}, t h^{\prime}, \sigma\right)$ is reachable in $k$ steps and there is a transition $\left(t r^{\prime}, t h^{\prime}, \sigma\right) \rightarrow(t r, t h, \sigma)$. Suppose that this transition is performed by thread $i$. From the transition rules, we know that $t r=t r^{\prime} \cdot(i, e v(t))$ for some $e v \in\{\mathrm{snd}, \mathrm{rcv}\}$. By the induction hypothesis, we have

$$
I K\left(r d\left(t r^{\prime}\right)\right) \sigma, I K_{0} \vdash_{E} \operatorname{term}\left(t r^{\prime}\right) \sigma .
$$

Since it follows from the induction hypothesis that

$$
I K(r d(t r)) \sigma, I K_{0} \vdash_{E} \operatorname{term}\left(t r^{\prime}\right) \sigma
$$

Moreover, we have term $(t r)=\operatorname{term}\left(t r^{\prime}\right) \cup\{t\}$. Thus it is sufficient to show

$$
I K(r d(t r)) \sigma, I K_{0} \vdash_{E} t \sigma .
$$

We do this by case analysis on the rule that justifies transition $k+1$.

- Rule SEND. We have $r d(t r)=r d\left(t r^{\prime}\right) \cdot(i, \operatorname{snd}(r d(t)))$ and thus $I K(r d(t r))=$ $I K\left(r d\left(t r^{\prime}\right)\right) \cup\{r d(t)\}$ if $r d(t) \neq$ nil and $r d(t r)=r d\left(t r^{\prime}\right)$ otherwise. Hence, we can derive

$$
\begin{array}{rlr}
I K(r d(t r)) \sigma, I K_{0} \vdash_{E} I K\left(r d\left(t r^{\prime}\right)\right) \sigma, r d(t) \sigma, I K_{0} & \text { by above } \\
\vdash_{E} \operatorname{term}\left(t r^{\prime}\right) \sigma, r d(t) \sigma, I K_{0} & \text { by induction hyp. }
\end{array}
$$

Next, since the terms of all events preceding snd $(t)$ on $P(R)$ are contained in $\operatorname{term}\left(t r^{\prime}\right)$ and $r d \in R D_{P}$, we derive $I K_{0}, \operatorname{term}\left(t r^{\prime}\right), \mathcal{V}_{\alpha}, r d(t) \vdash_{E} t$. Instantiating this with $\sigma$ and observing that $\left(\mathcal{V}_{\alpha}\right) \sigma \subseteq \mathcal{A} \subseteq I K_{0}$ yields

$$
\operatorname{term}\left(t r^{\prime}\right) \sigma, r d(t) \sigma, I K_{0} \vdash_{E} t \sigma .
$$

Combining this with the derivation above yields the desired conclusion (29). - Rule $R E C V$. In this case we can reason as follows.

$$
\begin{array}{rlrl}
I K(r d(t r)) \sigma, I K_{0} & \vdash_{E} \operatorname{term}\left(t r^{\prime}\right) \sigma, I K_{0} & & \text { by induction hypothesis }(28) \\
& \vdash_{E} I K\left(t r^{\prime}\right) \sigma, I K_{0} & \text { since } I K(t r) \subseteq \operatorname{term}\left(t r^{\prime}\right) \\
& \vdash_{E} t \sigma & \text { by second premise of rule } R E C V
\end{array}
$$

This establishes (29) as required.

This concludes the proof of the lemma.

Proposition 8. Let $P$ be a protocol and $r d \in R D_{P}$. Suppose that $I K_{0} \subseteq I K_{0}^{\prime}$. Then, for all states $(t r, t h, \sigma)$ reachable in $P,(r d(t r), r d(t h), \sigma)$ is a reachable state of $r d(P)$. 
Proof. We proceed by induction on the number $n$ of transitions leading to a state $(t r, t h, \sigma)$. The theorem trivially holds for base case $(n=0)$ where $t r$ is the empty trace. For the inductive case $(n=k+1)$, we assume that $\left(t r^{\prime}, t h^{\prime}, \sigma\right)$ is reachable in $k$ steps and there is a transition $\left(t r^{\prime}, t h^{\prime}, \sigma\right) \rightarrow(t r, t h, \sigma)$. Suppose that this transition is performed by thread $i$. From the transition rules, we know that we have $t h^{\prime}(i)=(R, e v(t) \cdot t l)$ where $R \in \operatorname{dom}(P), e v \in$ \{snd, rcv $\}$, and $t l$ is a suffix of the role $P(R)^{\# i}$. We also have $t r=t r^{\prime} \cdot(i, e v(t))$ and $t h=t h^{\prime}[i \mapsto t l]$.

By the induction hypothesis, we have $\left(r d\left(t r^{\prime}\right), r d\left(t h^{\prime}\right), \sigma\right)$ is a reachable state of $r d(P)$. If $r d(t)=$ nil then we are done, since $r d\left(t r^{\prime}\right)=r d(t r)$ and $r d\left(t h^{\prime}\right)=$ $r d(t h)$. Otherwise, we have $r d(t) \neq$ nil. In this case, it is sufficient to show that $r d(P)$ has a transition

$$
\left(r d\left(t r^{\prime}\right), r d\left(t h^{\prime}\right), \sigma\right) \rightarrow(r d(t r), r d(t h), \sigma) .
$$

We proceed by case distinction on the rule applied to justify step $k+1$ of $P$.

- SEND rule. The rule's premise requires $t h^{\prime}(i)=(R$, snd $(t) \cdot t l)$. Hence, by the definition of $r d\left(t h^{\prime}\right)$, we have $r d\left(t h^{\prime}\right)(i)=(R, e v(r d(t)) \cdot r d(t l))$. Moreover, we have $r d(t r)=r d\left(t r^{\prime}\right) \cdot(i$, snd $(r d(t)))$ and $r d(t h)=r d\left(t h^{\prime}\right)[i \mapsto r d(t l)]$, which by the $S E N D$ rule justifies the transition above.

- $R E C V$ rule. This rule's premises require that $t h^{\prime}(i)=(R, \operatorname{rcv}(t) \cdot t l)$ and

$$
I K\left(t r^{\prime}\right) \sigma, I K_{0} \vdash_{E} t \sigma .
$$

The rule's conclusion implies that $t r=t r^{\prime} \cdot(i, \operatorname{rcv}(t))$ and $t h=t h^{\prime}[i \mapsto t l]$. In order to apply the $R E C V$ rule in the state $\left(r d\left(t r^{\prime}\right), r d\left(t h^{\prime}\right), \sigma\right)$ two premises must be satisfied: first, $r d\left(t h^{\prime}\right)(i)=(R, \operatorname{rcv}(r d(e)) \cdot r d(t l))$, which holds by the definition of $r d\left(t h^{\prime}\right)$, and, second,

$$
I K\left(r d\left(t r^{\prime}\right)\right) \sigma, I K_{0}^{\prime} \vdash_{E} r d(t) \sigma,
$$

which we show now. Since $r d \in R D_{P}$ and term $\left(t r^{\prime}\right)$ contains the terms of all events preceding $\operatorname{rcv}(t)$ on $P(R)$, we have $I K_{0}$, term $\left(t r^{\prime}\right), \mathcal{V}_{\alpha}, t \vdash_{E} r d(t)$. Noting that $\operatorname{term}(\operatorname{tr})=\operatorname{term}\left(\operatorname{tr}^{\prime}\right) \cup\{t\}$ and $\left(\mathcal{V}_{\alpha}\right) \sigma \subseteq \mathcal{A} \subseteq I K_{0}$ we derive

$$
\operatorname{term}(\operatorname{tr}) \sigma, I K_{0} \vdash_{E} \operatorname{rd}(t) \sigma .
$$

Moreover, from Lemma 35, we have $I K(r d(t r)) \sigma, I K_{0} \vdash_{E} \operatorname{term}(t r) \sigma$. Combining these facts with the observation that $I K(r d(t r))=I K\left(r d\left(t r^{\prime}\right)\right)$ and the assumption that $I K_{0} \subseteq I K_{0}^{\prime}$, we obtain (30) as required.

This completes the proof of the theorem.

Next, we extend $r d$ to formulas $\phi \in \mathcal{L}_{P}$ as follows:

$$
\begin{aligned}
& r d\left(\left(i=i^{\prime}\right)\right)=\left(i=i^{\prime}\right) \\
& r d\left(\left(m=m^{\prime}\right)\right)=\left(m=m^{\prime}\right) \\
& \operatorname{rd}(\operatorname{role}(i, R))=\operatorname{role}(i, R) \\
& \operatorname{rd}(\text { honest }(i, R))=\operatorname{honest}(i, R) \\
& r d(\operatorname{steps}(i, e))=\operatorname{steps}(i, r d(e)) \\
& r d\left((i, e) \prec\left(j, e^{\prime}\right)\right)=(i, r d(e)) \prec\left(j, r d\left(e^{\prime}\right)\right) \\
& r d(\operatorname{secret}(m))=\operatorname{secret}(m) \\
& r d(\neg A)=\neg r d(A) \\
& r d\left(\phi_{1} \wedge \phi_{2}\right)=r d\left(\phi_{1}\right) \wedge r d\left(\phi_{2}\right) \\
& r d\left(\phi_{1} \vee \phi_{2}\right)=r d\left(\phi_{1}\right) \vee r d\left(\phi_{2}\right) \\
& r d\left(\forall i . \phi^{\prime}\right)=\forall i . r d\left(\phi^{\prime}\right) \\
& r d\left(\exists i . \phi^{\prime}\right)=\exists i . r d\left(\phi^{\prime}\right)
\end{aligned}
$$


Let $r d \in R D_{P}$. In the following, we define the notion of $(P, r d)$-safe formulas for which the attack preservation holds.

Definition $13((P, r d)$-safe formulas). Let $P$ be a protocol and $r d$ a redundancy abstraction for $P$. A formula $\phi$ is $(P, r d)$-safe if

1. $r d(t)=r d(u)$ implies $t=u$, for all $e(t) \in \operatorname{Evt}_{\phi}^{+}$and $e(u) \in \operatorname{Evt}\left(\mathcal{M}_{P}\right)$,

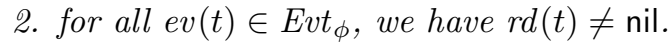

Theorem 6 (Soundness for redundancy removal abstractions). Let $P$ be a protocol, $\phi \in \mathcal{L}_{P}$, and $r d \in R D_{P}$ be a $(P, r d)$-safe formula. Suppose that $I K_{0} \subseteq I K_{0}^{\prime}$. Then, for all states $(t r, t h, \sigma)$ reachable in $P$, we have

1. $(r d(t r), r d(t h), \sigma)$ is reachable in $r d(P)$ and

2. $(\operatorname{tr}, t h, \sigma) \not \models \phi$ implies $(r d(t r), r d(t h), \sigma) \not \models r d(\phi)$.

Proof. By Proposition 8, we have that $(r d(t r), r d(t h), \sigma)$ is reachable in $r d(P)$. It remains to show that

$$
\forall \vartheta .(t r, t h, \sigma, \vartheta) \not \models \phi \Rightarrow(r d(t r), r d(t h), \sigma) \not \models r d(\phi) .
$$

We proceed by induction on the structure of $\phi$ and consider the following cases.

- $\phi \equiv m=m^{\prime}$ or $\phi \equiv \neg\left(m=m^{\prime}\right)$.

$$
\begin{aligned}
& (t r, t h, \sigma, \vartheta) \vDash m={ }_{E} m^{\prime} \\
\Leftrightarrow & m \sigma={ }_{E} m^{\prime} \sigma \\
\Leftrightarrow & (r d(t r), r d(t h), \sigma, \vartheta) \vDash r d\left(\left(m=m^{\prime}\right)\right)
\end{aligned}
$$

$-\phi=\operatorname{secret}(m)$.

$$
\begin{aligned}
& (\operatorname{tr}, t h, \sigma, \vartheta) \not \models \operatorname{secret}(m) \\
& \Leftrightarrow I K(t r) \sigma, I K_{0} \vdash_{E} m \sigma \\
& \Rightarrow I K(r d(t r)) \sigma, I K_{0} \vdash_{E} m \sigma \quad \text { by Lemma } 35 \\
& \Leftrightarrow(r d(t r), r d(t h), \sigma, \vartheta) \not \models r d(\operatorname{secret}(m))
\end{aligned}
$$

The remaining cases are routine. This completes the proof of the theorem. 


\section{G.2 Variable removal abstractions}

Our typed abstractions do not allow us to remove message variables. This is because our framework heavily relies on the substitution property, which fails to hold for the removal of variables. In this section, we establish a soundness result for variable removal abstractions separately for a subclass of protocols, namely well-formed protocols.

Definition 14. A protocol $P$ is well-formed if all non-agent variables first occur in receive events, i.e., for all events $e$ in a role $P(R)$ and all variables $X \in$ vars $($ term $(e))$ such that $\Gamma(X) \neq \alpha$, there is an event $\operatorname{rcv}(t)$ in $P(R)$ such that $\operatorname{rcv}(t)$ equals or precedes e in $P(R)$ and $X \in \operatorname{vars}(t)$.

Below we identify some conditions under which the set of messages the intruder can derive (and hence any potential attack) is preserved by variable removal. Before stating these assumptions, we introduce some auxiliary definitions.

First, we extend the notion of clearness to a set of terms $T$ as expected, i.e., $T$ is clear in a term $t$ if every term in $T$ is. We also say that $T$ is clear in a set of terms $T^{\prime}$ if every $t \in T$ is clear in every $t^{\prime} \in T^{\prime}$. In the following lemma, we abuse the notation and use vars(tr) to denote the set of variables occurring in tr.

Lemma 36. Let $P$ be a well-formed protocol and $(t r, t h, \sigma)$ be a reachable state of $P$. Let $V \subseteq \mathcal{V}$ be a set of variables such that $V$ is clear in $\mathcal{M}_{P}$. Then we have that $\operatorname{rem}_{V}(I K(t r)) \sigma, I K_{0} \vdash_{E}\left(I K(t r) \cup\left(\operatorname{vars}(t r) \cap V^{\mathrm{b}}\right)\right) \sigma$.

Proof. We proceed by induction on $t r$. For the base case, $t r=\epsilon$, the lemma holds trivially. For the inductive step, suppose $\left(t r^{\prime}, t h^{\prime}, \sigma\right)$ is reachable in $P$ and there is a transition $\left(t r^{\prime}, t h^{\prime}, \sigma\right) \rightarrow(t r, t h, \sigma)$ such that $t r=t r^{\prime} \cdot(i, e v(t))$ for some $i \in T I D$ and some term $t$. By induction hypothesis, we have

$$
\operatorname{rem}_{V}\left(I K\left(t r^{\prime}\right)\right) \sigma, I K_{0} \vdash_{E}\left(I K\left(t r^{\prime}\right) \cup\left(\operatorname{vars}\left(t r^{\prime}\right) \cap V^{b}\right)\right) \sigma .
$$

and we have to show $\operatorname{rem}_{V}(I K(t r)) \sigma, I K_{0} \vdash_{E}\left(I K(\operatorname{tr}) \cup\left(\operatorname{vars}(t r) \cap V^{b}\right)\right) \sigma$. We reason by a case distinction on the rule $r$ that has been applied in the last step.

- If $r=R E C V$ then we have that $I K\left(t r^{\prime}\right)=I K(t r)$. Thus by induction hypothesis, we have $\operatorname{rem}_{V}(I K(t r)) \sigma, I K_{0} \vdash_{E} I K(t r) \sigma$. Therefore, it remains to show that $\operatorname{rem}_{V}(I K(\operatorname{tr})) \sigma, I K_{0} \vdash_{E}\left(\operatorname{vars}(\operatorname{tr}) \cap V^{\mathrm{b}}\right) \sigma$.

Note that $t r=t r^{\prime} \cdot(i, \operatorname{rcv}(t))$. If vars $(t) \cap V^{b} \subseteq \operatorname{vars}\left(\operatorname{tr}^{\prime}\right)$ then vars $(t r) \cap$ $V^{b}=\operatorname{vars}\left(\operatorname{tr}^{\prime}\right) \cap V^{b}$ and the conclusion follows directly from the induction hypothesis. Otherwise, let $X^{i} \in\left(\operatorname{vars}(t) \cap V^{b}\right) \backslash \operatorname{vars}\left(t r^{\prime}\right)$. Given the induction hypothesis, it is sufficient to establish $\operatorname{rem}_{V}(I K(t r)) \sigma, I K_{0} \vdash_{E} X^{i} \sigma$.

By the premises of the $R E C V$ rule, we know that $I K\left(t r^{\prime}\right) \sigma, I K_{0} \vdash_{E} t \sigma$. Since $V$ is clear in $t$, we also have

$$
I K\left(t r^{\prime}\right) \sigma, I K_{0} \vdash_{E} X^{i} \sigma
$$

Since $V$ is clear in $I K\left(t r^{\prime}\right)$, we have

$$
I K_{0},\left(V^{\mathrm{b}} \cap \operatorname{vars}\left(I K\left(t r^{\prime}\right)\right)\right), \operatorname{rem}_{V}\left(I K\left(t r^{\prime}\right)\right) \vdash_{E} I K\left(t r^{\prime}\right) .
$$


By instantiating this with $\sigma$ and using the fact that $\operatorname{vars}\left(I K\left(t r^{\prime}\right)\right) \subseteq \operatorname{vars}\left(t r^{\prime}\right)$, we obtain

$$
I K_{0},\left(V^{b} \cap \operatorname{vars}\left(t r^{\prime}\right)\right) \sigma, \operatorname{rem}_{V}\left(I K\left(t r^{\prime}\right)\right) \sigma \vdash_{E} I K\left(t r^{\prime}\right) \sigma
$$

Together with the induction hypothesis and $I K(t r)=I K\left(t r^{\prime}\right)$, we derive

$$
\operatorname{rem}_{V}(I K(t r)) \sigma, I K_{0} \vdash_{E} I K\left(t r^{\prime}\right) \sigma .
$$

Combining this with (31), we obtain $\operatorname{rem}_{V}(I K(t r)) \sigma, I K_{0} \vdash_{E} X^{i} \sigma$ as required.

- If $r=S E N D$ then we have $t r=t r^{\prime} \cdot(i, \operatorname{snd}(t))$. Thus, we have that $I K(t r)=$ $I K\left(t r^{\prime}\right) \cup\{t\}$. By the well-formedness of $P$, we have $\operatorname{vars}(t r)=\operatorname{vars}\left(t r^{\prime}\right)$. Hence, it follows from the induction hypothesis that

$$
\operatorname{rem}_{V}(I K(t r)) \sigma, I K_{0} \vdash_{E}\left(\operatorname{vars}(t r) \cap V^{b}\right) \sigma .
$$

We are left to show that $\operatorname{rem}_{V}(I K(t r)) \sigma, I K_{0} \vdash_{E} I K(t r) \sigma$. Since $V$ is clear in $t$, we obtain

$$
I K_{0},\left(V^{b} \cap \operatorname{vars}(t)\right) \sigma, \operatorname{rem}_{V}(t) \sigma \vdash_{E} t \sigma .
$$

Since $\operatorname{vars}(t) \subseteq \operatorname{vars}(t r)$. By (32), we have

$$
\operatorname{rem}_{V}(I K(t r)) \sigma, I K_{0} \vdash_{E}\left(\operatorname{vars}(t) \cap V^{b}\right) \sigma .
$$

Together with (34) and (33), we derive that

$$
\operatorname{rem}_{V}(I K(t r)) \sigma, I K_{0}, r e m_{V}(t) \sigma \vdash_{E} t \sigma .
$$

Since $t \in I K(t r)$, we have that $\operatorname{rem}_{V}(t) \sigma \in \operatorname{rem}_{V}(I K(t r)) \sigma$. Hence, we obtain that

$$
\operatorname{rem}_{V}(I K(t r)) \sigma, I K_{0} \vdash_{E} t \sigma
$$

By induction hypothesis, we have $r e m_{V}\left(I K\left(t r^{\prime}\right) \sigma\right), I K_{0} \vdash_{E} I K\left(t r^{\prime}\right) \sigma$. Hence, we derive that $\operatorname{rem}_{V}(I K(t r)) \sigma, I K_{0} \vdash_{E} I K(t r) \sigma$ as required.

This completes the proof of the lemma.

Proposition 9. Let $P$ be a well-formed protocol. Suppose $V$ is a set of variables and $u$ a term such that $V$ is clear in $\mathcal{M}_{P}$. Suppose $(t r, t h, \sigma)$ is a reachable state of $P$. Then $I K(t r) \sigma, I K_{0} \vdash_{E} u \sigma$ implies $I K\left(\operatorname{rem}_{V}(t r)\right) \sigma, I K_{0} \vdash_{E} \operatorname{rem}_{V}(u) \sigma$.

Proof. We derive

$$
\begin{array}{rlr}
I K\left(\operatorname{rem}_{V}(t r)\right) \sigma, I K_{0} & \vdash_{E} \operatorname{rem}_{V}\left(I K\left(t r^{\prime}\right)\right) \sigma, I K_{0} \\
& \vdash_{E} I K(t r) \sigma, I K_{0} & \text { by Lemma } 36 \\
& \vdash_{E} u \sigma, I K_{0} & \text { by assumption } \\
& \vdash_{E} \operatorname{rem}_{V}(u) \sigma &
\end{array}
$$

The last step follows the assumption that $V$ is clear in $\mathcal{M}_{P}$. 
The following theorem states the reachability preservation result for variable removal abstractions.

Theorem 7. Let $P$ be a well-formed protocol. Suppose $I K_{0} \subseteq I K_{0}^{\prime}$ and $V \subseteq \mathcal{V}$ is a set of variables such that

1. $V$ is clear in all terms $t$ occurring in receive events in $P$,

2. $V$ is clear in $\mathcal{M}_{P}$.

Let $(t r, t h, \sigma)$ be reachable in $P$. Then we have that $\left(\operatorname{rem}_{V}(t r), r e m_{T}(t h), \sigma\right)$ is reachable in $\operatorname{rem}_{T}(P)$.

Proof. We prove the reachability of the state $\left(\operatorname{rem}_{V}(t r), \operatorname{rem}_{V}(t h), \sigma\right)$ by induction on the number $n$ of transitions leading to a state $(t r, t h, \sigma)$. The theorem holds trivially for the empty trace $(n=0)$. For the inductive case $(n=$ $k+1)$, assume that $\left(t r^{\prime}, t h^{\prime}, \sigma\right)$ is reachable in $k$ steps and there is a transition $\left(t r^{\prime}, t h^{\prime}, \sigma\right) \rightarrow(t r, t h, \sigma)$. By induction hypothesis, state $\left(r e m_{V}\left(t r^{\prime}\right), r e m_{V}\left(t h^{\prime}\right), \sigma\right)$ is reachable in $\operatorname{rem}_{V}(P)$.

We distinguish two cases according to the rule $r$ used to justify the step $k+1$ in $P$. We first treat the case of the receive rule $(r=R E C V)$. The rule's premises require that there are $i \in T I D, R \in \operatorname{dom}(P)$, and a suffix $t l$ of the role $P(R)^{\# i}$ such that

$$
t h^{\prime}(i)=(R, \operatorname{rcv}(t) \cdot t l) \quad \text { and } \quad I K\left(t r^{\prime}\right) \sigma, I K_{0} \vdash t \sigma
$$

The rule's conclusion implies that $t r=t r^{\prime} \cdot(i, \operatorname{rcv}(t))$ and $t h=t h^{\prime}[i \mapsto t l]$. We consider two cases.

- If $\operatorname{rem}_{V}(t)=$ nil then we have that

$$
\begin{aligned}
\operatorname{rem}_{V}(t r) & =\operatorname{rem}_{V}\left(t r^{\prime}\right), \\
\operatorname{rem}_{V}(t h) & =\operatorname{rem}_{V}\left(t h^{\prime}\right) .
\end{aligned}
$$

Hence, we conclude that $\left(r e m_{V}(t r), r e m_{V}(t h), \sigma\right)$ is reachable in $\operatorname{rem}_{V}(P)$ by the induction hypothesis.

- If $\operatorname{rem}_{V}(t) \neq$ nil then we show that $\operatorname{rem}_{V}(P)$ has a transition

$$
\left(\operatorname{rem}_{V}\left(t r^{\prime}\right), \operatorname{rem}_{V}\left(t h^{\prime}\right), \sigma\right) \rightarrow\left(r e m_{V}(t r), r e m_{V}(t h), \sigma\right) .
$$

In order to apply the $R E C V$ rule in the state $\left(r e m_{V}\left(t r^{\prime}\right), r e m_{V}\left(t h^{\prime}\right), \sigma\right)$ the following two premises must be satisfied:

$$
\begin{aligned}
& \operatorname{rem}_{V}\left(t h^{\prime}\right)(i)=\left(R, \operatorname{rcv}\left(\operatorname{rem}_{V}(t)\right) \cdot \operatorname{rem}_{V}(t l)\right), \text { and } \\
& \left.I K\left(\operatorname{rem}_{V}\left(t r^{\prime}\right)\right)\right) \sigma, I K_{0}^{\prime} \vdash_{E} \operatorname{rem}_{V}(t) \sigma .
\end{aligned}
$$

The first premise holds by application of $r e m_{T}$ to $t h^{\prime}$. The second one follows from Proposition 9 and the assumption that $I K_{0} \subseteq I K_{0}^{\prime}$. The successor state in the conclusion of the $R E C V$ rule is

$$
\left(\operatorname{rem}_{V}\left(\operatorname{tr}^{\prime}\right) \cdot\left(i, \operatorname{rcv}\left(r e m_{V}(t)\right)\right), \operatorname{rem}_{V}\left(t h^{\prime}\right)\left[i \mapsto\left(R, \operatorname{rem}_{V}(t l)\right)\right], \sigma\right),
$$

which is identical to the state $\left(\operatorname{rem}_{V}(t r), r e m_{V}(t h), \sigma\right)$, whose reachability in $\operatorname{rem}_{V}(P)$ we have hereby established. 
The case of the send rule $(r=S E N D)$ is similar but simpler, since the deducibility condition falls away. This completes the proof of the theorem.

Definition 15 ( $\left(P, r^{r e m_{T}}\right)$-safe formulas). Let $P$ be a protocol and let $V \subseteq \mathcal{V}$ be a set of variables. A formula $\phi \in \mathcal{L}_{P}$ is $\left(P\right.$, rem $\left._{T}\right)$-safe if and only if the following holds.

1. for all $\left(m, m^{\prime}\right) \in E q_{\phi}$, we have vars $\left(\left\{m, m^{\prime}\right\}\right) \cap V^{\sharp}=\emptyset$,

2. for all terms $t \in S e c_{\phi}$, we have vars $(t) \cap V^{\sharp}=\emptyset$,

3. for all events $e(t) \in E v t_{\phi}$, we have $\operatorname{rem}_{V}(t) \neq$ nil, and

4. $\operatorname{rem}_{V}(m)=\operatorname{rem}_{V}\left(m^{\prime}\right)$ implies $m=m^{\prime}$ for all $s(m) \in \operatorname{Evt}_{\phi}^{+}$and $s\left(m^{\prime}\right) \in$ $\operatorname{Evt}\left(\mathcal{M}_{P}\right)$.

We now state our soundness theorem as follows.

Theorem 8 (Soundness for variable removal abstractions). Let $P$ be a well-formed protocol, $V \subseteq \mathcal{V}$ is a set of variables that is clear in $\mathcal{M}_{P}$. Let $\phi \in$ $\mathcal{L}_{P}$ be a $\left(P\right.$, rem $\left._{T}\right)$-safe formula and $(t r, t h, \sigma)$ be reachable in $P$. Suppose that $I K_{0} \subseteq I K_{0}^{\prime}$. Then we have that $\left(\operatorname{rem}_{V}(t r), r e m_{V}(t h), \sigma\right)$ is a reachable state in $\operatorname{rem}_{V}(P)$. Moreover, if $(\mathrm{tr}, \mathrm{th}, \sigma) \not \models \phi$ then $\left(\operatorname{rem}_{V}(\mathrm{tr}), \mathrm{rem}_{V}(\mathrm{th}), \sigma\right) \not \models \phi$.

Proof. By Theorem 7, we have that $\left(r e m_{V}(t r), r e m_{V}(t h), \sigma\right)$ is a reachable state in $\operatorname{rem}_{V}(P)$. It remains to show that

$$
\forall \vartheta .(\operatorname{tr}, t h, \sigma, \vartheta) \not \models \phi \Rightarrow\left(\operatorname{rem}_{T}(\operatorname{tr}), \operatorname{rem}_{T}(t h), \sigma\right) \not \models \operatorname{rem}_{T}(\phi) .
$$

We proceed by induction on the structure of $\phi$ and consider the following nontrivial cases.

$$
\begin{aligned}
& \text { - } \phi \equiv m=m^{\prime} \text { or } \phi \equiv \neg\left(m=m^{\prime}\right) . \\
& (t r, t h, \sigma, \vartheta) \vDash m=m^{\prime} \\
& \Leftrightarrow m \sigma={ }_{E} m^{\prime} \sigma \\
& \Leftrightarrow \operatorname{rem}_{T}(m) \sigma={ }_{E} \operatorname{rem}_{T}\left(m^{\prime}\right) \sigma \quad \text { (since } \phi \text { is }\left(P, V, r e m_{T}\right) \text {-safe) } \\
& \Leftrightarrow\left(\operatorname{rem}_{T}(t r), \operatorname{rem}_{T}(t h), \sigma, \vartheta\right) \vDash \operatorname{rem}_{T}(m)=\operatorname{rem}_{T}\left(m^{\prime}\right) \\
& -\phi=\operatorname{secret}(m) \text {. } \\
& (\operatorname{tr}, \operatorname{th}, \sigma, \vartheta) \not \models \operatorname{secret}(m) \\
& \Leftrightarrow I K(t r) \sigma, I K_{0} \vdash_{E} m \sigma \\
& \Rightarrow \operatorname{rem}_{T}(I K(t r)) \sigma, I K_{0} \vdash_{E} m \sigma \\
& \Leftrightarrow \operatorname{rem}_{T}(I K(t r)) \sigma, I K_{0} \vdash_{E} \operatorname{rem}_{T}(m) \sigma \\
& \text { by Proposition } 9 \\
& \Rightarrow I K\left(\mathrm{rem}_{T}(\mathrm{tr})\right) \sigma, I K_{0}^{\prime} \vdash_{E} \operatorname{rem}_{T}(m) \sigma \\
& \Leftrightarrow\left(\operatorname{rem}_{T}(\mathrm{tr}), \operatorname{rem}_{T}(t h), \sigma, \vartheta\right) \not \models \operatorname{secret}\left(\operatorname{rem}_{T}(m)\right)
\end{aligned}
$$

This completes the proof of the theorem. 


\section{G.3 Atom removal abstraction}

Atom removal abstractions allow us to eliminate atoms in clear. This cannot be done by typed or variable removal abstractions. Before we prove deducibility preservation for our atom removal abstractions, we introduce some auxiliary definitions. For any term $t$, we use atoms $(t)$ and fresh $(t)$ to denote the set of atoms and fresh values occurring in $t$, respectively. Recall that the attacker has a countably infinite choice of nonces $n_{i}^{\bullet} \in \mathcal{F}^{\bullet}$ for each type $\beta_{n}$. Given a set of atoms $A t \subseteq \mathcal{A} \cup \mathcal{C} \cup \mathcal{F}$ and a set of terms $T$, we define $\operatorname{imap}(A t, T)$ as the set of injective maps $\rho: \operatorname{tID}(A t, T) \rightarrow \mathbb{N}$ where $\operatorname{tID}(A t, T)=\left\{i \in T I D \mid f^{i} \in\right.$ $\left.\operatorname{fresh}\left(A t^{\sharp}\right) \cap \operatorname{split}(T)\right\}$. We extend $\rho$ to atoms and variables as follows.

- $\rho\left(n^{i}\right)=n_{\rho(i)}^{\bullet}$ for all $n^{i} \in \operatorname{fresh}\left(A t^{\sharp}\right) \cap \operatorname{split}(T)$,

- $\rho(u)=u$ for all remaining atomic messages $u \in \mathcal{A} \cup \mathcal{C} \cup \mathcal{V}^{b} \cup \mathcal{F}^{b} \cup \mathcal{F}^{\bullet}$.

We extend $\rho$ homomorphically to all terms. For the sake of uniformity, we treat $\rho$ as a substitution. For terms $t$ and $u$, we use $[t / u]$ to denote the replacement of $u$ by $t$.

Lemma 37. Let $t, u$ be terms, $a$ be an atom, and $\sigma$ be a substitution such that

(i) $\operatorname{vars}(t) \cap \operatorname{dom}(\sigma)=\emptyset$,

(ii) $a \notin \operatorname{vars}(u)$.

Then $(u \sigma)[t / a]=(u[t / a])(\sigma[t / a])$.

Proof. We prove this lemma by induction on $u$.

- If $u$ is an atom then $(u \sigma)[t / a]=u[t / a]$. Moreover, by (i), it follows that $(u[t / a])(\sigma[t / a])=u[t / a]$. Thus the lemma holds for this case.

- If $u$ is a variable then by (ii), we have $u[t / a]=u$. Therefore, we have $(u \sigma)[t / a]=u(\sigma[t / a])=(u[t / a])(\sigma[t / a])$.

- If $u=g\left(u_{1}, \ldots, u_{n}\right)$ for $g \in \Sigma^{n}, n \geq 1$, then we have

$$
\begin{array}{rlrl}
(u \sigma)[t / a] & =g\left(\left(u_{1} \sigma\right)[t / a], \ldots,\left(u_{n} \sigma\right)[t / a]\right) & & \text { since } a \text { is an atom } \\
& =g\left(\left(u_{1}[t / a]\right)(\sigma[t / a]), \ldots,\left(u_{n}[t / a]\right)(\sigma[t / a])\right) & \text { by IH } \\
& =\left(g\left(u_{1}, \ldots, u_{n}\right)[t / a]\right)(\sigma[t / a]) & & \text { since } a \text { is an atom } \\
& =(u[t / a])(\sigma[t / a]) & &
\end{array}
$$

This completes the proof of the lemma.

Next, we prove a deducibility lemma for atom removal abstractions.

Lemma 38. Let $T$ be set of normal-form terms, $t$ a normal-form term, At a set of atoms such that

(i) $A t^{\sharp}$ is clear in $T \cup\{t\}$, and

(ii) $A t^{\sharp} \cap \operatorname{fresh}\left(I K_{0}\right)=\emptyset$.

Let $\sigma$ be a substitution such that $T \sigma, I K_{0} \vdash_{E} t \sigma$. Then, for all $\rho \in \operatorname{imap}(A t, T \cup$ $\{t\})$, we have

$$
\operatorname{rem}_{A t}(T)(\rho \circ \sigma), I K_{0} \vdash_{E} \operatorname{rem}_{A t}(t)(\rho \circ \sigma) .
$$


Proof. We start by observing that under assumption (i) we have

$$
\operatorname{rem}_{A t}(u), \mathcal{F}^{\bullet} \vdash_{E} u \rho \text { and } u \rho \vdash_{E} \operatorname{rem}_{A t}(u)
$$

for all terms $u \in T \cup\{t\}$ and $\rho \in \operatorname{imap}(A t, T \cup\{t\})$.

Suppose $T \sigma, I K_{0} \vdash_{E} t \sigma$. We show that $\operatorname{rem}_{A t}(T) \theta, I K_{0} \vdash_{E} r e m_{A t}(t) \theta$ where $\theta=\rho \circ \sigma$. Together with $\mathcal{F}^{\bullet} \theta=\mathcal{F}^{\bullet}$ and $\mathcal{F}^{\bullet} \subseteq I K_{0}$, we can use Lemma 26 to derive the following from (36):

$$
\operatorname{rem}_{A t}(T) \theta, I K_{0} \vdash_{E}(T \rho) \theta \text { and }(t \rho) \theta \vdash_{E} \operatorname{rem}_{A t}(t) \theta
$$

Since $I K_{0} \rho=I K_{0}$ by assumption (ii), we can also apply Lemma 26 to the assumption $T \sigma, I K_{0} \vdash_{E} t \sigma$ and deduce $(T \sigma) \rho, I K_{0} \vdash_{E}(t \sigma) \rho$. Next, we use Lemma 37 to deduce $(T \rho) \theta, I K_{0} \vdash_{E}(t \rho) \theta$. Combining this with (37) above yields the desired result $\operatorname{rem}_{A t}(T) \theta, I K_{0} \vdash_{E} \operatorname{rem}_{A t}(t) \theta$.

Theorem 9 (Reachability preservation for atom removal abstractions). Let $P$ be a protocol and $A t \subseteq$ atoms $\left(\mathcal{M}_{P}\right)$ a set of atoms such that

(i) At is clear in $\mathcal{M}_{P}$, and

(ii) $A t^{\sharp} \cap \operatorname{fresh}\left(I K_{0}\right)=\emptyset$.

Let $(\operatorname{tr}, t h, \sigma)$ be a reachable state of $P$ and $\rho \in \operatorname{imap}(A t, \operatorname{term}(t r))$. Then the state $\left(\operatorname{rem}_{A t}(t r), \operatorname{rem}_{A t}(t h), \rho \circ \sigma\right)$ is a reachable state of rem $\mathrm{ret}_{A t}(P)$.

Proof. Let $\theta=\rho \circ \sigma$. It is clear that $\theta$ is well-typed. We now prove the first conclusion by induction on the number $n$ of transitions leading to a state $(t r, t h, \sigma)$. For the empty trace $(n=0)$, the theorem holds trivially. For the inductive case $(n=k+1)$, assume that $\left(t r^{\prime}, t h^{\prime}, \sigma\right)$ is reachable in $k$ steps and there is a transition $\left(t r^{\prime}, t h^{\prime}, \sigma\right) \rightarrow(t r, t h, \sigma)$. By induction hypothesis, $\left(r e m_{A t}\left(t r^{\prime}\right), r e m_{A t}\left(t h^{\prime}\right), \theta\right)$ is reachable in $\operatorname{rem}_{A t}(P)$.

There are two cases according to the rule $r$ that has been applied in the step $k+1$ in $P$. The case of the send rule is easy. We consider the case of receive rule. The rule's premises require that there are $i \in T I D, R \in \operatorname{dom}(P)$, and a suffix $t l$ of the role $P(R)^{\# i}$ such that

$$
t h^{\prime}(i)=(R, \operatorname{rcv}(t) \cdot t l) \quad \text { and } \quad I K\left(t r^{\prime}\right) \sigma, I K_{0} \vdash t \sigma
$$

The rule's conclusion implies that $t r=t r^{\prime} \cdot(i, \operatorname{rcv}(t))$ and $t h=t h^{\prime}[i \mapsto t l]$. In order to apply the $R E C V$ rule in the state $\left(r e m_{A t}\left(t r^{\prime}\right), r e m_{A t}\left(t h^{\prime}\right), \theta\right)$, we must show the following two premises

$$
\begin{aligned}
& r e m_{A t}\left(t h^{\prime}\right)(i)=\left(R, \operatorname{rcv}\left(r e m_{A t}(t)\right) \cdot r e m_{A t}(t l)\right), \text { and } \\
& \left.I K\left(r e m_{A t}\left(t r^{\prime}\right)\right)\right) \theta, I K_{0}^{\prime} \vdash_{E} r e m_{A t}(t) \theta .
\end{aligned}
$$

Clearly, the first premise is satisfied by application of $r e m_{A t}$ to $t h^{\prime}$. We now show the second one. Using Lemma 38, we deduce from (38) that

$$
\operatorname{rem}_{A t}\left(I K\left(t r^{\prime}\right)\right) \theta, I K_{0} \vdash_{E} r e m_{A t}(t) \theta
$$


Since $\operatorname{rem}_{A t}\left(I K\left(t r^{\prime}\right)\right)=I K\left(r e m_{A t}\left(t r^{\prime}\right)\right)$, we obtain

$$
I K\left(\operatorname{rem}_{A t}\left(t r^{\prime}\right)\right) \theta, I K_{0} \vdash_{E} \operatorname{rem}_{A t}(t) \theta .
$$

Moreover, the successor state in the conclusion of the $R E C V$ rule is

$$
\left(r e m_{A t}\left(t r^{\prime}\right) \cdot\left(i, \operatorname{rcv}\left(r e m_{A t}(t)\right)\right), \operatorname{rem}_{A t}\left(t h^{\prime}\right)\left[i \mapsto\left(R, r e m_{A t}(t l)\right)\right], \sigma\right),
$$

which is identical to the state $\left(r e m_{A t}(t r), \operatorname{rem}_{A t}(t h), \sigma\right)$. This concludes the proof of the theorem.

Definition 16 (( $P$, rem $\left._{A t}\right)$-safe formulas $)$. Let $P$ be a protocol. We assume a set of atoms $A t \subseteq$ atoms $\left(\mathcal{M}_{P}\right)$. A formula $\phi \in \mathcal{L}_{P}$ is $\left(P\right.$, rem $\left._{A t}\right)$-safe if the following conditions holds:

(i) $A t^{\sharp} \cap \operatorname{fresh}\left(\operatorname{Sec}_{\phi} \cup \operatorname{EqTerm}{ }_{\phi}\right)=\emptyset$,

(ii) for all $e(t) \in E v t_{\phi}$, we have $\operatorname{rem}_{A t}(t) \neq$ nil, and

(iii) $\operatorname{rem}_{A t}(m)=\operatorname{rem}_{A t}\left(m^{\prime}\right)$ implies $m=m^{\prime}$ for all $s(m) \in E v t_{\phi}^{+}$and $s\left(m^{\prime}\right) \in$ $\operatorname{Evt}\left(\mathcal{M}_{P}\right)$.

We now show soundness for atom removal abstractions.

Theorem 10 (Soundness for atom removal abstractions). Let $P$ be a protocol, $A t \subseteq \operatorname{atoms}\left(\mathcal{M}_{P}\right)$ and $\phi \in \mathcal{L}_{P}$ a formula such that

(i) At is clear in $\mathcal{M}_{P}$,

(ii) $A t^{\sharp} \cap \operatorname{fresh}\left(I K_{0}\right)=\emptyset$, and

(iii) $\phi$ is $\left(P\right.$, rem $\left._{A t}\right)$-safe

Then, for all reachable states $(t r, t h, \sigma)$ of $P$, there is a ground substitution $\theta$ such that

1. the state $\left(\operatorname{rem}_{A t}(t r)\right.$, rem $\left._{A t}(t h), \theta\right)$ is reachable in rem $\operatorname{rem}_{A t}(P)$, and

2. $(\mathrm{tr}, \mathrm{th}, \sigma) \not \models \phi$ implies $\left(\mathrm{rem}_{A t}(\mathrm{tr}), \operatorname{rem}_{A t}(\mathrm{th}), \theta\right) \not \models \operatorname{rem}_{A t}(\phi)$.

Proof. Let $\rho \in \operatorname{imap}(A t, \operatorname{term}(t r))$ such that

$$
\rho \text { is injective on } \operatorname{fresh}(\operatorname{ran}(\sigma)) \text {. }
$$

Let $\theta=\rho \circ \sigma$. By Theorem 9, we have that $\left(r e m_{A t}(t r), r e m_{A t}(t h), \theta\right)$ is reachable in $\operatorname{rem}_{A t}(P)$ (and thus $\theta$ is well-typed). Hence, it remains to show that

$$
\forall \vartheta .(\operatorname{tr}, t h, \sigma, \vartheta) \not \models \phi \Rightarrow\left(r e m_{A t}(t r), \operatorname{rem}_{A t}(t h), \theta\right) \not \models r e m_{A t}(\phi) .
$$

We proceed by induction on the structure of $\phi$ and consider the following nontrivial cases.

$$
\begin{aligned}
& -\phi \equiv m=m^{\prime} \text { or } \phi \equiv \neg\left(m=m^{\prime}\right) \text {. } \\
& (t r, t h, \sigma, \vartheta) \vDash m=m^{\prime} \\
& \Leftrightarrow m \sigma={ }_{E} m^{\prime} \sigma \\
& \Leftrightarrow(m \sigma) \rho={ }_{E}\left(m^{\prime} \sigma\right) \rho \\
& \Leftrightarrow(m \rho) \theta={ }_{E}\left(m^{\prime} \rho\right) \theta \\
& \Leftrightarrow \operatorname{rem}_{A t}(m) \theta={ }_{E} \operatorname{rem}_{A t}\left(m^{\prime}\right) \theta \quad \text { (by Def 16(ii)) } \\
& \Leftrightarrow\left(\operatorname{rem}_{A t}(t r), \operatorname{rem}_{A t}(t h), \theta, \vartheta\right) \vDash \operatorname{rem}_{A t}(m)=\operatorname{rem} m_{A t}\left(m^{\prime}\right)
\end{aligned}
$$


$-\phi=\operatorname{secret}(m)$.

$$
\begin{aligned}
& (t r, t h, \sigma, \vartheta) \not \models \operatorname{secret}(m) \\
\Leftrightarrow & I K(\operatorname{tr}) \sigma, I K_{0} \vdash_{E} m \sigma \\
\Rightarrow & \operatorname{rem} A t(I K(\operatorname{tr})) \theta, I K_{0} \vdash_{E} \operatorname{rem}_{A t}(m) \theta \quad(\text { by Lemma 38, Def 16(i)) } \\
\Rightarrow & I K\left(r e m_{A t}(t r)\right) \theta, I K_{0} \vdash_{E} \operatorname{rem}_{A t}(m) \theta \\
\Leftrightarrow & \left(\operatorname{rem}_{A t}(t r), r e m_{A t}(t h), \theta, \vartheta\right) \not \models \operatorname{secret}\left(\operatorname{rem}_{A t}(m)\right)
\end{aligned}
$$

This completes the proof of the theorem. 


\section{H Experimental results}

\section{H.1 Scyther tool}

The Scyther tool is based on symbolic backwards search and supports verification of both a bounded and an unbounded number of threads. We have demonstrated our abstraction method on a variety of protocols, mostly from the IKE and ISO/IEC 9798 families. Our results with the Scyther tool (version 1.1.2) are summarized in Table 2. Our experiments show substantial performance gains. The abstractions enable Scyther to verify seven protocols (four from the ISO/IEC 9798, two from the IKE families, and the PANA-AKA protocol) for an unbounded number of threads. Remarkably, five of them were verified (at the most abstract levels) within 0.4 seconds whereas it fails (TO) or runs out of memory (ME) on the original protocols.

For the IKE protocols, we approximate the Diffie-Hellman equations using oracle roles in Scyther. This complicates the verification task and, as a consequence, the average performance gain appears to be smaller than with the ISO/IEC protocols or the PANA-AKA protocol. In particular, the unbounded verification of (abstractions of) the first six IKE protocols in Table 2 still results in a timeout. However, we are able to either significantly improve the bounds on the number of threads that can be covered for these protocols or, for IKEv2-eap and IKEv2-eap2, enable protocol verification for a bounded number of threads where it timed out before even for three threads.

Apart from the dramatic speedups we achieve in most cases, we also observe that for many protocols the verification time increases much slower than their originals. For the last seven protocols in the table, this time is almost constant whereas it can grow rapidly, e.g., for ISO/IEC 9798-3-6-1 and PANA-AKA. Moreover, our abstractions greatly reduce memory consumption. In particular, Scyther runs out of memory for ISO/IEC 9798-3-6-1 and ISO/IEC 9798-3-7-1 after 5 and 6 threads, respectively. However, abstraction enables it to run up to an unbounded number of threads.

Scyther find attacks on the most abstract models much faster it does on the originals. Concretely, it falsifies the most abstract model of the IKEv1-sig-m and IKEv1-sig-m-perlman protocols for an unbounded number of threads within 0.06 seconds, while finding the same attacks on the original protocols takes 0.44 and 109 seconds, respectively. 


\begin{tabular}{|c|c|c|c|c|c|c|c|c|c|c|c|}
\hline protocol/prop./\#threac & $\mathrm{No}$ & $S \mid A$ & $W$ & $N$ & 3 & 4 & 5 & 6 & 7 & 8 & $\infty$ \\
\hline \multirow{2}{*}{ IKEv1-pk2-a2 } & 1 & 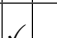 & & $x+2>$ & 44.01 & 302.86 & 1843.80 & 10999.80 & TO & TO & $\mathrm{TO}$ \\
\hline & 1 & $\left.\right|^{v}$ & & $v$ & 6.01 & 26.36 & 151.88 & 1014.63 & 6838.97 & TO & TO \\
\hline \multirow{2}{*}{ IKEv1-pk2-a } & 1 & 6 & & 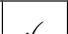 & 1103.63 & 27808.72 & TO & TO & TO & TO & $\mathrm{TO}$ \\
\hline & 1 & v & & $v$ & 133.65 & 3356.59 & TO & $\mathrm{TO}$ & TO & TO & $\mathrm{TO}$ \\
\hline \multirow{2}{*}{ IKEv1-pk-a2 } & 1 & I & & 4 & 10.95 & 61.47 & 125.25 & 237.76 & 409.35 & 744.75 & TO \\
\hline & 1 & $a^{v}$ & & $\checkmark$ & 0.84 & 1.79 & 2.43 & 3.63 & 6.01 & 9.61 & TO \\
\hline \multirow{2}{*}{ IKEv1-pk-a22 } & 1 & 16 & & & 18.48 & 82.93 & 249.55 & 554.09 & 1006.04 & 1734.85 & $\mathrm{TO}$ \\
\hline & 1 & $\left.\right|^{v}$ & & $\checkmark$ & 0.83 & 1.26 & 2.08 & 3.47 & 5.96 & 10.28 & $\mathrm{TO}$ \\
\hline \multirow{2}{*}{ IKEv2-eap } & 5 & $\checkmark$ & & $\checkmark$ & TO & $\mathrm{TO}$ & TO & TO & TO & TO & TO \\
\hline & 5 & $\left.\right|^{v}$ & & $\checkmark$ & 78.35 & 798.44 & 4212.71 & 20911.20 & TO & TO & $\mathrm{TO}$ \\
\hline \multirow{2}{*}{ IKEv2-eap2 } & & & & & TO & TO & TO & TO & TO & TO & TO \\
\hline & 5 & $\checkmark$ & & $\checkmark$ & 70.18 & 690.26 & 4169.87 & 20071.45 & TO & TO & $\mathrm{TO}$ \\
\hline \multirow{2}{*}{ IKEv2-mac } & 5 & & & & 1.85 & 4.91 & 6.72 & 8.07 & 8.42 & 8.49 & 8.70 \\
\hline & 5 & $\left.\right|^{v}$ & & $\checkmark$ & 0.62 & 1.77 & 1.83 & 1.73 & 1.73 & 1.80 & 1.74 \\
\hline \multirow{2}{*}{ IKEv2-mac2 } & 5 & & & 6 & 2.16 & 4.09 & 6.43 & 9.41 & 8.16 & 8.44 & 8.69 \\
\hline & 3 & $\left.\right|^{v}$ & & $v$ & 0.81 & 1.60 & 1.73 & 1.75 & 1.73 & 1.74 & 1.73 \\
\hline \multirow{2}{*}{ IKEv2-mactosig } & 4 & & & 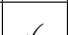 & 11.65 & 141.37 & 1075.46 & 7440.81 & TO & TO & TO \\
\hline & 4 & $\left.\right|^{v}$ & & $\checkmark$ & 2.89 & 12.38 & 24.54 & 38.68 & 53.36 & 65.07 & 77.68 \\
\hline \multirow{2}{*}{ IKEv2-mactosig2 } & 4 & & & 4 & 11.71 & 133.20 & 1064.30 & 7229.13 & TO & TO & TO \\
\hline & 4 & $\left.\right|^{v}$ & & $v$ & 2.85 & 11.81 & 24.14 & 38.22 & 53.25 & 64.51 & 77.03 \\
\hline \multirow{2}{*}{ IKEv2-sigtomac } & & & & & 6.15 & 33.19 & 65.05 & 115.34 & 204.93 & 206.45 & 237.34 \\
\hline & 5 & $\checkmark$ & & $\checkmark$ & 3.59 & 12.72 & 28.44 & 44.44 & 55.11 & 66.97 & 67.15 \\
\hline \multirow{2}{*}{ IKEv1-pk-m } & 2 & & & $x$ & 48.62 & 269.92 & 507.40 & 869.23 & 16254.80 & TO & TO \\
\hline & 2 & & & $x$ & 0.04 & 0.05 & 0.05 & 0.05 & 0.05 & 0.05 & $\mathrm{TO}$ \\
\hline \multirow{2}{*}{ IKEv1-pk-m2 } & & & & & 18.26 & 274.87 & 4438.72 & TO & TO & TO & TO \\
\hline & 2 & & & $v / x$ & 1.48 & 7.79 & 32.75 & 110.32 & 339.93 & 963.08 & $\mathrm{TO}$ \\
\hline \multirow{2}{*}{ IKEv1-sig-m } & 2 & & & & 0.34 & 0.45 & 0.45 & 0.45 & 0.45 & 0.46 & 0.44 \\
\hline & 2 & & & $x$ & 0.05 & 0.05 & 0.05 & 0.06 & 0.05 & 0.05 & 0.06 \\
\hline \multirow{2}{*}{ IKEv1-sig-m-perlman } & 2 & & & $y$ & 2.86 & 13.99 & 40.78 & 67.83 & 72.08 & 72.15 & 109.03 \\
\hline & 2 & & & $x$ & 0.05 & 0.05 & 0.05 & 0.05 & 0.05 & 0.05 & 0.05 \\
\hline \multirow{2}{*}{ ISO/IEC 9798-2-5 } & 1 & & & & 0.78 & 8.96 & 73.87 & 564.67 & 4214.22 & TO & $\mathrm{TO}$ \\
\hline & 1 & | & & & 0.07 & 0.11 & 0.12 & 0.11 & 0.11 & 0.11 & 0.11 \\
\hline \multirow{2}{*}{ ISO/IEC 9798-2-6 } & & & & & 0.57 & 3.74 & 18.42 & 67.01 & 196.30 & 488.04 & 21278.58 \\
\hline & 1 & $\checkmark$ & & & 0.05 & 0.04 & 0.05 & 0.05 & 0.05 & 0.05 & 0.05 \\
\hline \multirow{2}{*}{ ISO/IEC 9798-3-6-1 } & & & & & 43.08 & 802.95 & 8903.70 & $\mathrm{ME}$ & $\mathrm{ME}$ & $\mathrm{ME}$ & $\mathrm{ME}$ \\
\hline & 2 & $\checkmark$ & & $\checkmark$ & 0.13 & 0.18 & 0.19 & 0.19 & 0.19 & 0.19 & 0.19 \\
\hline \multirow{2}{*}{ ISO/IEC 9798-3-6-2 } & 1 & & & 保 & 2.74 & 8.67 & 19.56 & 33.91 & 52.51 & 69.48 & 90.04 \\
\hline & 1 & $\checkmark$ & & $\checkmark$ & 0.12 & 0.15 & 0.15 & 0.15 & 0.15 & 0.15 & 0.15 \\
\hline \multirow{2}{*}{ ISO/IEC 9798-3-7-1 } & & & & & 40.43 & 740.47 & 7483.36 & 16631.42 & ME & ME & $\mathrm{ME}$ \\
\hline & 2 & $\checkmark$ & & $\checkmark$ & 0.13 & 0.18 & 0.19 & 0.19 & 0.19 & 0.19 & 0.19 \\
\hline \multirow{2}{*}{ ISO/IEC 9798-3-7-2 } & 1 & 6 & & 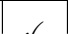 & 2.38 & 7.71 & 16.68 & 26.99 & 35.06 & 49.49 & TO \\
\hline & 1 & $\checkmark$ & & $\checkmark$ & 0.22 & 0.32 & 0.33 & 0.33 & 0.33 & 0.33 & 0.33 \\
\hline PANA-AKA & 5 & 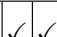 & & $f(x)+$ & 5769.53 & TO & TO & TO & TO & TO & $\mathrm{TO}$ \\
\hline PANA-AKA & 0 & & & $v$ & 0.10 & 0.10 & 0.10 & 0.10 & 0.10 & 0.10 & 0.10 \\
\hline
\end{tabular}

Table 2. Experimental results. The time is in seconds. No: Number of abstractions. Properties of interest are Secrecy, Aliveness, Weak agreement, and Non-injective agreement. 


\section{H.2 Avantssar tools}

The AVANTSSAR platform is an integrated toolset for the formal specification and Automated VAlidatioN of Trust and Security of Service-oriented ARchitectures. It provides three validation back-ends (CL-Atse, OFMC, and SATMC) which share the input languages for specifying protocols. The validators are based on two different techniques. SATMC reduces protocol insecurity problems to the satisfiability of propositional formulas which can then be checked by modern SAT solvers. CL-Atse and OFMC both use constraint solving techniques to search for attacks. However, they use different optimization strategies to reduce the search space. All these tools can verify protocols only for a bounded number of threads.

We have experimented with CL-Atse (version 2.5-21), OFMC (version 2013b), and SATMC (version 3.4) on several protocols from IKE and ISO/IEC 9798 families. Moreover, we have performed experiments on variants of the TLS and basic Kerberos protocols. For TLS, we distinguish two instances according to different security properties of interest. So far, we have not modelled IKE protocols for SATMC, as this requires substantial effort to encode oracles for Diffie-Hellman equations. We therefore defer extended experiments with SATMC to future work.

In our experiments, we measure the verification time for different numbers of sessions. Note that a session in CL-Atse, SATMC, and OFMC differs from a thread in Scyther. CL-Atse and SATMC specify a session as an instantiation of all protocol roles, not just a single role. For instance, a session of a protocol with three different roles results in three role instances (or three threads in Scyther) where a concrete agent is assigned to each role. In contrast, OFMC works with symbolic sessions where the agents executing the roles are not concretely specified but kept as variables.

For the AVANTSSAR tools, our experimental results generally exhibit smaller speedups than for Scyther. There is also a considerable variance between the different tools.

CL-Atse (Table 3) CL-Atse shows minor performance gains for the two IKEv1 protocols (pk2-a and pk-a2). However, abstraction enables the verifications of first three IKEv2 protocols (eap, eap2, and mac) for four sessions in less than 2 hours and dramatically speeds up the verification of three sessions of the eap and eap2 variants by factors greater than 690 and 900, respectively. For the last two IKEv2 protocols, the performance gains are still substantial: for four sessions we achieve a speedup factor of 7 for IKEv2-mactosig and of 107 for IKEv2-sigtomac. The best result in the ISO/IEC family is achieved for the ISO/IEC 9798-2-5 protocol where we can turn a timeout for 10 sessions into a time less than 0.2 seconds. The speedup for the $2-5$ variant is less impressive and for the two 3-7 variants, we even observe an increase in verification time as we do for the basic Kerberos protocol. For TLS, the verification time of secrecy up to five sessions drops from 260 minutes to 6 minutes (factor 42), whereas that of authentication is sped up by a factor of 1.5 for four sessions.

OFMC (Table 4) Surprisingly, the experimental results for OFMC are almost dual to those for CL-Atse. In particular, for the two IKEv1 protocols, OFMC 
loses performance on the abstracted protocols compared to the originals. Nevertheless, the abstractions save a lot of effort for the remaining protocols. We are able to increase the number of tractable sessions for 8 protocols: for 2 out of 7 from the IKE family, 5 out of 6 from the ISO/IEC 9798 family, and for the basic Kerberos protocol. For TLS, the verification of authentication is 1.7 times faster (up to 3 sessions). For secrecy, the tool achieves a 20 -fold speedup (up to 4 sessions). As a typical case, OFMC verifies an abstraction of ISO/IEC 9798-2-5 for 5 sessions within less than 4 seconds whereas it times out on the original for more than 2 sessions.

SATMC (Table 5) The abstractions enable the verification of the Kerberos and TLS protocols for 5 and even 10 sessions. In particular, the tool takes less than 21 seconds to verify the abstracted TLS protocol for 10 sessions whereas it times out for 5 sessions of the original protocol. On the negative side, SATMC loses performance for the protocols in the ISO/IEC family.

Apart from positive results, our experiments also provide an evidence that protocol abstractions are not always helpful. This is typically the case when an abstraction removes sensitive information. In particular, the performance degradation for the AVANTSSAR tools can possibly be attributed to an interference with the highly refined optimization techniques used in these tools. More precisely, an abstraction may get rid of data that is crucial to eliminate redundancies (for CL-Atse) or to limit the number of branching nodes in the symbolic search tree (for OFMC). As a result, the search space becomes larger in the abstracted protocols than in the originals. However, the influence of abstraction on the SATMC's performance is not clear. A further investigation is therefore desirable.

\section{H.3 ProVerif tool}

ProVerif is an automated cryptographic protocol verifier in the standard DolevYao model. It supports user-defined equational theories to model algebraic properties of cryptographic primitives. In constrast with Scyther, it uses approximations, e.g., translating protocol models in the applied pi calculus to a set of Horn clauses, to handle an unbounded number of sessions. These approximations are sound with respect to attacks, i.e., if the tool finds no attacks then the protocol is indeed secure.

We have validated our abstractions for ProVerif (version 1.88) on six protocols from the IKE and ISO/IEC 9798 families (see Table 6). For all these protocols, we observe good speedups. In particular, for the IKEv1-pk-a2 and the IKEv2-eap, the speedup factors are 6 and 5, respectively. The performance gains for the ISO/IEC 9798 protocols are less obvious than for the IKE ones. Concretely, the tool is roughly 1.5 times faster for these protocols. 


\begin{tabular}{|c|c|c|c|c|c|c|c|}
\hline protocol/prop./\#sessions & $S$ & $A \mid W$ & $N$ & 3 & 4 & 5 & 10 \\
\hline \multirow{2}{*}{ IKEv1-pk2-a } & \multirow[b]{2}{*}{$\checkmark$} & & \multirow[b]{2}{*}{$\checkmark$} & 0.06 & 0.11 & 0.53 & $\mathrm{TO}$ \\
\hline & & & & 0.05 & 0.08 & 0.32 & $\mathrm{TO}$ \\
\hline \multirow{2}{*}{ IKEv1-pk-a2 } & \multirow{2}{*}{$\checkmark$} & & \multirow{2}{*}{$\checkmark$} & 0.05 & 0.09 & 1.79 & \\
\hline & & & & 0.05 & 0.07 & 1.17 & $\mathrm{TO}$ \\
\hline \multirow{2}{*}{ IKEv2-eap } & \multirow[b]{2}{*}{$\checkmark$} & & \multirow[b]{2}{*}{$\checkmark$} & 625.75 & TO & TO & \\
\hline & & & & 23.17 & $\mathrm{TO}$ & TO & 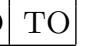 \\
\hline \multirow{2}{*}{ IKEv2-eap2 } & \multirow[b]{2}{*}{$\checkmark$} & & \multirow[b]{2}{*}{$\checkmark$} & 1248.57 & $\mathrm{TO}$ & TO & \\
\hline & & & & 37.93 & $\mathrm{TO}$ & $\mathrm{TO}$ & 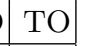 \\
\hline \multirow{2}{*}{ IKEv2-mac } & \multirow[b]{2}{*}{$\checkmark$} & & \multirow[b]{2}{*}{$\checkmark$} & 2.78 & TO & TO & \\
\hline & & & & 0.89 & 5830.38 & $\mathrm{TO}$ & TC \\
\hline \multirow{2}{*}{ IKEv2-mactosig } & \multirow{2}{*}{$\checkmark$} & & \multirow{2}{*}{$\checkmark$} & 0.24 & 1056.31 & $\mathrm{TO}$ & $\mathrm{TC}$ \\
\hline & & & & 0.12 & 149.19 & $\mathrm{TO}$ & 1 \\
\hline \multirow{2}{*}{ IKEv2-sigtomac } & \multirow{2}{*}{$\checkmark$} & & \multirow{2}{*}{$\checkmark$} & 2.52 & 16710.31 & TO & $\mathrm{T}$ \\
\hline & & & & 0.10 & 155.63 & $\mathrm{TO}$ & $1 \mathrm{~T}$ \\
\hline \multirow{2}{*}{ ISO/IEC 9798-2-5 } & \multirow[b]{2}{*}{$\checkmark$} & & & 20.05 & $\mathrm{TO}$ & TO & $\mathrm{TO}$ \\
\hline & & & & 0.52 & 4064.93 & 0.18 & 0.17 \\
\hline \multirow{2}{*}{ ISO/IEC 9798-2-6 } & \multirow[b]{2}{*}{$\checkmark$} & & & 1639.32 & $\mathrm{TO}$ & TO & $\mathrm{T}$ \\
\hline & & & & 703.55 & $\mathrm{TO}$ & $\mathrm{TO}$ & 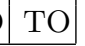 \\
\hline \multirow{2}{*}{ ISO/IEC 9798-3-7-1 } & & 1 & \multirow{2}{*}{$\checkmark$} & 1.21 & 4495.43 & TO & TC \\
\hline & & $\checkmark$ & & 1973.78 & $\mathrm{TO}$ & TO & ) \\
\hline \multirow{2}{*}{ ISO/IEC 9798-3-7-2 } & & $\checkmark$ & \multirow{2}{*}{$\checkmark$} & 29.95 & TO & TO & $\mathrm{TC}$ \\
\hline & & $\checkmark$ & & $\mathrm{TO}$ & TO & $\mathrm{TO}$ & 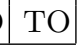 \\
\hline & & & & 0.30 & 0.29 & 22473.21 & $\mathrm{TC}$ \\
\hline Kerb- & & $\checkmark$ & & 0.18 & 0.18 & $\mathrm{TO}$ & \\
\hline & & & & 0.10 & 60.02 & TO & $\mathrm{TO}$ \\
\hline TLS- & & & $\checkmark$ & 0.08 & 39.42 & $\mathrm{TO}$ & $\mathrm{TC}$ \\
\hline TL & & & $\sqrt{ }$ & 0.07 & 8.63 & 15551.63 & $\mathrm{TC}$ \\
\hline $1 \mathrm{LS}$ & & & $\checkmark$ & 0.05 & 0.51 & 369.57 & \\
\hline
\end{tabular}

Table 3. Experimental verification results for CL-Atse. The time is in seconds. 


\begin{tabular}{|c|c|c|c|c|c|c|c|c|}
\hline protocol/prop./\#ses & $S$ & $A$ & $W$ & $N$ & 2 & 3 & 4 & 5 \\
\hline \multirow{2}{*}{ IKEv1-pk2-a } & \multirow{2}{*}{$\checkmark$} & & & \multirow[b]{2}{*}{$\checkmark$} & 36.28 & 27745.29 & TO & TO \\
\hline & & & & & 59.10 & TO & TO & TO \\
\hline \multirow{2}{*}{ IKEv1-pk-a2 } & \multirow{2}{*}{$\checkmark$} & & & \multirow[b]{2}{*}{$\checkmark$} & 4.28 & 849.46 & TO & $\mathrm{TO}$ \\
\hline & & & & & 12.09 & 9192.14 & TO & TO \\
\hline \multirow{2}{*}{ IKEv2-eap } & \multirow{2}{*}{$\checkmark$} & & & \multirow[b]{2}{*}{$\checkmark$} & 8920.57 & $\mathrm{TO}$ & TO & TO \\
\hline & & & & & 10.07 & 8942.94 & $\mathrm{TO}$ & TO \\
\hline \multirow{2}{*}{ IKEv2-eap2 } & \multirow{2}{*}{$\checkmark$} & & & \multirow{2}{*}{$\checkmark$} & 5407.00 & $\mathrm{TO}$ & TO & $\mathrm{TO}$ \\
\hline & & & & & 46.14 & TO & TO & $\mathrm{TO}$ \\
\hline \multirow{2}{*}{ IKEv2-mac } & \multirow{2}{*}{$\checkmark$} & & & \multirow{2}{*}{$\checkmark$} & 18.59 & 22547.87 & TO & $\mathrm{TO}$ \\
\hline & & & & & 11.19 & 16139.98 & TO & $\mathrm{TO}$ \\
\hline \multirow{2}{*}{ IKEv2-mactosig } & \multirow{2}{*}{$\checkmark$} & & & \multirow{2}{*}{$\checkmark$} & 22.08 & 15561.69 & TO & $\mathrm{TO}$ \\
\hline & & & & & 9.27 & 10605.58 & 11782.39 & $\mathrm{TO}$ \\
\hline \multirow{2}{*}{ IKEv2-sigtomac } & \multirow{2}{*}{$\checkmark$} & & & \multirow{2}{*}{$\checkmark$} & 18.58 & 13617.91 & TO & $\mathrm{TO}$ \\
\hline & & & & & 12.36 & 12408.54 & TO & $\mathrm{TO}$ \\
\hline \multirow{2}{*}{ ISO/IEC 9798-2-5 } & \multirow{2}{*}{$\checkmark$} & & & & 805.64 & $\mathrm{TO}$ & $\mathrm{TO}$ & $\mathrm{TO}$ \\
\hline & & & & & 3.61 & 3.43 & 3.85 & 3.59 \\
\hline \multirow{2}{*}{ ISO/IEC 9798-2-6 } & \multirow{2}{*}{$\checkmark$} & & & & 7232.17 & $\mathrm{TO}$ & $\mathrm{TO}$ & $\mathrm{TO}$ \\
\hline & & & & & 144.06 & TO & $\mathrm{TO}$ & $\mathrm{TO}$ \\
\hline \multirow{2}{*}{ ISO/IEC 9798-3-6-1 } & & & & \multirow{2}{*}{$\checkmark$} & 17941.80 & $\overline{\mathrm{TO}}$ & TO & $\mathrm{TO}$ \\
\hline & & $\checkmark$ & & & 27.92 & 18019.32 & TO & TO \\
\hline ISO/IFC $0708-3-6$ & & 6 & & 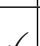 & $\mathrm{TO}$ & $\mathrm{TO}$ & TO & $\mathrm{TO}$ \\
\hline ISU/1EC $9798-3-6-2$ & & $\checkmark$ & & $\checkmark$ & 12.97 & 3673.20 & $\mathrm{TO}$ & $\mathrm{TO}$ \\
\hline & & & & & TO & $\mathrm{TO}$ & TO & $\mathrm{TO}$ \\
\hline 1SU/1EC 9798-3-7-1 & & $\checkmark$ & & $\checkmark$ & 50.52 & TO & TO & $\mathrm{TO}$ \\
\hline ISO/IFC 0708278 & & & & & TO & $\mathrm{TO}$ & TO & $\mathrm{TO}$ \\
\hline ISU/1EC 9798-3-7-2 & & $\checkmark$ & & $\checkmark$ & 11.61 & 4010.64 & $\mathrm{TO}$ & $\mathrm{TO}$ \\
\hline & & & & & 20.63 & $\overline{\mathrm{TO}}$ & $\overline{\mathrm{TO}}$ & TO \\
\hline Kerb-basic & & & $\checkmark$ & & 8.07 & 28699.72 & TO & $\mathrm{TO}$ \\
\hline TLS-auth & & & & $\checkmark$ & 9.12 & 6002.38 & TO & $\mathrm{TO}$ \\
\hline & & & & $\checkmark$ & 8.88 & 3549.25 & TO & $\mathrm{TO}$ \\
\hline TLS-sec & & & & $\checkmark$ & 0.27 & 13.62 & 1304.21 & TO \\
\hline & & & & 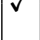 & 0.15 & 1.97 & 59.87 & $\mathrm{TO}$ \\
\hline
\end{tabular}

Table 4. Experimental verification results for OFMC. The time is in seconds. 


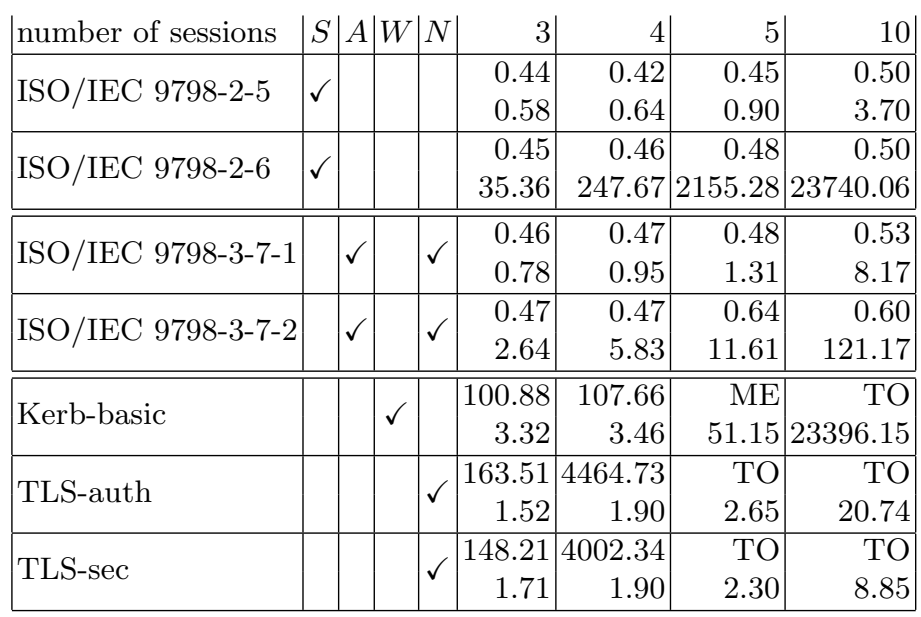

Table 5. Experimental verification results for SATMC. The time is in seconds.

\begin{tabular}{|c|c|c|c|c|}
\hline |protocol/prop./\#threads & $S$ & $A \mid W$ & $J$ & $\infty$ \\
\hline IKEv1-pk2-a & $\checkmark$ & & $\checkmark$ & 43.53 \\
\hline IKEv1-pk-a2 & $\star$ & & & $\begin{array}{r}.84 \\
0.3\end{array}$ \\
\hline IKEv2-eap & $\checkmark$ & & $\checkmark$ & $\begin{array}{r}22.27 \\
4.22\end{array}$ \\
\hline IKEv2-mactosig & $\checkmark$ & & $\checkmark$ & $\begin{array}{l}4.57 \\
0.91 \\
\end{array}$ \\
\hline ISO/IEC 9798-2-5 & $\checkmark$ & & & $\begin{array}{l}0.09 \\
0.06\end{array}$ \\
\hline ISO/IEC 9798-3-7-1 & & & $\checkmark$ & $\begin{array}{l}0.13 \\
0.08\end{array}$ \\
\hline
\end{tabular}

Table 6. Experimental verification results for ProVerif. The time is in seconds. The $\star$ presents ProVerif verifies the property for one role and cannot prove it for the other. 